ISSN 0280-5316

ISRN LUTFD2/TFRT--5853--SE

\title{
Probing control in B. Cicheniformis fermentations
}

Ola Johnsson

Department of Automatic Control

Lund University May 2010 



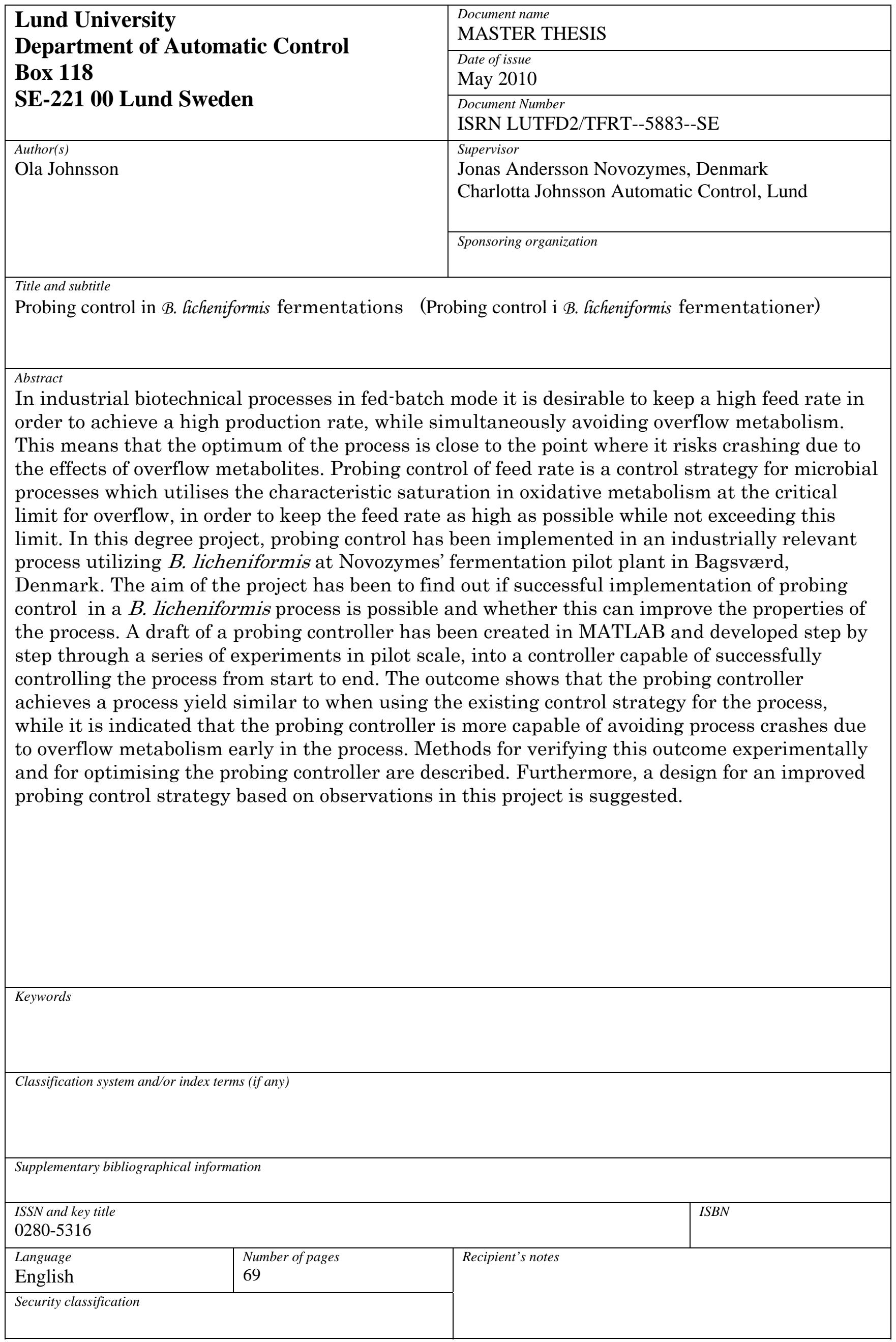

http://www.control.lth.se/publications/ 



\section{Contents}

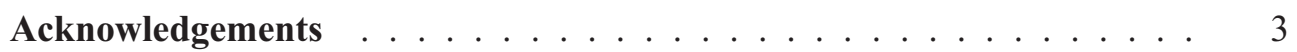

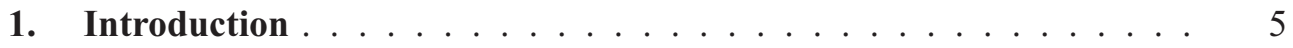

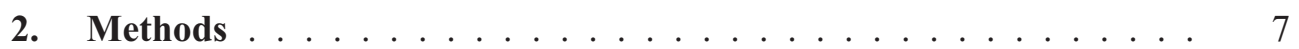

2.1 Work approach . . . . . . . . . . . . . . . 7

2.2 Layout of thesis . . . . . . . . . . . . . . . . . . . 7

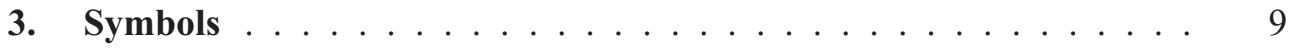

4. Background . . . . . . . . . . . . . . . . . . . . 10

4.1 Bioreactors and fed-batch processes . . . . . . . . . . . 10

4.2 Substrate metabolism in cells . . . . . . . . . . . . . . 11

4.3 Probing control of the feed rate in fed-batch fermentations . . . . . 13

4.4 Bacillus licheniformis . . . . . . . . . . . . . . . . . . . . . . 14

4.5 Novozymes . . . . . . . . . . . . . . . . . . . . . 14

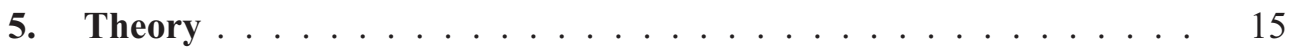

5.1 A model of microbial growth and production in fed-batch fermentations . . . . . . . . . . . . . . . . 15

5.2 Probing control . . . . . . . . . . . . . . . . . 19

6. The process and the experimental setup . . . . . . . . . . . . . 24

6.1 General information on the process . . . . . . . . . . . . 24

6.2 Practicalities of controller implementation . . . . . . . . . 26

6.3 Data sampling during experiments . . . . . . . . 26

7. The current control strategy . . . . . . . . . . . . . . . . . . 28

7.1 Reference experiment . . . . . . . . . . . . . . . . . . 29

8. Development experiments . . . . . . . . . . . . . . . . . 31

8.1 First and second development experiments . . . . . . . . . 32

8.2 Third development experiment . . . . . . . . . . . . . . . . 34

8.3 Fourth development experiment . . . . . . . . . . . . 36

8.4 Fifth, sixth and seventh development experiments . . . . . . . . 40

8.5 Eighth development experiment . . . . . . . . . . . . . . 44

8.6 Discussion of development experiments . . . . . . . . . . . 47

8.7 Features of the final controller . . . . . . . . . . . . 50

9. Evaluation experiment . . . . . . . . . . . . . . . . . 53

9.1 Discussion of evaluation experiment . . . . . . . . . . . 54

10. Future work . . . . . . . . . . . . . . . . . . . . . . . 59

10.1 Proof of improved process robustness $\ldots \ldots \ldots$

10.2 Improving control of the process . . . . . . . . . . . . . . 59

10.3 Improving the probing control strategy $\ldots \ldots \ldots \ldots$

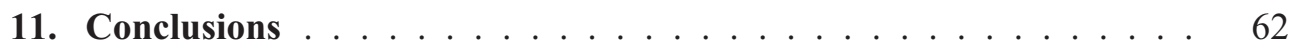

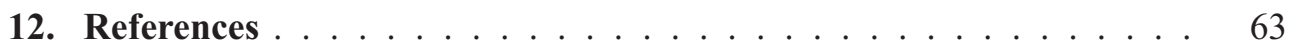

A. Fermentation summary $\ldots \ldots \ldots \ldots 65$

B. Sampling procedures $\ldots \ldots \ldots \ldots 6$ 


\section{Acknowledgements}

The thesis project presented in this report has been performed at Novozymes' fermentation pilot plant in Bagsværd, Denmark. It has been a great experience to make my master thesis at a major biotech company and I have enjoyed it much, both because I was given the opportunity to work within an area which I find very interesting and thanks to the people working at the fermentation pilot plant who have always been helpful and friendly. They all deserve a big thank you, but I would like to direct a special thanks to some of them.

First of all I would like to thank my supervisor Jonas Andersson who has been very helpful in everything from explaining how to plan fermentations to taking samples when I could not be present to do so myself, as well as helping me get to know Novozymes and the people at the fermentation pilot plant. I think it would not be possible to ask any more from a supervisor. Both he and Stuart Stocks have given me some very valuable input on my work and taught me a lot regarding the practical issues surrounding it, which I am much grateful for. Henning Fugmann deserves a thanks for taking his time to reprogram DeltaV for my experiments and Martin Eriksen and Frank Jeske Lausen for helping me run my 201 fermentations.

I would also like to thank my examinator Charlotta Johnsson, who has not only been supportive and given me good advice, but whose course in process control also opened my eyes to this area in the first place. The professors Gunnar Lidén and Per Hagander have contributed with some of their knowledge within the fields of bioprocesses respectively automatic control, which I am very grateful for. Finally I would like to thank my friend Anna Hansson who helped me get into contact with Novozymes and thus made it possible for me to make this thesis project. 



\section{Introduction}

Genetically modified microorganisms are used to produce a wide range of products in industrial processes today. They can both be used to create compounds which would not be possible to produce industrially otherwise, such as insulin and human growth hormone, as well as for reducing the need for harmful chemicals and/or extreme temperatures in the processes, making them both more economical and less harmful to the environment, such as in the production of lactic acid and butanol. Genetically engineered microorganisms have only been used in industrial processes for about 30 years, but their usage has increased rapidly. As in all industry-related areas, developing new and more efficient processes is of interest here.

Developing and improving a bioprocess can be done in several ways. One approach is to find a new, more efficient microorganism which can be engineered to produce the desired product. Another method is to use genetic engineering to improve the traits of a currently used microorganism. The process also becomes more efficient if optimal conditions for the microorganism can be offered in the bioreactor. Other means of improving the process are also possible, which are not related to the bioreactor, such as improved downstream processing.

There is a multitude of parameters which need to be taken into account when designing the process. When looking at the bioreactor, which is where the microbial growth and product production takes place, some of the most important parameters are $\mathrm{pH}$, temperature and concentration of dissolved oxygen as well as which sources of carbon, nitrogen and trace elements are available and at what concentrations. If optimal conditions cannot be given, this will of course influence the outcome of the process in a negative way. With a robust microorganism or small deviations from the optimal conditions, the microorganism may simply work less efficiently leading to a lower yield of the process. Too large deviations may cause the process to crash, meaning that the microorganisms either die or for some other reason cease to produce the desired product entirely.

Process control in bioprocesses has the purpose of providing the optimal environment for the the microorganism during the entire fermentation process. Although the overall goal of the process is to achieve a high final product concentration, the objective of the process control system can vary throughout a process. Typically, a fast growth of biomass is desired at the start of a process, while a high productivity in regard to the desired product is the goal later in the process. To be successful in this, control theory must be combined with an understanding of the microorganism and how its metabolism changes under different conditions.

Several mechanisms can lead to a less than optimal bioprocess, such as substrate and product inhibition, starvation, overflow metabolism and oxygen limitation. Overflow metabolism is caused by a too high level of substrate in the medium, leading to the production of undesired by-products which will often harm the microorganism. Oxygen limitation means that little or no oxygen is present and will be consumed as fast as it is supplied, which typically occurs late in the process when the biomass is large enough that the maximum oxygen transfer rate of the bioreactor becomes limiting, requiring a decreased feed rate to avoid this effect. Good control of the feed rate is therefore a desirable trait in bioprocesses, to keep it as high as possible while avoiding overflow metabolism and oxygen limitation.

Probing control of feeding is a control strategy in which pulses are superimposed on a feed rate baseline and the response or lack thereof in the dissolved oxygen level is used to set the new feed rate baseline level, as a lack of response indicates that 
overflow metabolism is reached. Ideally, this allows the controller to find a feed rate just below the onset of overflow metabolism, thus giving a high feed and thereby higher microbial growth and production while avoiding the production of undesired byproducts. Since it was first described in 1999, several variants of it have been developed and it has been implemented in fermentations from lab-scale to industrial scale. Although probing control of feeding was first developed in Escherichia coli fermentations, it is a fairly general method and has been used in processes utilising for instance Vibrio cholerae and Saccharomyces cerevisiae.

The Danish company Novozymes uses industrial scale fed-batch fermentations to produce a variety of enzyme products, such as proteases. One of these protease processes which utilises the bacterium Bacillus licheniformis occasionally crashes, which is believed to be caused by harmful substances produced in overflow metabolism. A similar process can be run in pilot scale at Novozymes' fermentation pilot plant in Bagsværd, Denmark, to try out modifications to the process before implementing them in industrial scale. As the crashes are believed to be due to overflow metabolism, the process appears to be a suitable subject for probing control. The goal of the project is as follows:

The aim of this thesis project is to find out if it is possible to implement probing control of the feed rate in B. licheniformis fermentations at Novozymes' fermentation pilot plant, possibly making them more resistent to crashing while keeping a product yield similar to that when using the currently existing control strategy. 


\section{Methods}

\subsection{Work approach}

The basic methodology behind this thesis is one of problem solving, seeking to improve the characteristics of an industrial process through applying a previously known control strategy which has not been implemented in this process before. The approach has been one of experimental work and action research, in which the process was first observed to ensure its likeness to other processes where the relevant control strategy has been successfully applied previously, then development work was performed to successfully implement the technology.

To develop the probing control strategy in the context of B. licheniformis fedbatch fermentations, a controller was created in MATLAB and pilot-scale fermentations employing this controller were performed. The initial controller was based directly on the one described in (Åkesson 1999, paper V). The development work was an iterative process, where the outcome of each experiment led to development in the setup of the fermentations and in the controller, which were in turn evaluated in the next experiment. The aim of this procedure was to continuously improve the controller from a first draft to a working strategy which could successfully control the process from start to end without operator input. After the development work, the outcome was evaluated by making a comparison between the process when utilising the currently used control strategy and when utilising the strategy implemented in this project.

\subsection{Layout of thesis}

This thesis lies within the area of bioprocess control, which combines elements of biotechnology with automatic control. To provide an understanding of the issues addressed in this thesis project and the principles behind the work done here, the Background chapter contains general information regarding bioprocesses and the basic theory behind probing control. This chapter also presents the microorganism which is featured in this thesis as well as Novozymes, the company at which the work during the thesis project has been done.

The Theory part then goes deeper into describing the relevant process by providing a simple model for describing microbial growth and production. The purpose of this section is to give a more in-depth understanding of the assumptions on which the probing control strategy is based. The probing control strategy itself is then described in detail, with particular focus on the variant which has been the basis for the controller developed in this project.

The chapter titled The process and the experimental setup gives a background to the experimental work done in this project, by describing the specific process which the project has been focused on as well as outlining the practicalities surrounding the experiments.

As the aim of this project has been to implement a new control strategy which may possibly improve the properties of the process, the existing control strategy should also be studed. This is done in the chapter titled The current control strategy, which also describes a reference experiment performed using this strategy. 
After the process and its current controller have been outlined, the Development experiments chapter goes through the experiments performed in order to develop a probing controller capable of controlling the process and discusses these as well as other possible ways of development. The Evaluation experiment chapter then details the experiment performed to evaluate the process when controlled by the probing controller and compares the outcome to that of the reference experiment.

The Future work chapter sums up and discusses possible means of evaluating the outcome of this project further and improving the controller which has been developed as well as describing the apparent possibilites for an alternate probing control strategy based on observations made in this project. Finally, the Conclusions chapter provides a summary of the outcome of the whole project. 


\section{Symbols}

The parameters, symbols and units in table 3.1 are used throughout this report. $s p$ after a symbol indicates a setpoint while ${ }_{p v}$ indicates a measured process value.

\begin{tabular}{|c|c|c|}
\hline Parameter & Symbol & Unit \\
\hline \multicolumn{3}{|l|}{ Process parameters } \\
\hline \multirow[t]{2}{*}{ Volume of fermentation medium } & V & 1 (25 1 fermentations) \\
\hline & & $\mathrm{m}^{3}\left(2.5 \mathrm{~m}^{3}\right.$ fermentations $)$ \\
\hline Dissolved oxygen saturation & $D O$ & $\%$ \\
\hline \multirow{2}{*}{ Feed rate } & $F$ & $\mathrm{~g} / \mathrm{h}$ (25 1 fermentations $)$ \\
\hline & & $1 / \mathrm{h}\left(2.5 \mathrm{~m}^{3}\right.$ fermentations $)$ \\
\hline Agitator speed & $N$ & $\mathrm{rpm}$ \\
\hline Aeration rate & $Q_{\text {gas, in }}$ & $1 / \mathrm{min}$ \\
\hline Dissolved oxygen concentration & $\mathrm{O}$ & $\mathrm{g} / 1$ \\
\hline Biomass concentration & $X$ & $\mathrm{~g} / 1$ \\
\hline Substrate concentration & $S$ & $\mathrm{~g} / 1$ \\
\hline Glucose concentration & $G$ & $\mathrm{~g} / 1$ \\
\hline Acetate concentration & $A$ & $\mathrm{~g} / 1$ \\
\hline Product concentration / activity & $P$ & $\mathrm{~g} / 1 /$ normalised units \\
\hline Oxygen uptake rate & OUR & $\mathrm{mol} / \mathrm{min}$ \\
\hline Carbon dioxide emission rate & $C E R$ & $\mathrm{~mol} / \mathrm{min}$ \\
\hline Temperature & $T$ & ${ }^{\circ} \mathrm{C}$ \\
\hline Total pressure & $P$ & Atm \\
\hline Optical density at $650 \mathrm{~nm}$ & $O D_{650}$ & Absorption units \\
\hline \multicolumn{3}{|l|}{ Organism parameters } \\
\hline Specific glucose uptake rate & $q_{g}$ & $g /(g \cdot h)$ \\
\hline Specific oxygen uptake rate & $q_{o}$ & $g /(g \cdot h)$ \\
\hline Specific acetate production rate & $q_{a}$ & $g /(g \cdot h)$ \\
\hline Specific acetate uptake rate & $q_{a}^{c}$ & $g /(g \cdot h)$ \\
\hline \multicolumn{3}{|l|}{ Controller parameters } \\
\hline Cycle time & $t_{\text {cycle }}$ & $\mathrm{s}$ \\
\hline Pulse time & $t_{p}$ & $\min$ \\
\hline Control time & $t_{c}$ & $\min$ \\
\hline Observation time & $t_{o}$ & $\min$ \\
\hline Pulse feed rate modifier & $\gamma_{p}$ & - \\
\hline Pulse response feed rate modifier & $\gamma_{i}$ & - \\
\hline Probing controller gain & $\kappa$ & - \\
\hline Pulse amplitude & $F_{\text {pulse }}$ & as per $F$ \\
\hline Feed rate change after a pulse & $\Delta F$ & as per $F$ \\
\hline Dissolved oxygen reaction level & $D O_{\text {reac }}$ & $\%$ \\
\hline Dissolved oxygen pulse response & $D O_{\text {pulse }}$ & $\%$ \\
\hline Dissolved oxygen precision tolerance & $D O_{t o l}$ & - \\
\hline Dissolved oxygen stability tolerance & $\delta$ & $\%$ \\
\hline Number of previous cycles checked for stability & $n$ & - \\
\hline
\end{tabular}

Table 3.1 Parameter names, symbols and units 


\section{Background}

\subsection{Bioreactors and fed-batch processes}

The bioreactor, also referred to as the fermentor, can be considered the heart of a biotechnical process. It is where, through biochemical reactions, the desired product of the process is formed. Bacteria and fungi as well as insect and mammalian cells can all be used in bioprocesses, each having their own advantages and disadvantages.

Many different types of bioreactors exist today, such as unstirred vessels, stirred vessels, bubble columns, airlift reactors, membrane reactors, fluidised beds and packed beds (Lidén 2002, p. 274), however today almost all bioreactors are stirred tanks (Nielsen and Villadsen and Lidén 2003, p. 340).

Bioreactors can employ different modes of operation; batch, continuous and fedbatch. Batch mode indicates that no flows to or from the reactor exist; the reactor is filled with all necessary substrates and the process is then allowed to go on until the reactor is emptied and the desired product is recovered. Continuous mode uses the opposite approach and keeps the process at steady state. This means that flows both to and from the reactor exist during the process, where substrate is fed into the reactor at the same rate as it is consumed while the flow from the reactor contains the desired product of the process. Fed-batch mode is similar to batch mode but means that flows either to or from the reactor are utilised. In these definitions, gas flows to provide the reactor with oxygen and to lead away gaseous by-products such as carbon dioxide, as well as addition of acid or base to achieve the desired $\mathrm{pH}$, are ignored. A fed-batch reactor of the type used in this project is illustrated in figure 4.1.

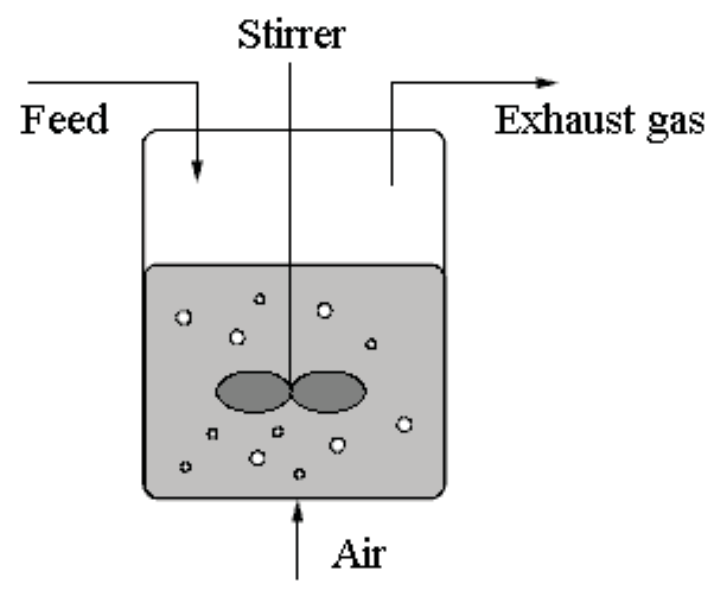

Figure 4.1 A fed-batch process in a stirred tank with an in-flow of feed (Åkesson 1999, p. 16 ), which is the typical mode of operation for a modern bioprocess and is used in this project.

Batch processes are flexible and safe but not very efficient, while continuous processes are more efficient but can have problems due to infection and mutation of the microorganism to a non-producing strain. Fed-batch processes are intended to utilise the advantages of batch processes while achieving a better efficiency, meaning that they combine the advantages of batch and continuous operation, as well as some of the disadvantages although the advantages normally outweigh these. Fed-batch pro- 
cesses also have the advantage from an automation point of view, that the process can be controlled through manipulation of the feed rates (Nielsen and Villadsen and Lidén 2003 , p. 341). A typical industrial bioprocess today is a fed-batch process (Lidén 2002, p. 273).

The fermentation medium in a bioreactor should be controlled in order to provide an optimal environment for the bacteria. The optimisation problem for a continuous process consists in finding an operating point (a set of values for the state variables) which is optimal for the process. For a fed-batch process the optimisation problem is more difficult, as the state of the process and thus the optimal operating point is time-dependent (Lidén 2002, p. 275).

\subsection{Substrate metabolism in cells}

When substrate is taken up by the microorganism, it can follow several different metabolic pathways. Depending on the state of the microorganism, different pathways will be more or less active. This is shown in figure 4.2, where the glucose metabolism of the bacterium $B$. subtilis under different conditions is displayed.

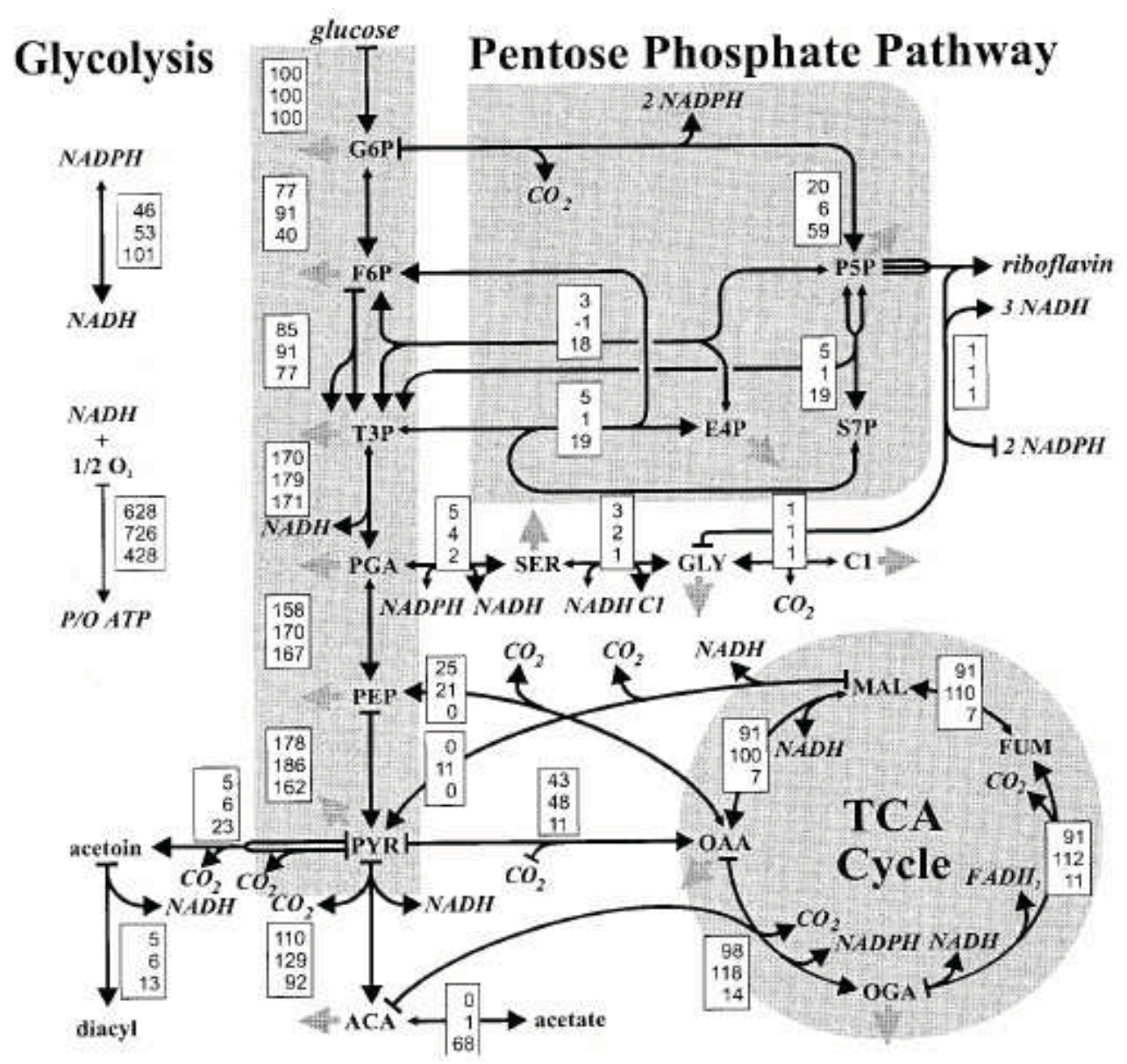

Figure 4.2 Metabolic flux distribution in a carbon-limited (top entry in boxes), nitrogenlimited (middle) and phosphate-limited (bottom) chemostat culture of B. subtilis. Fluxes are relative to the specific glucose consumption rate (set to 100). (Dauner and Storni and Sauer 2001, p. 7312) 
The oxidative metabolism, of which the TCA cycle is a part, has a maximum rate at which it can operate due to limitations in the respiratory capacity of the cell. As seen in figure 4.2, when carbon exists in excess the pathways leading to the production of acetate and acetoin are considerably more active than if the organism is glucose-limited. This is because of the rate limitation in the oxidative metabolism; the TCA cycle will at most take up pyruvate (PYR) at a certain rate, the excess production of which must be dealt with in other ways which do not require oxygen. Therefore it is converted into acetate and acetoin which are transported out from the cell into its surroundings. This is called fermentative metabolism and requires no oxygen (Nielsen and Villadsen and Lidén 2003, pp. 24-30). The rate limitation in the oxidative metabolism is illustrated in figure 4.3.

A

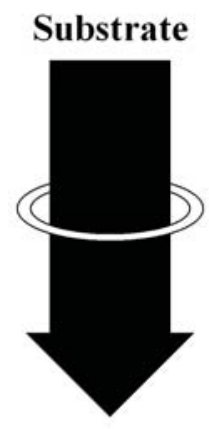

$\mathrm{CO}_{2}$
B

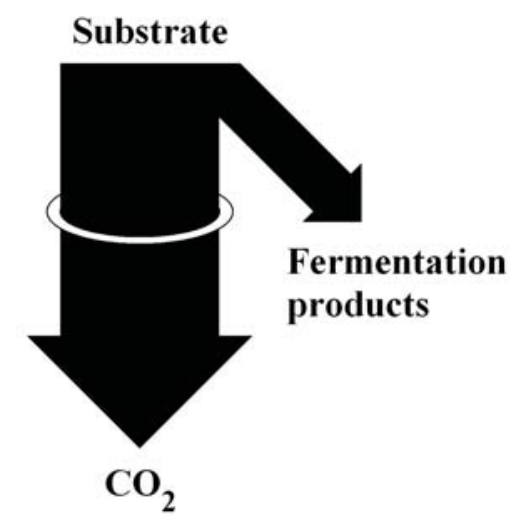

Figure 4.3 A simple illustration of the limitation in oxidative metabolism. In A, the respiratory capacity is not limiting and only the oxidative pathway is active. In B the respiratory capacity has been exceeded and some of the glucose will go into the fermentative pathway. (Nielsen and Villadsen and Lidén 2003, p. 257)

The stoichiometry for the oxidative and fermentative glucose metabolism are as shown in equations 4.1 and 4.2. In these equations, water and the nitrogen source are not included. (Nielsen and Villadsen and Lidén 2003, p. 258)

Oxidative glucose metabolism:

$$
\gamma_{1} X+\beta_{11} \mathrm{CO}_{2}-S-\alpha_{12} \mathrm{O}_{2}=0
$$

Fermentative glucose metabolism:

$$
\gamma_{2} X+\beta_{21} C O_{2}+\beta_{22} P-S=0
$$

The product $P$ in equation 4.2 is the fermentation product(s), which can differ from organism to organism. E. coli produces acetate and other bacteria (and human muscle cells) produce lactic acid while the yeast $S$. cerevisiae produces ethanol (Lidén 2002, p. 273). The overflow products of B. licheniformis are not known in detail but believed to be the same as those of the related species $B$. subtilis; acetate and acetoin (Voigt et al. 2004, p. 1469).

It should be noted that when the organism is oxygen-limited, no oxidative metabolism will occur and all of the substrate which is taken up will go through the fermentative metabolic pathway and be converted into fermentation products (fully fermentative metabolism). When fermentative metabolism occurs due to the presence 
of excess substrate rather than due to oxygen limitation in the system these reactions are labelled as overflow metabolism, as it occurs when there is an overflow of certain metabolites, and the products as overflow products.

In an industrial bioprocess, overflow metabolism is typically undesired as the overflow products tend to inhibit growth of the microorganism (except when an overflow product is the desired product of the process). Organic acids such as acetic and lactic acid exist in equilibrium between their dissociated and undissociated forms in the medium ( $\mathrm{HA} \rightleftharpoons \mathrm{H}^{+}+\mathrm{A}^{-}$) and their inhibition mechanism is due to the undissociated form being uncharged and thereby able to diffuse through the cell membrane to the interior of the organism, where it dissociates. This leads to a net influx of protons into the organism, which it must expend energy to counteract in order to avoid a decreased $\mathrm{pH}$ in its interior (Nielsen and Villadsen and Lidén 2003, pp. 18-19).

\subsection{Probing control of the feed rate in fed-batch fermentations}

To obtain maximal microbial growth, overflow metabolism and oxygen limitation should be avoided without using a lower feed than necessary. This means that the feed rate, $F$, should be at such a level that the specific substrate uptake rate, $q_{s}$, is kept at or just below the critical level for overflow metabolism , $q_{s, c r i t}$, while $D O$ is kept above zero. The challenges in achieving feed just below $q_{s, c r i t}$ in a fed-batch process are twofold:

- $q_{s, c r i t}$ is often poorly known and may change during a fermentation due to metabolic shifts (Åkesson 1999, paper I, pp. 596-597).

- As the biomass concentration and the total volume increase during the process, the feed rate must be increased to give the same feed rate per amount of biomass even if $q_{s, c r i t}$ remains constant.

It is possible, based on empirical studies of the process, to design a static feed curve to control the fermentation during the outgrowth phase, which can give acceptable results. However, this approach uses no feedback and therefore does not take variations in the process into account. Because of the varying $q_{s, c r i t}$, it is desirable to use a control strategy which directly tests whether or not $q_{s, c r i t}$ has been exceeded no matter what level it is at and which can adjust $F$ to suit the current conditions.

Probing control of feed rate is a control strategy related to the area of dual control, as it injects probing signals (pulses) into the process and analyses the effects of these although the purpose of these pulses is not to achieve better control characteristics in future steps as in dual control (Wittenmark 1995, p. 1) but merely to make conclusions regarding the current state of the system and thus find the optimal current control signal. By exploiting the characteristic saturation in the specific oxygen uptake rate $q_{o}$ when $q_{s, c r i t}$ is reached, the probing controller studies the $D O$ response to pulses in $F$ to determine the position of the current $q_{s}$ relative to $q_{s, c r i t}$. This means that it can be seen as a type of extremum control with the optimal operating point being just below $q_{s, c r i t}$, although it detects a saturation rather than a maximum. This strategy has been employed successfully in fermentations utilising among others $E$. coli (Åkesson 1999; Velut 2005; de Maré 2006; Henes and Sonnleitner 2007), V. cholerae (de Maré 2006) and S. cerevisiae (Henes and Sonnleitner 2007).

Probing control has the desirable trait that the only sensor equipment it requires is a $D O$ probe, which is standard equipment for bioreactors. This means that no addi- 
tional sensors need to be added to implement probing control in an existing bioprocess (de Maré 2006, p. 20).

\subsection{Bacillus licheniformis}

Bacillus licheniformis is a Gram-positive organism which can be found in soils and plant material and is able to form endospores. It has not been found to be pathogenic for either animals or plants. B. licheniformis belongs to the B. subtilis group (group II) of the genus Bacillus, in which several other well-known species can be found, such as Bacillus anthracis, Bacillus cereus and of course Bacillus subtilis (Veith et al. 2004, pp. 204-205). Unlike most other bacilli, which are predominantly aerobic, B. licheniformis is facultatively anaerobic (Rey et al. 2004, p. 2).

$B$. licheniformis is extensively used for large-scale industrial production of enzymes, as it can produce and secrete large quantities of proteins (Schallmey and Singh and Ward, p. 7). It has been used in this role for several decades, in the production of for instance proteases, $\alpha$-amylase, penicillinase, cycloglucosyltransferase, pentosanase, $\beta$-mannanase and several pectinolytic enzymes. Certain strains of $B$. licheniformis have been used to produce peptide antibiotics as well as various specialty chemicals such as citric acid and inosine (Rey et al. 2004, p. 2).

In addition to its industrial uses, $B$. licheniformis has shown potential as a biocontrol agent, as some isolates have an antifungal effect against various pathogenic fungi affecting vegetable crops, cereal grasses and coffee among others (Neyra et al. 1996, pp. 453-454).

\subsection{Novozymes}

Novozymes is an international biotech-based company, created in 2000 in a split of Novo Nordisk. It is the world's largest producer of industrial enzymes, with $47 \%$ of the market in 2009. The company's headquarters is located in Bagsværd, Denmark, although it employs about 5000 people in 30 different countries.

Although Novozymes has three main business areas, industrial enzymes, microorganisms and biopharmaceutical ingredients, the industrial enzymes are by far the most important products and make up $92 \%$ of the company's sales. The enzymes produced can be divided into the following categories (Wikipedia 2010).

- Detergent enzymes, used in laundry detergents and dish washing machines.

- Technical enzymes, mainly for the starch, textile and bioethanol industries.

- Food enzymes, for a wide variety of food industries such as the baking, brewing and dairy industries.

- Feed enzymes, for the animal feed industry. 


\section{Theory}

\subsection{A model of microbial growth and production in fed-batch fermentations}

The growth of microorganisms in a fermentation and their production of the desired product is dependent on what substances are present in the fermentor and in what concentrations. A simple model is used here to describe such a process, not taking into account changes in gene expression which may occur in the microorganisms during a fermentation. Only the concentrations of substrate, oxygen, biomass, product and inhibiting substances will be considered here.

Fed-batch bioprocesses operate at non-steady state, meaning that the concentrations of various substances vary over time due to changes in liquid volume $(V)$, feed rate $(F)$, gas flow rates $\left(Q_{i}\right)$, specific uptake/emission rates $\left(q_{i}\right)$ and the specific biomass growth rate $(\mu)$. In a fed-batch fermentation with inlet flows of substrate and air and an outlet flow of gas, the different states in the fermentor can be described as in equations 5.1-5.5 (Åkesson 1999, paper V, p. 5; Nielsen and Villadsen and Lidén 2003, pp. 425-427).

$$
\begin{array}{r}
\frac{d V_{\text {medium }}}{d t}=F \\
\frac{d(V X)}{d t}=V X \cdot \mu(S, A) \\
\frac{d(V S)}{d t}=F S_{\text {in }}+q_{s}(S) \cdot V X \\
\frac{d(V O)}{d t}=K_{L} a\left(N, Q_{\text {gas }, \text { in }}\right) \cdot V\left(O^{*}-O\right)+q_{o}(S, A) \cdot V X \\
\frac{d(V P)}{d t}=q_{p}(S, A) \cdot V X
\end{array}
$$

$K_{L} a$ is the volumetric mass transfer coefficient, in this case for oxygen. $O^{*}$ is the dissolved oxygen concentration at equilibrium with the oxygen in the gas phase, which is here assumed to be constant for simplicity although it does in fact vary with the oxygen concentration in the gas phase (de Maré 2006, paper II, p. 6).

\section{Specific rate of microbial growth}

The specific growth rate, $\mu$, is dependent on several factors. For cell growth on a single limiting substrate however, at low values of $S$ the specific growth rate is proportional to $S$, but as it increases the specific growth rate reaches an upper limit. The Monod model, seen in equation 5.6, is the simplest and most frequently used mathematical model for the specific growth rate (Nielsen and Villadsen and Lidén 2003, p. 246).

$$
\mu=\mu_{\max } \frac{S}{S+K_{S}}
$$


$K_{S}$ is a measure of the organism's affinity for the substrate and, as can be seen in equation 5.6, is equal to the concentration of the limiting substrate at which the specific growth rate is half of its maximum value.

When glucose is the limiting substrate, $K_{s}$ is normally in the milligram per liter range (Nielsen and Villadsen and Lidén 2003, p. 246). As displayed in figure 5.1, this means that $\mu$ reaches $\mu_{\max }$ at very low concentrations and that there is only a small concentration range where $\mu$ is neither close to zero nor close to $\mu_{\max }$.

It is possible to use another substrate than the preferred one, for instance organisms such as $E$. coli can consume the acetate they have produced in overflow metabolism. The uptake mechanism for such an alternate substrate can also be described using the Monod model, but the uptake will only occur when the preferred substrate becomes limiting (as shown in figure 5.3) (Åkesson 1999, paper V, p. 4).

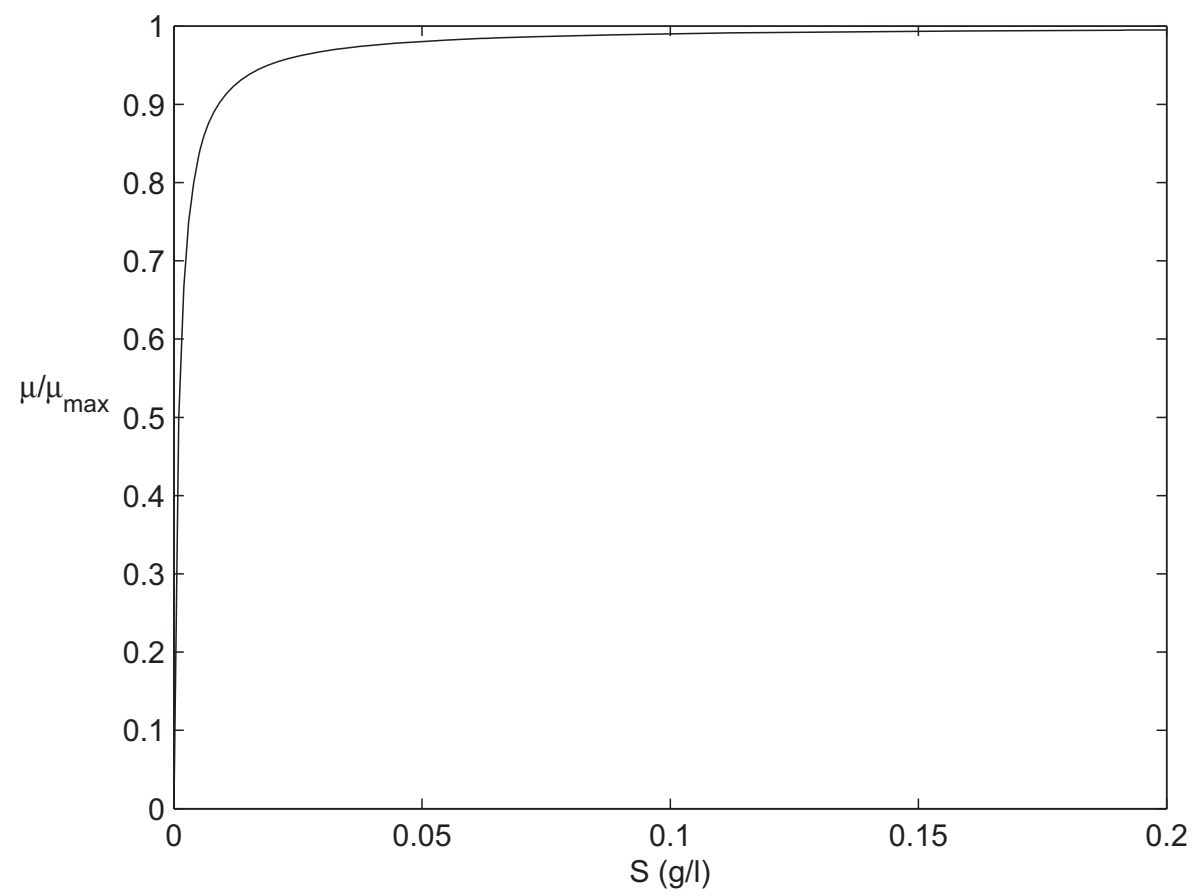

Figure 5.1 Illustration of the Monod model for biomass growth with glucose as the limiting substrate $\left(K_{S}=0.001 \mathrm{~g} / 1\right)$.

Microbial growth can be inhibited both by high concentrations of substrate and by the presence of a metabolic product, such as acetate. These effects can be described by adding additional terms to the Monod model, as follows in equation 5.7 (substrate inhibition) and 5.8 to 5.9 (product inhibition).

$$
\begin{array}{r}
\mu=\mu_{\max } \frac{S}{S^{2} / K_{i}+S+K_{S}} \\
\mu=\mu_{\max } \frac{S}{S+K_{S}} \frac{1}{1+P / K_{i}} \\
\mu=\mu_{\max } \frac{S}{S+K_{S}}\left(1-\frac{P}{P_{\max }}\right)
\end{array}
$$

Although the inhibition effects of acetate on the growth of B. licheniformis are 
not known, for $E$. coli $\mu$ decreases logarithmically with increasing acetate concentration (Luli and Strohl 1990, p. 1006). Product inhibition using the models shown in equations 5.8 and 5.9 influences $\mu$ as illustrated in figure 5.2.

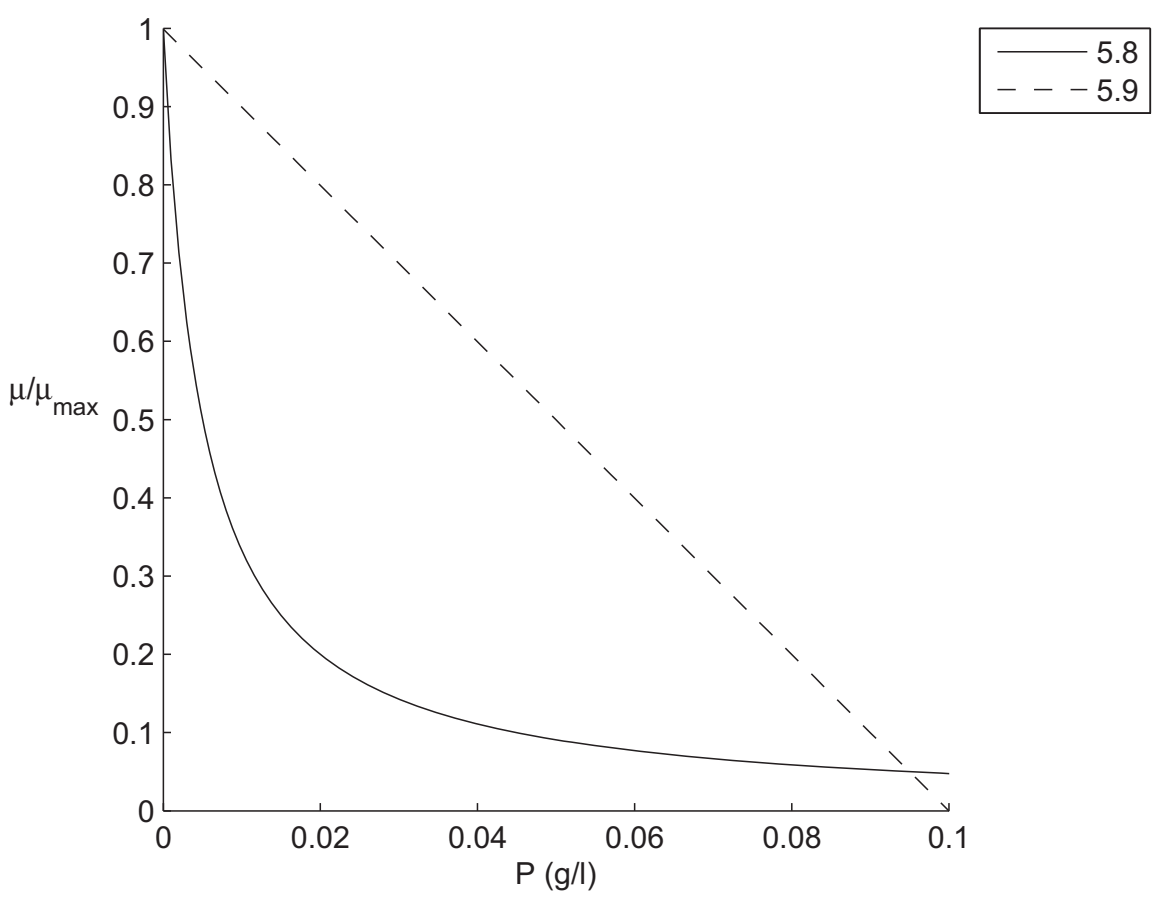

Figure 5.2 Illustration of inhibition effects according to expanded Monod models ( $S=$ $\left.1 \mathrm{~g} / 1, K_{S}=0.001 \mathrm{~g} / 1, K_{i}=0.005 \mathrm{~g} / 1, p_{\max }=0.1 \mathrm{~g} / 1\right)$.

\section{Overflow metabolism}

Normally the specific oxygen uptake rate of cells, $q_{o}$, increases with the specific substrate uptake rate $q_{g}$, but as described in section 4.2 the specific oxygen uptake rate $q_{o}$ is limited by the respiratory capacity of the biomass. The maximum specific oxygen uptake rate $q_{o, \max }$ is described in equation 5.10, but as $O$ is typically much larger than $K_{o}$ the maximum specific oxygen uptake rate becomes equal to $k_{o}$. (Nielsen and Villadsen and Lidén 2003, p. 258)

$$
-q_{o, \max }=k_{o} \frac{O}{O+K_{o}}
$$

The limitation in specific oxygen uptake rate does not limit the specific substrate uptake rate although oxidative metabolism of the substrate requires oxygen, rather the specific substrate uptake rate can be specified as in equation 5.11. (Nielsen and Villadsen and Lidén 2003, p. 258)

$$
-q_{s}=k_{s} \frac{S}{S+K_{s}}
$$

The $q_{s}$ value corresponding to $q_{o, \max }$ is referred to as the critical specific substrate uptake rate, $q_{s, c r i t}$ and is defined as in equation 5.12, where $\alpha_{12}$ is the amount of oxygen consumed per amount of glucose in oxidative metabolism as shown in equation 
4.1. When $q_{g}$ goes above $q_{g, \text { crit }}$ acetate is produced and when $q_{g}$ is below it acetate can be consumed, the latter of which requires oxygen (Akesson 1999, paper V, p. 4). The rate of oxidative metabolism $v_{o x}$ is expressed in equation 5.13 (Nielsen and Villadsen and Lidén 2003, p. 259) and the rate of fermentative metabolism $v_{f e r}$ due to overflow is expressed in equation 5.14 .

$$
\begin{array}{r}
q_{s, \text { crit }}=\frac{q_{o, \max }}{\alpha_{12}} \\
v_{o x}=\min \left(q_{s}, q_{s, c r i t}\right) \\
v_{f e r}=\max \left(q_{s}, q_{s, c r i t}\right)-q_{s, c r i t}
\end{array}
$$

In the context of this project, the preferred substrate for the organism is glucose, meaning that $q_{s}$ is from here on referred to as $q_{g}$. The feed rate which corresponds to $q_{g, c r i t}$ is termed $F_{\text {crit }}$.

Figure 5.3 gives a graphic summary of overflow metabolism as described in this section, by displaying relations between uptake and production rates of the relevant substances.

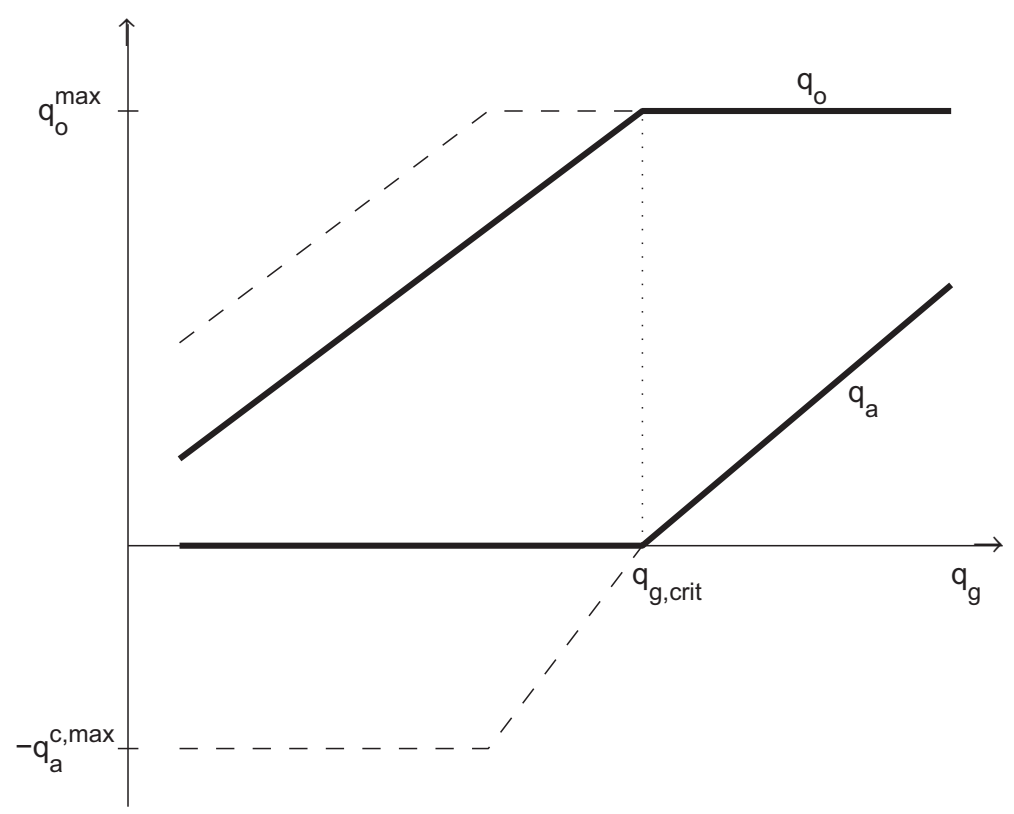

Figure 5.3 Relations between specific rates of glucose uptake, $q_{g}$, oxygen uptake, $q_{o}$, acetate production, $q_{a}$ and acetate uptake, $q_{a}^{c}$, in a glucose-fed E. coli fermentation. Dashed lines show potential acetate uptake and its effect on $q_{o}$ when acetate is present in the medium ( $\AA$ kesson 1999, p. 20). 


\subsection{Probing control}

\section{Detection of overflow metabolism}

The principle behind probing control in fed-batch processes with a glucose feed is to detect the saturation in the respiration which occurs when $q_{g}$ reaches $q_{g, c r i t}$ (see figure 5.3). This can be done by superimposing pulses over the baseline of the feed rate $F$, which will lead to temporary changes in $q_{g}$. If $q_{g}$ is below $q_{g, \text { crit }}$, such a pulse will give a response in the dissolved oxygen saturation $(D O)$ signal, as the fraction of the respiratory capacity required to metabolise glucose and thereby the oxygen uptake rate will change, as illustrated in figure 5.3. If $q_{g}$ is at or above $q_{g, c r i t}$, the oxidative capacity is saturated so no change in oxygen uptake will occur, meaning that no $D O$ response will be seen. Such feed pulses must be long enough to be seen through the glucose and oxygen dynamics of the system, but it is also desirable to keep them short to make the control loop faster and because the process should be disturbed as little as possible by these pulses ( $\AA$ kesson 1999, p. 24). Pulses in the probing control strategy are illustrated in figure 5.4. It would also be possible to return $F$ to the value before the pulse for a certain time, before determining the new feed rate. This variation is illustrated in figure 5.5.

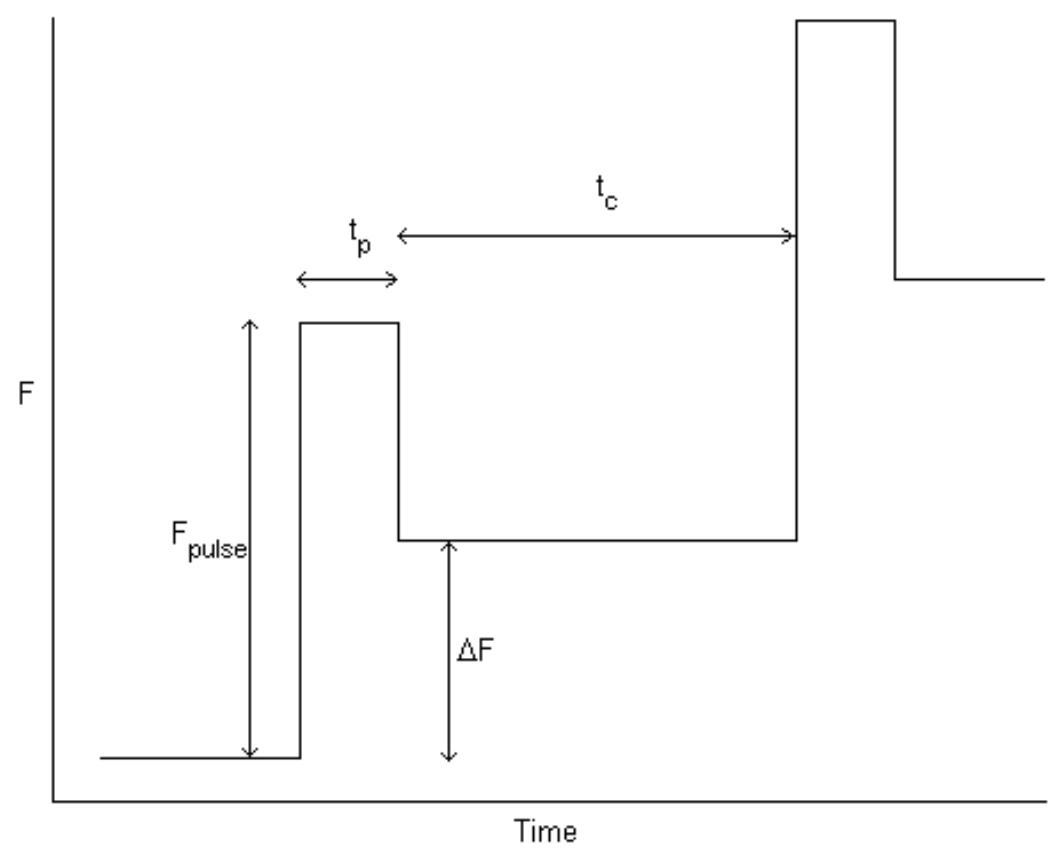

Figure 5.4 Illustration of a probing pulse, showing the parameters involved. These are up pulses; a probing control strategy can use up and/or down pulses.

\section{$D O$ control and measurement}

Dissolved oxygen saturation, $D O$, is in practice given by a probe measuring the dissolved oxygen tension, which is proportional to the oxygen partial pressure. A dissolved oxygen saturation of $100 \%$ corresponds to the maximum value of the dissolved oxygen tension, which is reached when the oxygen partial pressure is in equi- 


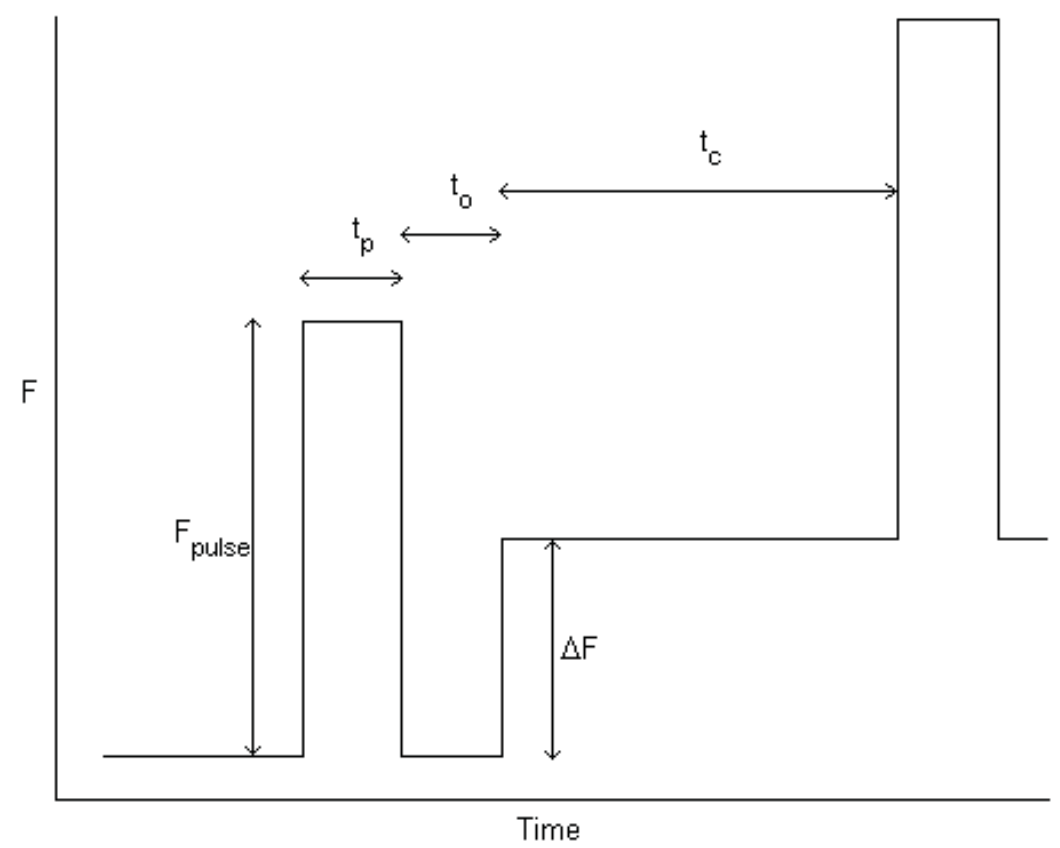

Figure 5.5 Illustration of a probing pulse with a delayed change of the feed rate baseline, showing the parameters involved.

librium with the oxygen pressure in the gas phase $\left(O=O^{*}\right)$.

Throughout a fermentation it is desirable to avoid oxygen limitation $(D O=0)$, as this can have a negative impact on the microbial growth and production. This can be achieved using a simple PI controller on the form shown in equation 5.15, controlling the agitator speed, aeration rate, gas pressure and/or the oxygen content of the inlet gas to keep $D O$ at a predetermined setpoint for the entire process despite the nonstationary nature of a fed-batch process.

$$
u(t)=P(t)+I(t)=K_{c}\left(D O_{s p}-D O_{p v}\right)+\frac{K_{c}}{T_{i}} \int^{t}\left(D O_{s p}-D O_{p v}\right) d t
$$

The volumetric mass transfer coefficient for oxygen, $K_{L} a$ (see equation 5.4), influences the process dynamics and it can therefore be of interest to use it as a scheduling variable for controller parameters. It is possible to assume a linear relation between the stirrer speed $N$ and $K_{L} a$ within a set range, meaning that $N$ itself can be used as the scheduling variable for $D O$ control utilising $N$ as the control parameter (Åkesson 1999, paper III, p. 3).

In a discrete controller, it is easy to utilise an iterative process to calculate the integral part, giving a controller on the form shown in equation 5.16 .

$$
u(i)=P(i)+I(i)=K_{c}\left(D O_{s p, i}-D O_{p v, i}\right)+I(i-1)+\frac{K_{c}}{T_{i}}\left(D O_{s p, i}-D O_{p v, i}\right)
$$




\section{Different probing strategies and controller responses}

There are several variations on the probing feeding strategy, although they all share the same central principles. Some examples are given in table 5.1.

\begin{tabular}{lll}
\hline Variants & Comments & Examples \\
\hline $\begin{array}{l}\text { Varying between up } \\
\text { and down pulses }\end{array}$ & $\begin{array}{l}\text { Pulse direction determined by } \\
\text { whether the last pulse gave a re- } \\
\text { sponse or not }\end{array}$ & Akesson 1999 \\
Only using up pulses & $\begin{array}{l}\text { Simplified method which can im- } \\
\text { prove performance }\end{array}$ & Akesson and Hagander 2000 \\
Only using down & $\begin{array}{l}\text { Suitable when overfeeding is par- } \\
\text { ticularly undesirable }\end{array}$ & Henes and Sonnleitner 2007 \\
pulses & $\begin{array}{l}\text { Down pulses used after maxi- } \\
\text { mum oxygen transfer capacity is } \\
\text { First using up pulses }\end{array}$ & Velut 2005 \\
then down pulses & $\begin{array}{l}\text { reached, to achieve a slight ex- } \\
\text { growth temperature-regulated }\end{array}$ & \\
&
\end{tabular}

Table 5.1 Variants of probing control.

For a strategy employing both up and down pulses, such as the original probing control strategy for feed rate, up pulses can be used for testing whether the feed should be increased while down pulses are used to test if the feed should be decreased. A strategy employing only pulses in one direction must use the response to that pulse type to determine whether the feed should increase or decrease.

\section{Details on the chosen strategy}

Although the probing control feeding strategy has developed significantly since it was first formulated, for instance by combining probing control with the temperatureregulated fed-batch technique (Velut 2005), the basis for the probing strategy used in this project is that described in (Åkesson 1999, Paper V). This is because it is simple and expected to be suitable for implementation in a new process as it performs both up and down pulses which should give more information about the current state of the process than if using only one type of pulse. Another reason for not using temperature-limitation techniques is that the existing process utilises a predefined temperature ramp. The details of the chosen strategy are described in this section.

As mentioned in table 5.1, this strategy employs both up and down pulses. The basic principle for the controller's decision to change $F$ is shown in table 5.2.

\begin{tabular}{llll}
\hline Up pulse & Down pulse & State of system & Controller decision \\
\hline Response & Response & $F$ far below $F_{\text {crit }}$ & Increase $F$ \\
No response & Response & $F$ at or just below $F_{\text {crit }}$ & Keep $F$ constant \\
No response & No response & $F$ above $F_{\text {crit }}$ & Decrease $F$ \\
\hline
\end{tabular}

Table 5.2 The principle behind the probing controller's decision-making.

The algorithm determining whether the feed rate should be increased, remain the same or be decreased and in which direction (up or down) the next pulse should be is illustrated in figure 5.6. As seen there, up pulses can increase $F$ while down pulses can decrease it. If the last pulse has given a response this indicates that $F$ has been below $F_{c r i t}$, so the next pulse becomes an up pulse. Similarly, if the last pulse has given no response this indicates that $F$ has been above $F_{c r i t}$ so the next pulse becomes a down pulse. 


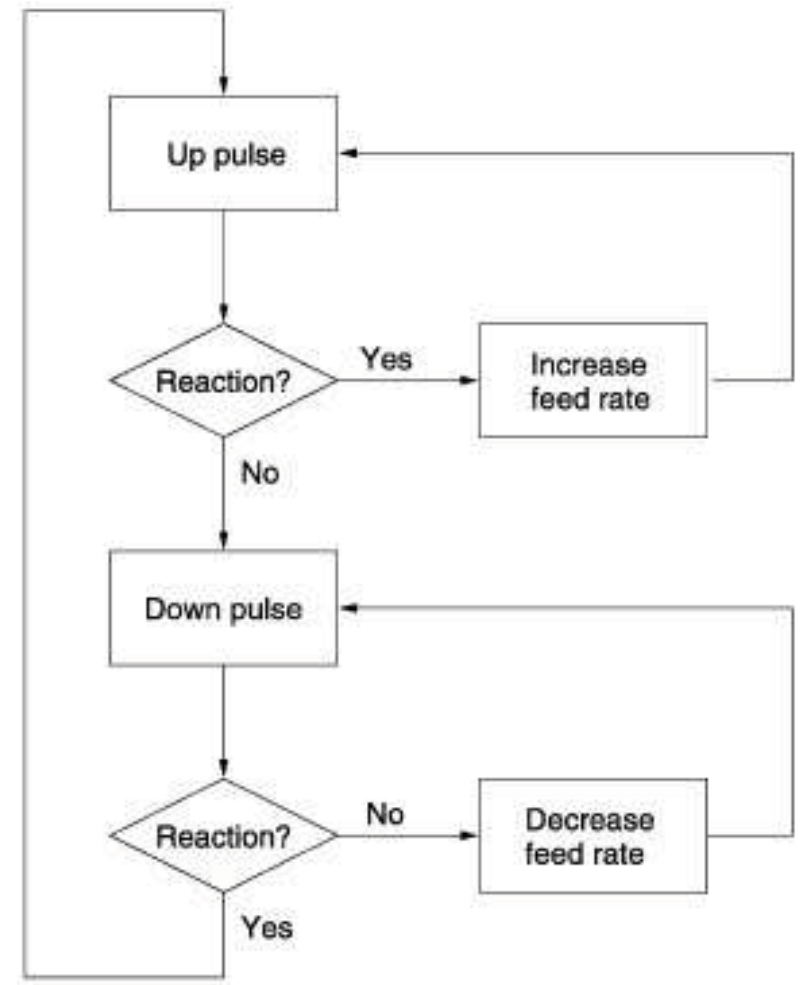

Figure 5.6 Flow diagram for the control algorithm. (Åkesson 1999, paper V, p. 9)

To determine if a pulse has given cause to a $D O$ response, the highest amplitude of the change in $D O$ during a pulse, $D O_{\text {pulse }}$ is compared to a defined reaction level, $D O_{\text {reac }}$. If $D O_{\text {pulse }}>D O_{\text {reac }}$, the pulse is said to have given a response. This is illustrated in figure 5.7 .

The amplitude of a pulse must be sufficient that the oxygen response can exceed $D O_{\text {reac }}$, but it is undesirable to make pulses which have a too large influence on the system, for instance through making $D O$ reach zero during up pulses. (Akesson 1999 , paper V) uses a pulse amplitude proportional to the current feed rate using the factor $\gamma_{p}$ as shown in equation 5.17 and recommends a value of $\gamma_{p}$ as shown in equation 5.18 .

$$
\begin{array}{r}
F_{\text {pulse }}=\gamma_{p} \cdot F \\
\gamma_{p} \approx 4 \cdot \frac{D O_{\text {reac }}}{D O^{*}-D O_{s p}}
\end{array}
$$

The $D O$ response to a pulse will be lower when $F_{c r i t}$ is passed during the pulse than if $F$ is below $F_{c r i t}$ both before and during the pulse, as in the former situation only a part of the change in $q_{g}$ will lead to a change in $q_{o}$ (see figure 5.3). It is therefore suitable to make the change in $F$ after a pulse, $\Delta F$, depend on the amplitude of the $D O$ response to the pulse. (Åkesson 1999, paper V, p. 18) shows that with the process model used there, a sufficient condition for convergence to a value of $F$ which gives $q_{g}$ close to but below $q_{g, c r i t}$ is given by equations 5.19 and 5.20 for up and down pulses respectively when the gain $\kappa \leq 1$. 

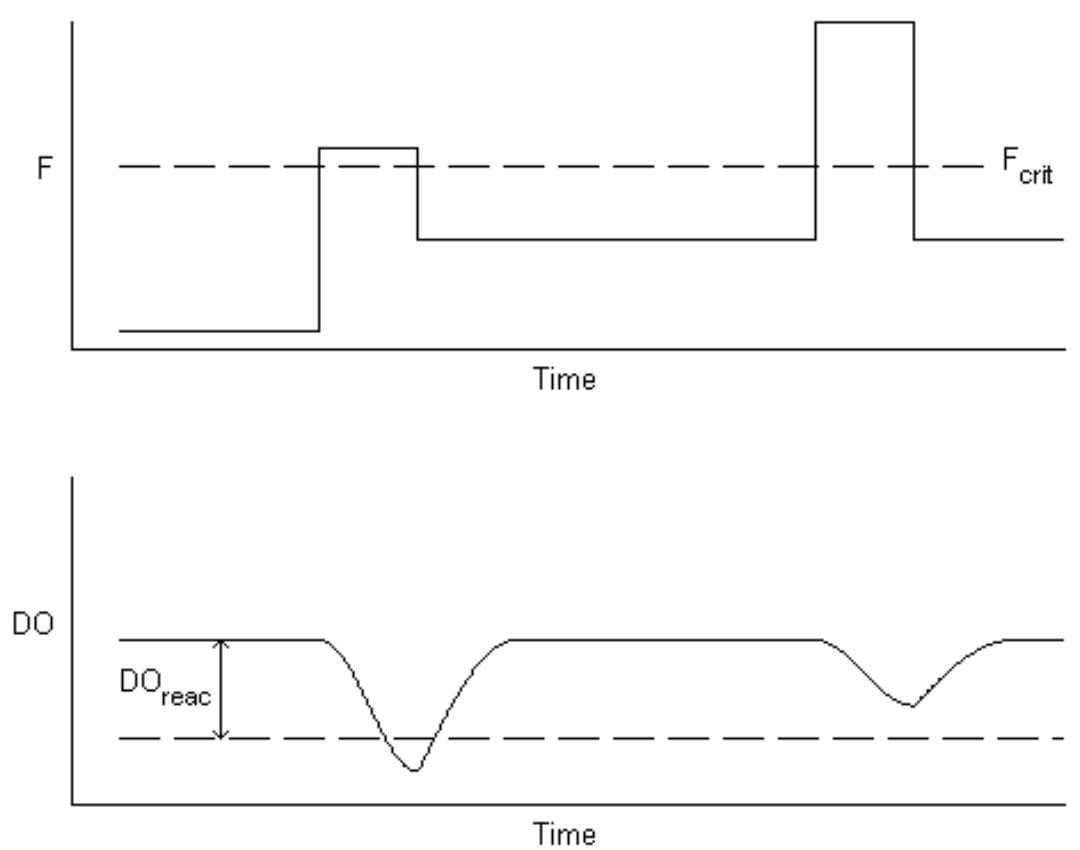

Figure 5.7 Examples of $D O$ behaviour during pulses. The left pulse gives cause to a response, while the right one does not.

$$
\begin{array}{r}
\Delta F=\kappa \cdot \frac{\left|D O_{\text {pulse }}\right|}{D O^{*}-D O_{s p}} \cdot F \\
\Delta F=\left(\kappa \cdot \frac{\left|D O_{\text {pulse }}\right|}{D O^{*}-D O_{s p}}-\gamma_{p}\right) \cdot F
\end{array}
$$

These gains are limited by $0 \leq \Delta F \leq F_{\text {pulse }}$ for up pulses and $-F_{\text {pulse }} \leq \Delta F \leq 0$ for down pulses. Using a higher value of $\kappa$ means that the controller can react faster to changes and disturbances in the process. It also means that the highest specific growth rate which can be followed by the feed rate controller is increased.

As the behaviour of the $D O$ signal during a pulse is what determines the probing controller's reaction, it is desirable to make as accurate measurements of $D O$ as possible and to not have other effects influence the $D O$ signal. Therefore $D O$ control is switched off during pulses, as it would otherwise counteract any $D O$ response to the pulse. To avoid erroneous interpretations of $D O$ behaviour during a pulse, stability conditions for $D O$ can be set up which must be fulfilled before a new pulse can be performed. In (Åkesson 1999, paper V) the condition $\left|D O_{p v}-D O_{s p}\right|<D O_{t o l}$ is used.

Shorter intervals between pulses means that $F$ can be adjusted to the changing conditions of the process more often and is therefore desirable. The limit for this is how fast the PI regulator can return $D O$ to a value which fulfils the stability conditions after a pulse has been performed. Fast, well-tuned $D O$ control is therefore desirable to achieve more efficient probing control, but is not critical as long as stability at $D O_{s p}$ is eventually reached. 


\section{The process and the experimental setup}

At Novozymes, Bacillus licheniformis is used to produce recombinant proteins in fedbatch processes. The currently existing process control system regulates the feed rate to keep the dissolved oxygen level well above the point where it becomes limiting. Although this system avoids dissolved oxygen limitation in the system it does not ensure that overflow metabolism is avoided. Occasionally these processes crash and it is suspected that this is due to overflow metabolism. B. licheniformis is closely related to B. subtilis and is therefore believed to have similar overflow mechanisms.

\subsection{General information on the process}

The pilot scale fermentation process follows a typical fed-batch mode of operation. The bacteria are grown in repeatedly larger scales until the fermentation starts in the main tank. A sample containing bacteria from the strain which will be used is first thawed and the bacteria are moved to a plate with agar medium where they are allowed to grow. After the bacteria have grown into colonies, these are studied to ensure that there is no contamination by other types of bacteria and samples from them are transferred into a shake flask. There, the bacteria are grown for a couple of hours after which an inoculation tank is inoculated from the shake flask. The inoculation tank is similar to the main tank but uses other process parameters and a different, complex medium while the main tank uses a defined medium which contains all the necessary nutritients for the bacteria excluding glucose and nitrogen (added through $\mathrm{pH}$ regulation with base). After outgrowth and depletion of initial substrate, the main tank is inoculated from the inoculation tank and the main process is started. This startup procedure is illustrated in figure 6.1.

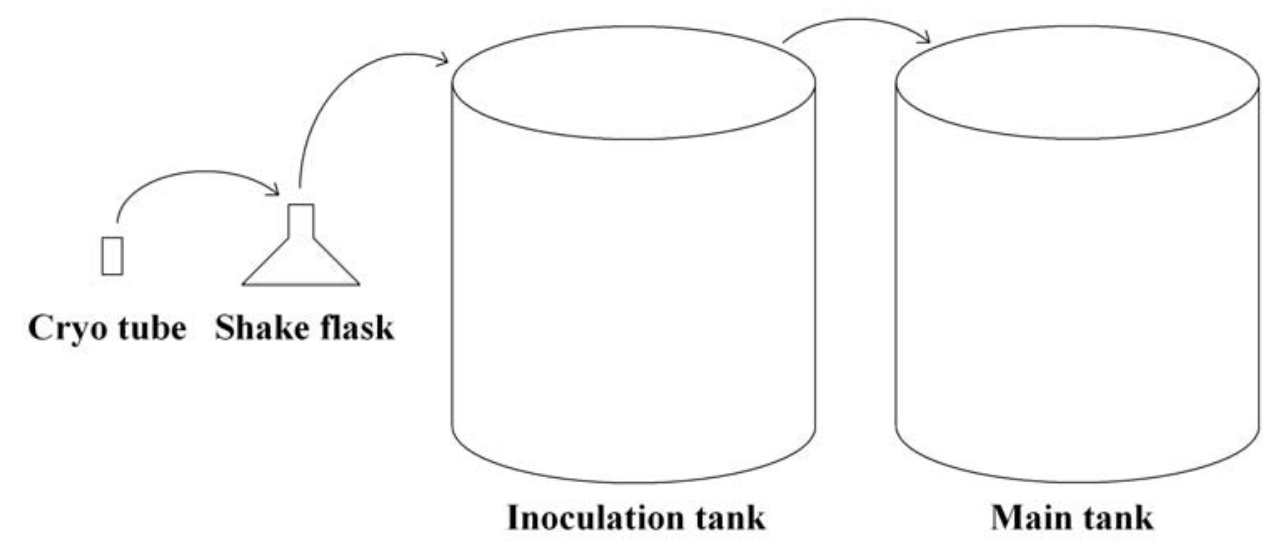

Figure 6.1 Illustration of the growth of the bacterial culture in preparation for the process in the main tank.

All nutritients required for the growth of the bacteria are present in the initial medium, except for glucose and the nitrogen source. Enough of the nitrogen source is added through $\mathrm{pH}$ regulation during the process that it can be assumed to exist in 
excess during the entire process. Glucose is added as a feed throughout the process, meaning that the growth and production of the microorganism will only be restricted by the access to glucose and other substrate limitation effects will not influence it.

In this project, 201 fermentations were run under the conditions specified in table 6.1 and $2.5 \mathrm{~m}^{3}$ fermentations under the conditions specified in table 6.2 (except where noted elsewhere). For a display of which batches were run in which fermentor during which experiment, see appendix A.

\begin{tabular}{ll}
\hline Parameter & Value \\
\hline Number of fermentors & 3 \\
Fermentor names (tags) & $107 \mathrm{M}, 108 \mathrm{M}, 109 \mathrm{M}$ \\
Process type & Fed-batch \\
Total fermentor volume & 201 \\
Initial volume & 81 \\
Agitator speed range & $400-1000 \mathrm{rpm}$ \\
Aeration rate range & $5-151 / \mathrm{min}$ \\
Feed composition & Glucose $(0.878 \mathrm{~kg} / 1,0.667 \mathrm{~kg}$ glucose $/ \mathrm{kg})$ \\
\hline
\end{tabular}

Table 6.1 Data for 201 fermentors used in small-scale experiments.

\begin{tabular}{ll}
\hline Parameter & Value \\
\hline Number of fermentors & 1 \\
Fermentor name (tag) & $32 \mathrm{C}$ \\
Process type & Fed-batch \\
Total fermentor volume & $2.5 \mathrm{~m}^{3}$ \\
Initial volume & $0.7 \mathrm{~m}^{3}$ \\
Agitator speed range & $100-275 \mathrm{rpm}$ \\
Aeration rate range & $500-15001 / \mathrm{min}$ \\
Feed composition & Glucose $(0.878 \mathrm{~kg} / 1,0.667 \mathrm{~kg}$ glucose $/ \mathrm{kg})$ \\
\hline
\end{tabular}

Table 6.2 Data for $2.5 \mathrm{~m}^{3}$ fermentor used in large-scale experiments.

The $D O$ probe used in the process is calibrated to show $100 \%$ just before the process is started, when the medium is oxygen saturated and the gas pressure in the fermentor is $2.3 \mathrm{~atm}$. During the process the gas pressure is $2 \mathrm{~atm}$, meaning that the $D O$ corresponding to an oxygen saturated medium, labelled $D O^{*}$, is $2 / 2.3 \cdot 100 \%$ $\approx 87 \%$.

In the 201 fermentations, $F_{s p}$ is calculated based on a weight measurement of the substrate tank. In the $2.5 \mathrm{~m}^{3}$ fermentations, $F_{s p}$ is determined using a flow meter. The feed is discontinuous in both scales, as it is added in discrete volumes. In the 201 fermentations $F$ is changed by varying the fraction of the time during which the feed pump is switched on (at its maximum), while in the $2.5 \mathrm{~m}^{3}$ fermentations the feed is entered in "shots" and both the volume of these and the time between them can be varied.

In all fermentations, the gas flow into the system consists of air which is passed through a sterile filter. This means that the oxygen partial pressure in this gas flow is constant at $21 \%$ of the total pressure. 


\subsection{Practicalities of controller implementation}

To employ a probing controller at the fermentation pilot plant, the control script is written in MATLAB on a computer connected to the process control system, a commercial system named DeltaV which controls all fermentations at the pilot plant. The controller can read the current setpoints and process values from DeltaV and write setpoints to DeltaV. This means that the programmed probing controller does not directly control process parameters; instead the control loops in DeltaV control the process using the setpoints given by the probing controller. This is illustrated in figure 6.2 .

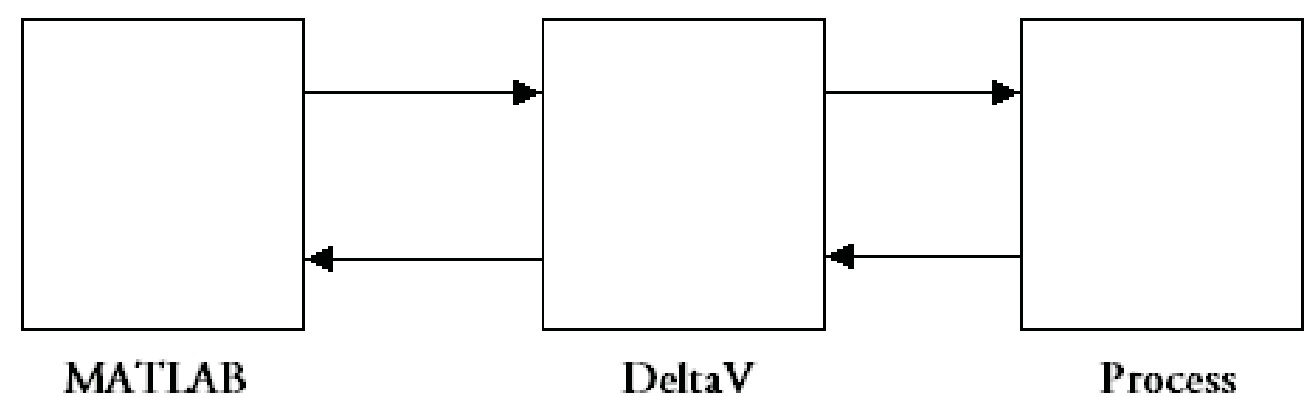

Figure 6.2 Overview of data flow in the process control system.

The controller is discrete with a set cycle time $t_{c y c l e}$. It operates through running a MATLAB script repeatedly; each iteration of this script being run is a cycle in the controller. The controller is started by running a separate MATLAB script which loads predefined parameters and initiates the iteration of the controller script.

The controller regulates three process parameters, $F, N$ and $Q_{g a s, i n} . T, P$ and $\mathrm{pH}$ are controlled by DeltaV, utilising ramps similar to those used in the existing control strategy (see figure 7.1). Although the MATLAB script reads several process parameters for data analysis purposes, the only process parameter utilised by the closed-loop probing controller is $D O$.

\subsection{Data sampling during experiments}

Several process parameters can be measured on-line during the fermentations. For the probing controller's purposes $D O$ is the only parameter which needs to be measured on-line, but many others are of interest to ensure that the fermentation is run under the proper conditions. Other parameters are relevant to evaluate the outcome of the experiment but cannot be measured on-line, so samples are taken from the fermentor. Table 6.3 shows all measured parameters, both on-line and off-line. For a detailed description of sampling procedures during the experiments, see appendix B. 


\begin{tabular}{ll}
\hline \multicolumn{2}{c}{ Parameters } \\
\hline Measured on-line & Measured off-line \\
\hline Dissolved oxygen saturation $(D O)$ & Optical density at $650 \mathrm{~nm}\left(O D_{650}\right)$ \\
Aeration rate $\left(Q_{\text {gas }, i n}\right)$ & Dry mass concentration $(X)$ \\
Agitator speed $(N)$ & Glucose concentration $(G)$ \\
Feed rate $(F)$ & Acetate concentration $(A)$ \\
Temperature $(T)$ & Product activity $(P)$ \\
Total pressure $(P)$ & \\
Substrate tank weight & \\
$\mathrm{O}_{2}$ uptake rate $(O U R)$ & \\
$\mathrm{CO}_{2}$ emission rate $(C E R)$ & \\
\hline
\end{tabular}

Table 6.3 Parameters measured during experiments. Not all measurements have been performed in all experiments. 


\section{The current control strategy}

The current control system for fed-batch $B$. licheniformis fermentations uses an initial static feed ramp and then employs $D O$-controlled feed with minimum and maximum ramps. $D O$-controlled feed means that the feed is used as the control parameter to keep $D O$ at its setpoint (20\%).

Currently, control of the fermentations is done using the commercial distributed process control system DeltaV. Although this system is capable of many types of advanced control, only ordinary PI control is used here to control a set of process parameters separately (for instance $F, T, P$ and $\mathrm{pH}$ ).

The initial feed ramp and its split into minimum and maximum ramps is shown in figure 7.1 along with other preset ramps used in the process. This strategy is suitable for avoiding oxygen limitation, but there is no feedback system to avoid overflow metabolism as the $D O$ level can be high even though the system is at overflow, for instance at low biomass concentrations and a high oxygen transfer rate. The static ramp and the maximum limitation on the feed is what prevents too high feed levels at the start of the process, but these do not employ any sort of feedback. This means that variations in the growth of the biomass are not taken into consideration by the control system during the first hours of the fermentation.

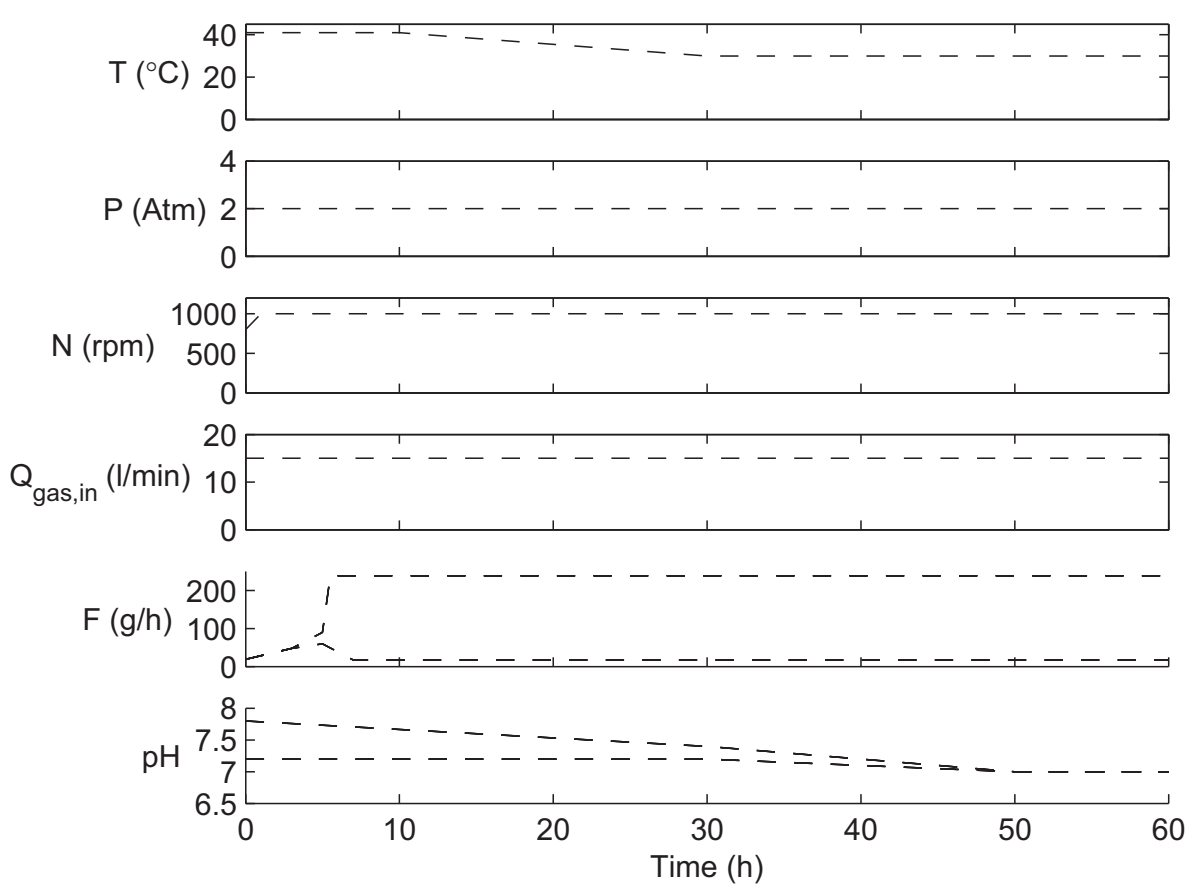

Figure 7.1 Ramps for temperature $(T)$, pressure $(P)$, agitator speed $(N)$, aeration rate $\left(Q_{\text {gas }, i n}\right)$, feed rate $(F)$ and $\mathrm{pH}$ using the currently existing controller in a 201 fermentation. After $60 \mathrm{~h}$ all displayed parameters are static. For feed rate and $\mathrm{pH}$, maximum and minimum ramps are shown.

In addition to these limitations in the control system, it can be troublesome to scale up a process based solely on a fixed feed ramp since mass transfer of oxygen to the broth varies significantly depending on reactor scale (Nielsen and Villadsen and Lidén 2003, p. 495). 
Agitator speed and aeration rate follow predefined ramps and are for the most time at their respective maxima, as shown in figure 7.1. Similarly, pressure is held constant throughout the fed-batch phase. These as well as other process parameters such as $\mathrm{pH}$ and temperature are controlled using separate PI-control loops in DeltaV. $\mathrm{pH}$ is controlled through addition of acid and base, when the current value goes outside the limits shown in figure 7.1.

\subsection{Reference experiment}

This experiment, controlled using the existing $D O$-regulated feed method, was performed in order to get data for comparisons with experiments performed using the probing control strategy. The experiment was performed in a 201 tank as no $2.5 \mathrm{~m}^{3}$ tank was available. An overview of the reference experiment can be seen in figure 7.2 , where the levels of product activity shown are normalised by the highest value measured in this experiment. Normalisation by the same value was done to product activity measurements from other experiments shown in this report, to allow comparison with the reference experiment.

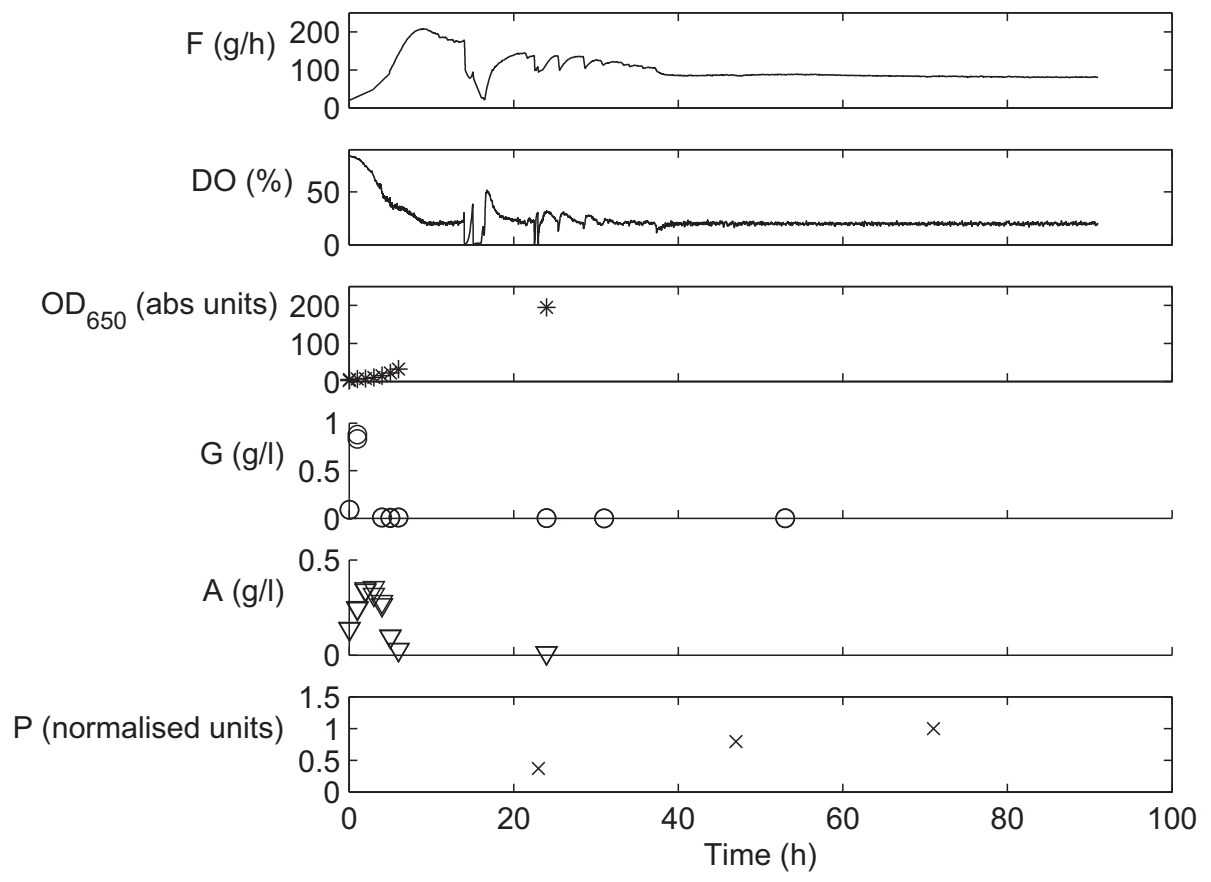

Figure 7.2 Overview of the reference experiment. Product activity levels are normalised by the highest measured value.

As seen in figure 7.2, an outgrowth phase took place during the first $9 \mathrm{~h}$ of the fermentation, reaching its end when $D O$ first went below its setpoint. After this $F$ was gradually decreased until a steady level was reached, which could be expected as $T$ and $\mathrm{pH}$ were changed at the same time to decrease the biomass growth rate.

At 3-5 h $F$ followed the maximum ramp, leading to a decrease in acetate levels. This indicates that the maximum ramp at $3-5 \mathrm{~h}$ is set sufficiently low that most of the acetate created at $1-3 \mathrm{~h}$ is consumed during this time. However, this may not be the case should the initial biomass concentration or the biomass growth rate for the 
first hours be lower than expected, as the maximum feed ramp does not employ any feedback.

At one point around $14 \mathrm{~h}$ the outlet filter of the bioreactor was clogged by foam from the fermentation, leading to $D O$ reaching zero for a while and $F$ thus being decreased by the controller. Eventually the filter was replaced and the process continued in the expected manner. This is not considered to have had any significant effects on the experiment, as after the problem was corrected $F$ returned to following the same approximately linearly decreasing trend, indicating that the event had little influence on the growth of biomass. It is possible that the production of the product would have decreased during this time, but as the problem only occurred for a rather short time any such effects should not be major. 


\section{Development experiments}

The controller and the setup of the process was developed in several steps, in which a prototype of the controller was used to control fermentations in 201 tanks after each of which the outcome was studied and changes to the controller and the experimental setup were performed. The product of the development experiments was a controller capable of controlling fermentations in $2.5 \mathrm{~m}^{3}$ tanks from start to end without manual intervention.

A short summary of the purposes of these development experiments can be seen in table 8.1. They are described in greater detail under their respective headlines.

\begin{tabular}{cll} 
Experiment & Purpose & Outcome \\
\hline 1,2 & $\begin{array}{l}\text { Characterisation of the process, first at- } \\
\text { tempts at probing control }\end{array}$ & $\begin{array}{l}\text { Data for process characterisation, } \\
\text { proof of concept }\end{array}$ \\
3 & Test of continuous feed pump action & Changed feed pump action \\
4 & Run a fermentation from start to end & $\begin{array}{l}\text { First indications of usefulness in actual } \\
\text { process }\end{array}$ \\
$5,6,7$ & $\begin{array}{l}\text { Implement a } D O \text { safety net, recalibrate } \\
\text { feed pump, test feed dynamics in large } \\
\text { scale }\end{array}$ & $\begin{array}{l}\text { Working safety net, decreased pulse } \\
\text { length, data for feed pump control in } \\
\text { large scale }\end{array}$ \\
& $\begin{array}{l}\text { First implementation of probing control in } \\
2.5 \mathrm{~m}^{3} \text { scale }\end{array}$ & $\begin{array}{l}\text { Modified probing control } \\
\end{array}$
\end{tabular}

Table 8.1 Summary of each experiment's purpose.

$D O$ control in all experiments used agitator speed and aeration rate as control parameters, with them starting at their respective minima and first controlling the agitator speed until it reached its maximum and then switching to control of the aeration rate. Should the aeration rate reach its minimum value the controller went back to controlling the agitator speed. Suitable gains for the $D O$ controller were first estimated based on knowledge of the process and then modified through studying controller behaviour in the experiments. No actual tuning using for instance the kappatau method was done as $D O$ controller behaviour was sufficiently good that probing pulses could be performed, so running additional experiments for tuning purposes did not have priority.

In the first two experiments, the $D O$ value at the end of a pulse was used to determine $D O_{\text {pulse }}$ as according to the theory, the peak of the $D O$ response should be found there. In further experiments, the highest/lowest $D O$ value during a pulse (depending on the direction of the pulse) was used to determine $D O_{\text {pulse }}$, as it appeared that the peak of the $D O$ response sometimes occurred before the end of a pulse due to disturbances. 


\subsection{First and second development experiments}

Implementation of probing control in B. licheniformis fermentations has never been carried out before. To evaluate the feasibility of the concept, process characterisation was therefore needed to determine whether overflow metabolism occurs in a similar manner as in E. coli fermentations, which probing control has been tested extensively on (Åkesson 1999; Velut 2005; de Máre 2006; Henes and Sonnleitner 2007). For a good visualisation of $E$. coli overflow metabolism, see (Xu and Jahic and Enfors 1999, p. 85).

In these experiments, an initial batch phase was used for characterisation of the process in regard to overflow metabolism. As the organism's rate of glucose consumption was unknown several different starting concentrations of glucose were used $(5,10$ and $20 \mathrm{~g} / \mathrm{l})$. The results of the first experiment allowed a fairly good estimation of the duration of the batch phase during the second experiment, meaning that one of the batches (PBFM75) in that experiment gave a good picture of process behaviour during and just after the batch phase. Figure 8.1 shows measured process data in batch PBFM75 during the batch phase. The batch phase ended when the glucose concentration reached zero shortly after $5 \mathrm{~h}$, where the exponential growth of bacteria stopped due to lack of the preferred substrate and acetate was consumed instead of glucose.
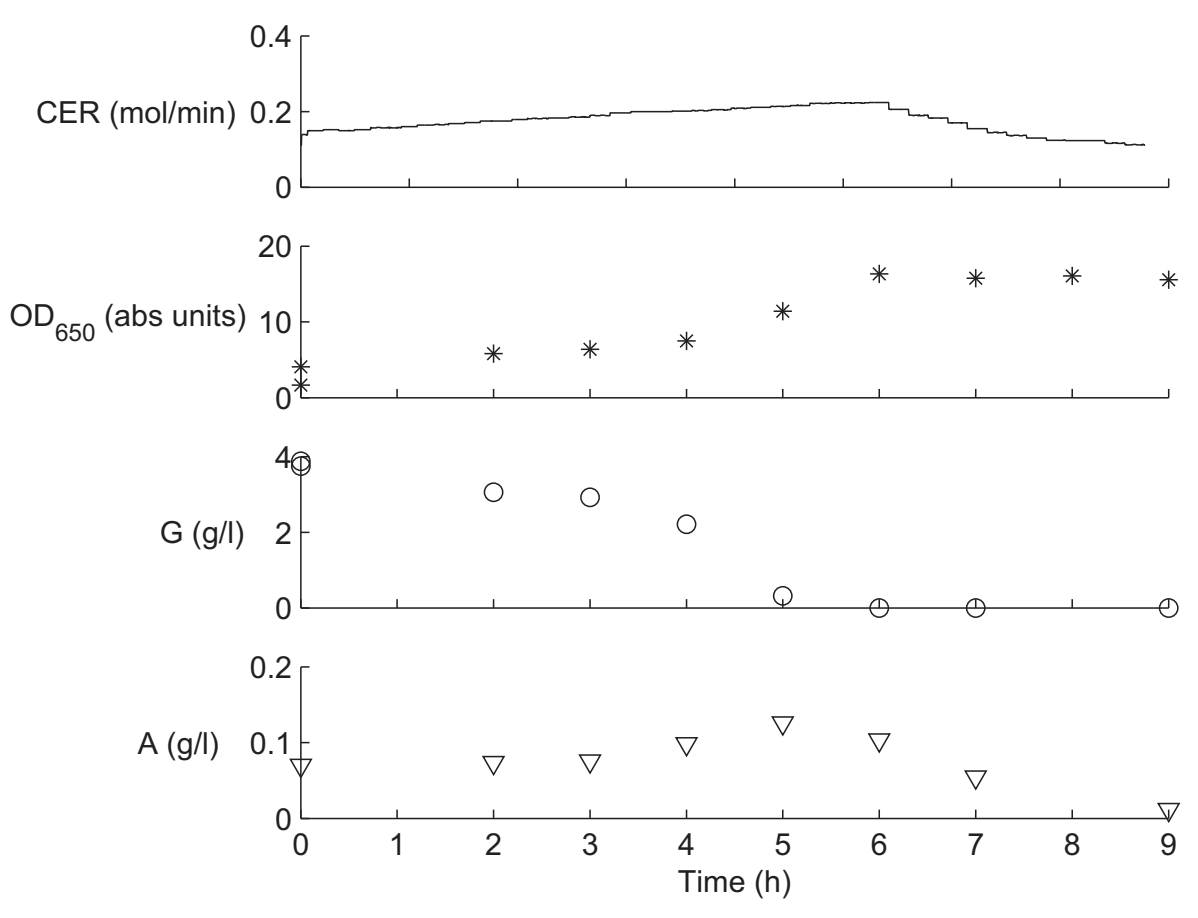

Figure 8.1 Process data during batch phase in batch PBFM75 during the second development experiment.

Based on these results, it appears that $B$. licheniformis qualitatively behaves similarly to $E$. coli in regard to overflow metabolism in that it produces acetate in the presence of high levels of glucose. It also behaves similarly in that the production of acetate as well as the exponential growth of biomass end and acetate is consumed instead as soon as the process becomes glucose-limited (just after $5 \mathrm{~h}$ into the process in the second experiment, as seen in figure 8.1). 
After the batch phase in the first experiment, a first test of the viability of probing control was performed. Using manual pulses, meaning that the feed pump was manually switched on (at its maximum) for three minutes at a time while $Q_{a i r, \text { in }}$ and $N$ were held constant, very distinct pulse responses could be seen in $D O$ as shown in figure 8.2. It should be noted that the mirrored $D O$ responses seen just after the initial $D O$ responses are due to the DeltaV feed rate controller decreasing $F$ temporarily to compensate for the manual pulses (see section 7), leading to a temporary increase in DO.

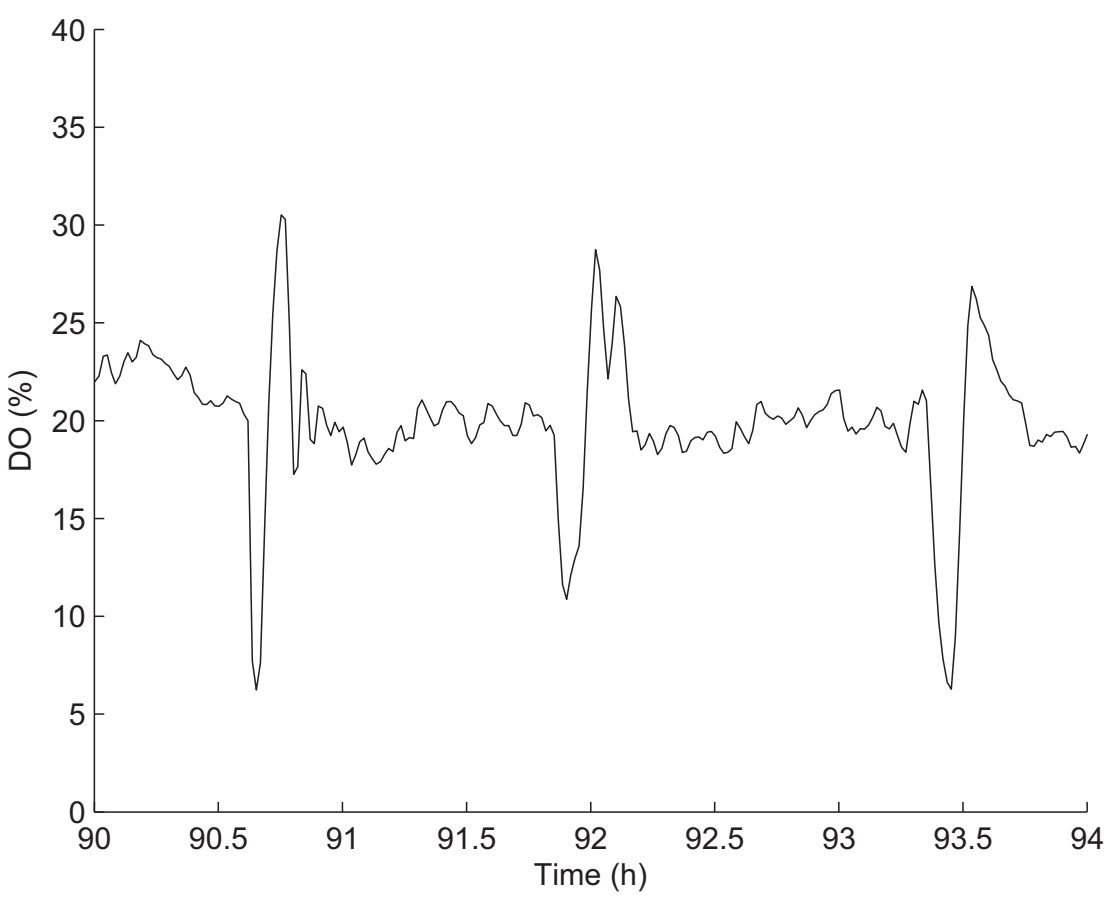

Figure 8.2 Responses to manual pulses in the first development experiment.

In the second experiment, the dynamics of the agitator speed and aeration rate were studied by performing setpoint steps, as illustrated in figure 8.3. Measurements in the graphs over the setpoints and process values led to the conclusion that both systems behaved like approximately linear first-order systems with time constants of about 5 seconds for the aeration rate and about 50 seconds for the agitator speed. This indicates that $D O$ control can be performed much faster when using the aeration rate as the control parameter than when using the agitator speed.

These first two experiments gave clear indications that probing control could be implemented in the process in question, as its overflow metabolism mechanisms appeared to be similar to those of $E$. coli processes and the dynamics of the process and of the $D O$ electrode were fast enough that clear responses to feed pulses in the minute range could be detected when studying $D O$ curves visually. It was therefore possible to move on to improving the process and running fermentations entirely controlled by the probing controller. 

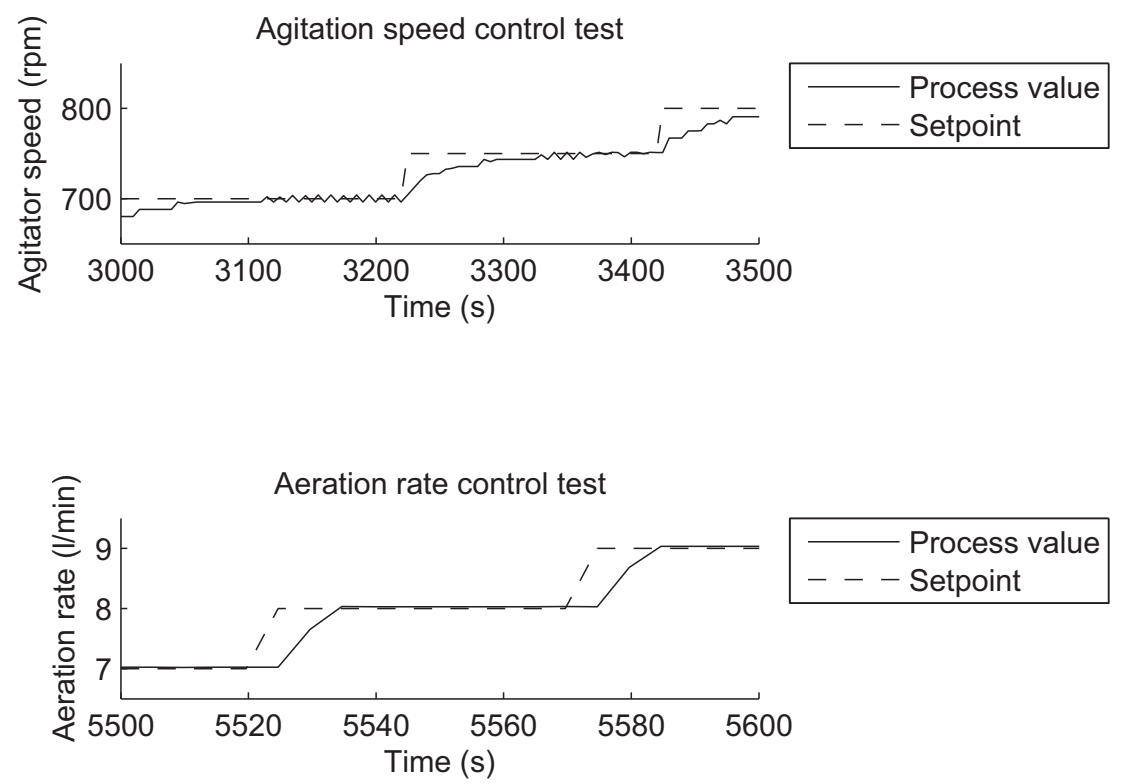

Figure 8.3 Study of agitator speed and aeration rate dynamics in the second development experiment. This illustration shows only small parts of the ranges within which the dynamics were studied.

\subsection{Third development experiment}

In this experiment the feed dynamics were optimised as the previously existing feed rate control strategy (as described in section 7) was not designed for swift changes in $F$, which presumably led to more spread out, less defined pulses. The pulse-wise addition of feed also caused $D O$ noise, which is undesirable as it means a higher value of $D O_{\text {reac }}$ is required (see section 5.2). A more desirable method for controlling the feed rate would be to vary the feed pump speed to achieve different values of $F$ without having an oscillating $F_{p v}$, with the feed pump speed controlled directly by the probing controller. Such a feed strategy was therefore implemented in this experiment.

The electrical system controlling the feed pump for one tank (109M) was rebuilt and the controller changed accordingly, to allow continuous feed addition by running the pump constantly at speeds correlating to the current $F_{s p}$ rather than writing $F_{s p}$ to DeltaV and letting its feed regulator control the pump. Calibration of the feed pump was done according to its specifications. Although the probing controller could only communicate with the process via DeltaV, it was possible to send the signal through DeltaV without modification and thus use voltage values corresponding to different pump outputs as the control parameter in the probing controller. This gave clear improvements in $D O$ behaviour as the $D O$ response to a pulse became more distinguishable from the baseline, as shown in figure 8.4, meaning that $D O_{\text {reac }}$ can be decreased. In experiment 3 pulses had a somewhat lower amplitude, but while the amplitude of the $D O$ responses became lower they were still easily distinguishable from the fairly straight baseline as the $D O$ responses to pulses were more defined and less spread out in time, indicating that the assumption regarding pulses becoming 
more defined was correct.
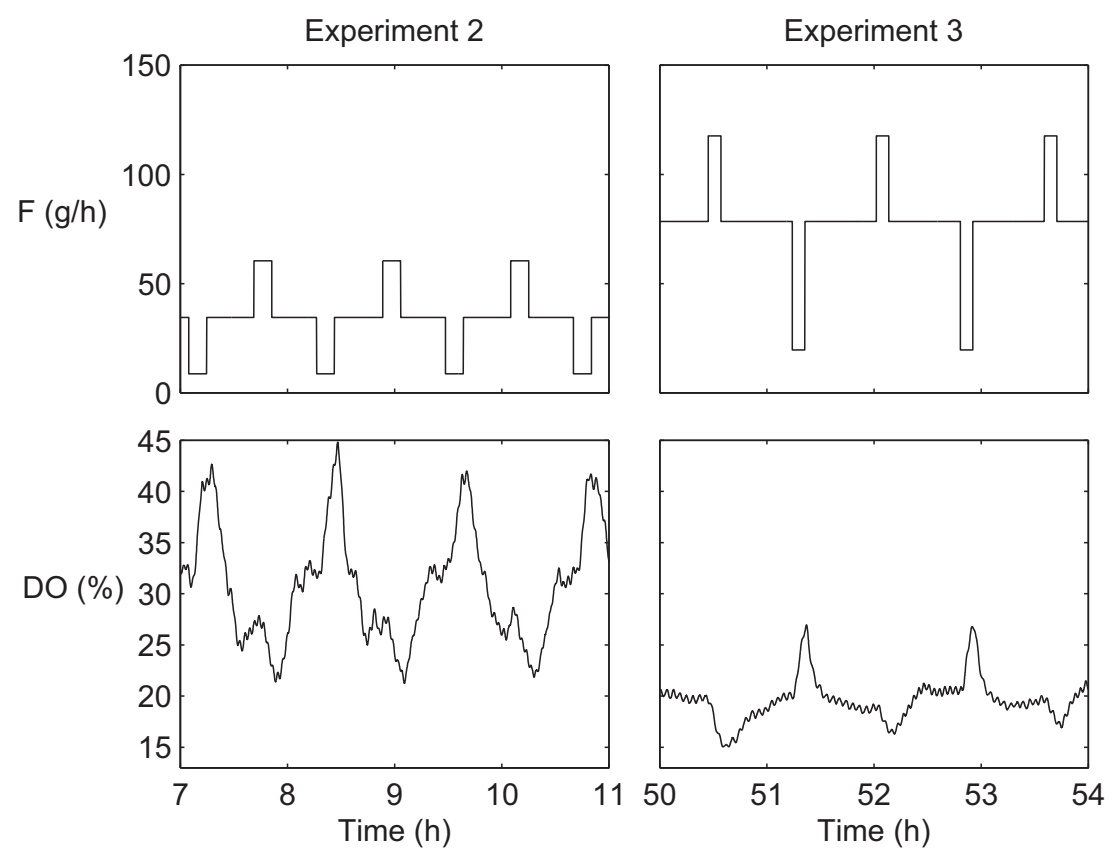

Figure 8.4 Comparison between DO in tank 109M during the second and third development experiments.

A distinct oscillatory pattern in $D O$ could be noticed (see figures 8.4 and 8.5), at the time of this experiment it could be concluded that this was not due to bad $D O$ control but the actual reason was unknown. Such oscillations were undesirable as without them, the $D O$ baseline could be expected to be considerably less noisy and it would be possible to decrease $D O_{\text {reac }}$ further and thereby improving the probing controller's performance.

Based on the outcome of this experiment, it was clear that pulse performance was significantly improved with the continuous feed method and it was therefore used in one fermentor during all small-scale experiments from this point. All data from 201 fermentations in this report from this point on is from this fermentor. It was not possible to use this method in $2.5 \mathrm{~m}^{3}$ experiments as a different feed method is used there and can not be easily changed as it would require rebuilding the system physically and installing new machinery, but this feed method was expected to cause considerably fewer problems than the non-continuous feed method in the 201 fermentors. This is because in the $2.5 \mathrm{~m}^{3}$ tanks $F_{p v}$ is measured directly by a flow meter and used to regulate the feed rate, meaning that feed rate control should be more suited to fast changes in $F_{s p}$ than in the 201 tanks.

After the experiment, data from the controller was compared to the actual feed volume used during the experiment and the control of the feed pump was recalibrated based on these data. 


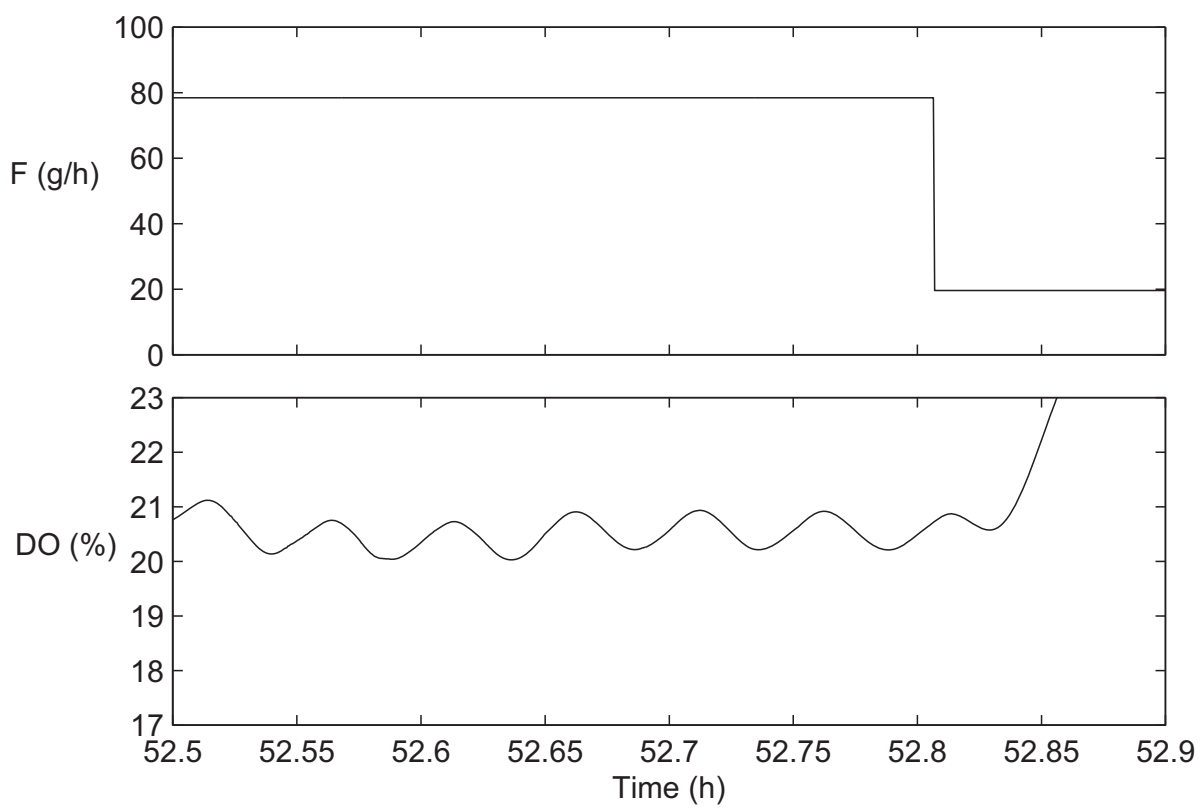

Figure 8.5 Oscillations around the $D O$ baseline during the third experiment (see figure 8.4 for $F$ during the timespan in this figure).

\subsection{Fourth development experiment}

After the viability of probing control in the process had been tested and the feed method improved to achieve more well-defined pulses, the next step was to run a fermentation controlled by the probing controller from start to end. In the very first hours of the fermentation a static feed ramp had to be employed however, to allow the biomass to grow enough that the setpoint for the $D O$ controller $(20 \%)$ could be reached before starting $D O$ control and probing control. This was necessary as even with $N$ and $Q_{g a s, \text { in }}$ at their respective minima at the start of the process, $D O$ was considerably higher than its setpoint because of the very low rate of oxygen consumption in the system at this time. Suitable values for the static feed ramp were taken from the static ramp used at the start of fermentations utilising the existing control strategy (see section 7.1), however in this fermentation the ramp would be stopped and probing control started as soon as the $D O$ setpoint was reached.

Figure 8.6 shows the most important data from fermentation PBFM80 in the fourth experiment. As seen there the controller was capable of detecting $D O$ responses and steadily increasing $F$, but the increment appeared to have occurred rather slowly compared to the reference experiment. This may be due to $t_{c, \min }$ being set as high as $30 \mathrm{~min}$, meaning that the $F$ baseline could only be altered once every $40 \mathrm{~min}$ at most, which may have imparted unnecessarily harsh restrictions on $\mu$. Controller parameters during the experiment can be seen in table 8.2.

Due to programming errors there were problems in the controller at certain points during the fourth experiment, but to some extent the outcome of these could be used to study the controller's robustness characteristics qualitatively. At $5 \mathrm{~h}$ un-updated program code caused undesired changes in $F$, at $29 \mathrm{~h}$ the controller program crashed due to a mistake when making changes to it and when it was restarted $F$ was con- 

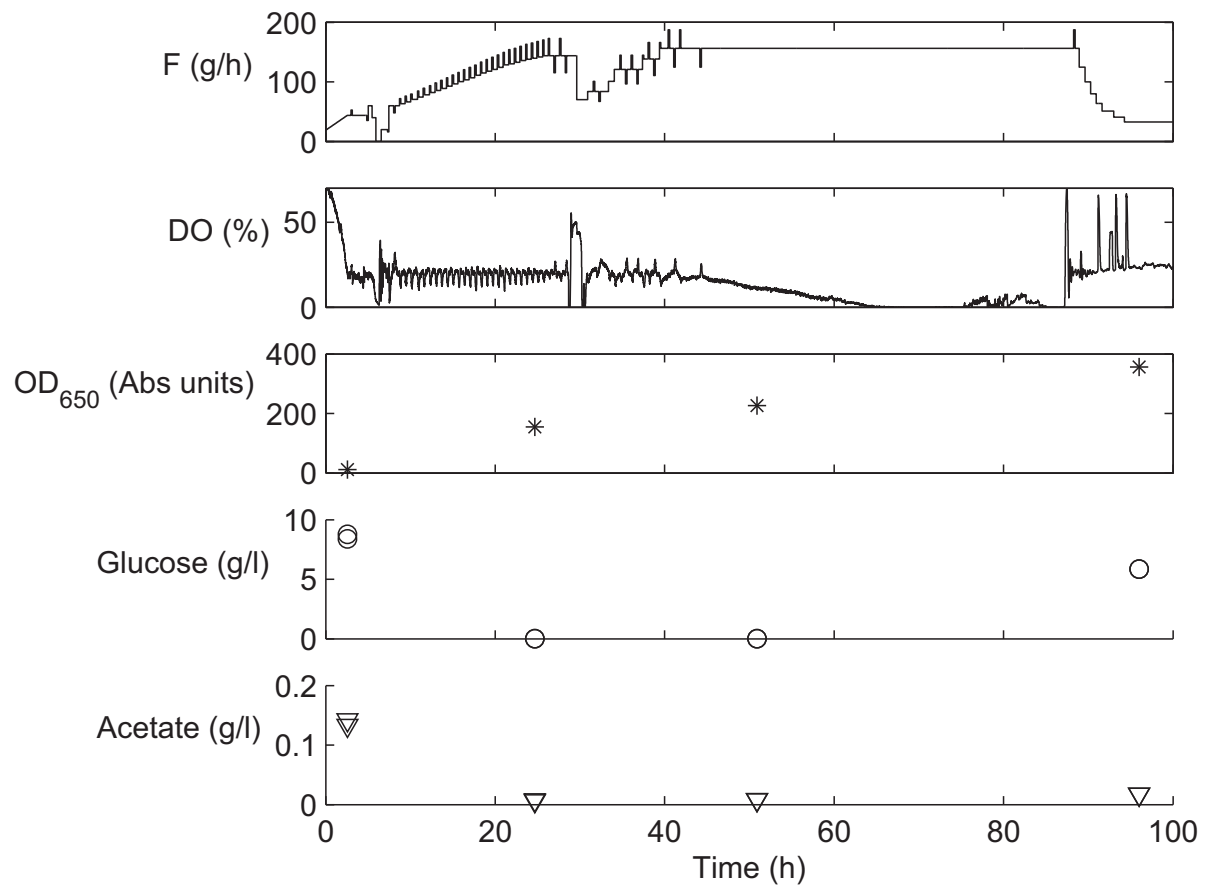

Figure 8.6 Overview of fermentation PBFM80 in the fourth development experiment.

siderably lower than before the control system crash. However, the controller slowly returned $F$ to a value in the vicinity of that before the crash, indicating that the robustness of the controller is sufficient to overcome such events.

Towards the end of the experiment the batch reached oxygen limitation as it was no longer possible to reach $D O_{s p}$ due to the maximum limits of agitator speed and aeration rate being reached, meaning that probing control could not decrease $F$ since $D O_{p v}$ is considerably lower than $D O_{s p}$ and therefore the $D O$ stability criterium for performing new pulses is not fulfilled (see section 5.2). These limitations in the oxygen transfer capacity of the reactor then led to oxygen limitation in the system, as seen in the $D O$ trend in figure 8.6, which eventually caused the system to crash at $87 \mathrm{~h}$ due to oxygen limitation over a long period. Although the $O D_{650}$ measurement at $96 \mathrm{~h}$ (after the crash) gave a higher value than at $50 \mathrm{~h}$, this is reasonable as cells which have recently died can be expected to contribute somewhat to $O D_{650}$.

After this process crash it became possible for $D O$ to reach its setpoint, as a large part of the biomass had died in the crash so the oxygen consumption rate had decreased, meaning that new pulses could be performed. These pulses rapidly decreased $F$ to its minimum level $(30 \mathrm{~g} / \mathrm{h})$, which was coherent with the accumulation of glucose which could be seen at $96 \mathrm{~h}$ and thus showed that the controller is able to detect overflow metabolism and decrease $F$ to avoid it. The glucose was presumably accumulated after the crash, when the decreased biomass concentration meant that the glucose consumption would have decreased drastically. Despite the high concentration of glucose the acetate concentration was not particularly high at this point, which is explained by the low biomass concentration leading to a low production of acetate despite overflow metabolism taking place in the system.

During this experiment, a comparison of $O D_{650}$ and dry weight concentration was made, to verify that $O D_{650}$ was a good measure of biomass concentration. As seen in figure 8.7 the relationship between these is fairly linear, indicating that $O D_{650}$ can be 


\begin{tabular}{ll}
\hline Parameter & Value \\
\hline$t_{c y c l e}$ & $2 \mathrm{~s}$ \\
\hline$D O$ control & \\
\hline Agitation control & \\
$K_{c}$ & $400-1000 \mathrm{rpm}: 0.67$ \\
$T_{i}$ & $400-1000 \mathrm{rpm}: 0.4$ \\
\hline Aeration control & \\
$K_{c}$ & 0.01 \\
$T_{i}$ & 0.4 \\
\hline$F$ control & \\
\hline Feed ramp & \\
interpolation points & \\
$0 \mathrm{~h}$ & $18.7 \mathrm{~g} / \mathrm{h}$ \\
$3 \mathrm{~h}$ & $48 \mathrm{~g} / \mathrm{h}$ \\
$5 \mathrm{~h}$ & $75 \mathrm{~g} / \mathrm{h}$ \\
\hline Probing controller & \\
parameters & \\
$D O_{s p}$ & $20 \%$ \\
$t_{p}$ & $10 \mathrm{~min}$ \\
$t_{O}$ & $0 \mathrm{~min}$ \\
$t_{c, \text { min }}$ & $30 \mathrm{~min}$ \\
$D O_{\text {reac }}$ & $4 \%$ \\
$\gamma_{p}$ & 0.2 \\
$\kappa$ & 1 \\
\hline
\end{tabular}

Table 8.2 Controller parameters in the fourth development experiment.

used for comparing biomass concentrations. It should be noted that substances other than the biomass might also contribute to the optical density and this factor is not taken into consideration here. Ideally, a part of each sample used for measurement of $O D_{650}$ should be sterile filtered and the solution used as zero reference, to minimise the influence of other substances.

A feed pump calibration curve was created in this experiment, to make a more detailed calibration than before using several different values of $F$. To calculate $F_{p v}$ for different values of $F_{s p}$, the remaining feed weight was heavily filtered and the change in it over $20 \mathrm{~min}$ at a constant $F_{s p}$ was measured. As seen in figure 8.8 the calibration used in this experiment was fairly good, with the points in the calibration curve being in the vicinity of a linear trend with a slope of 1, although the slope decreases for higher values of $F_{s p}$.

The outcome of this experiment indicated that probing control could be used to control a $B$. licheniformis fed-batch fermentation, both reacting to overflow metabolism and to unnecessarily low values of $F$, finding a suitable level for $F$ and keeping it there. This was an important result as it was a strong indication that probing control was viable in the process, beyond what had been seen in the first and second development experiment. The crash at the end of the process indicated that to avoid oxygen starvation, the controller should be able to decrease $F$ when $D O$ becomes too low; a safety net for $D O$. 


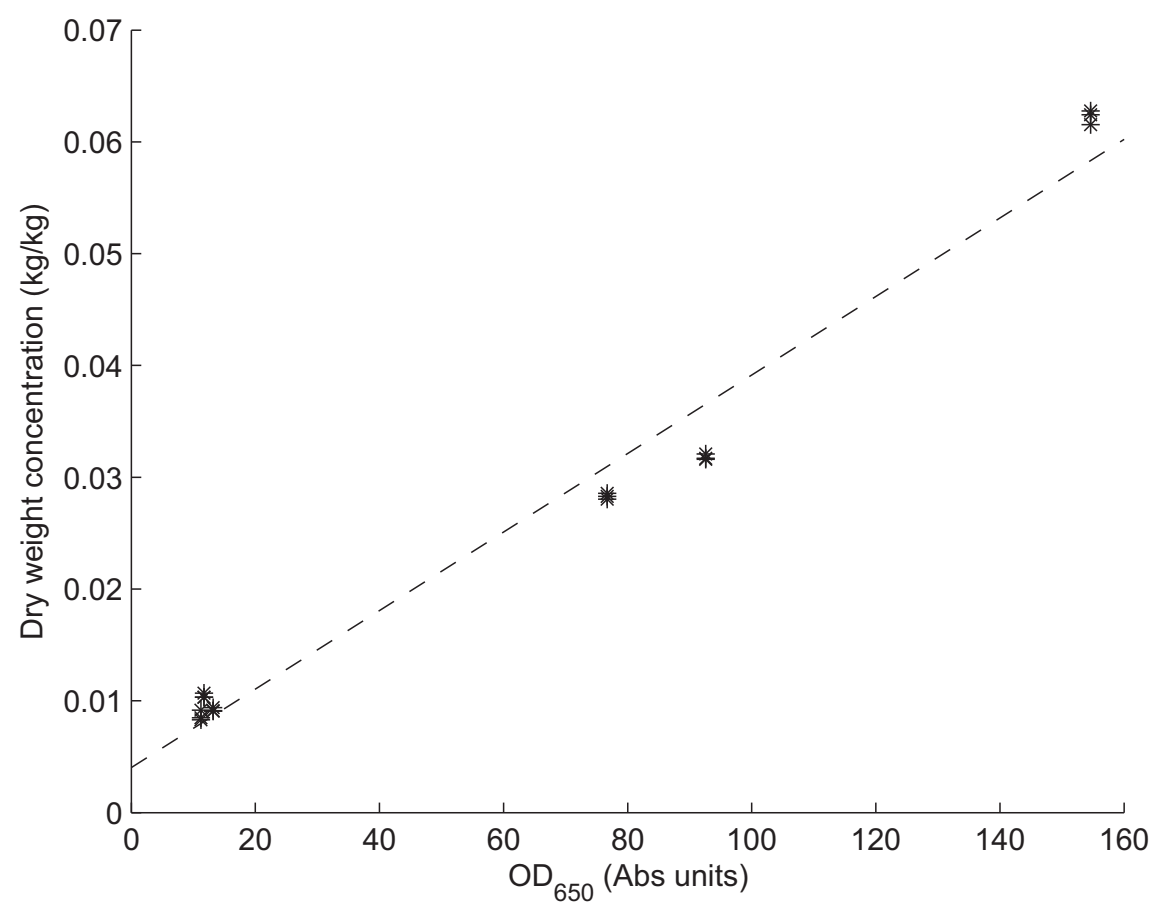

Figure 8.7 Comparison of $O D_{650}$ and dry weight concentration in the fourth development experiment, including a linear trend fitted to data.

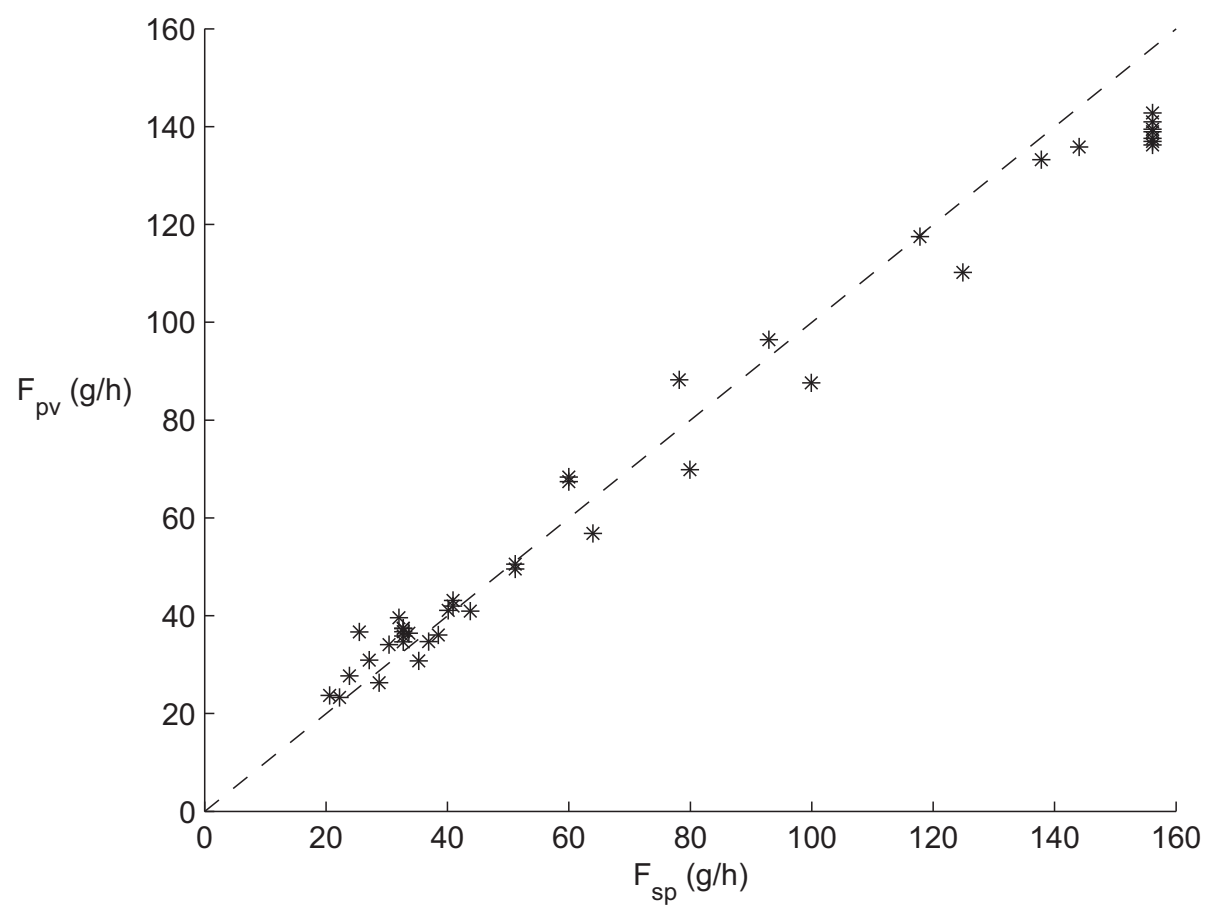

Figure 8.8 A calibration curve for the feed pump using data from the fourth development experiment, including a linear trend with slope 1 for comparison. 


\subsection{Fifth, sixth and seventh development experiments}

These experiments were all performed with the same aim as the fourth experiment; to run a fermentation from start to stop utilising the probing controller. The sixth experiment also allowed a first test of the feed rate dynamics in a $2.5 \mathrm{~m}^{3}$ tank. A $D O$ safety net was also added, which would decrease $F$ when $D O$ was too low and agitator speed and aeration rate had been at their respective maximums for a certain amount of time, to avoid a process crash such as that in the fourth development experiments. Such decrements would only take place after a set time $(15 \mathrm{~min})$ had passed since the last decrement or pulse, to avoid immediate, consecutive decrements and influence from pulse responses.

Gain scheduling for $D O$ control was implemented in the fifth experiment when using agitator speed as the control parameter, using different gains in two different agitator speed ranges, similar to what has been done in (Åkesson 1999, Paper III). In previous experiments the same gain had been used at all agitator speeds, leading to sluggish control at high speeds, although this was sometimes mitigated by changing the gain manually. The gain scheduling performed rather well although the gains were still changed manually during the experiment to improve performance, indicating that a greater number of ranges with different gains would improve $D O$ control.

The problem with oscillatory $D O$ behaviour which had first been noticed in the third development experiment was solved during the fifth development experiment, as it could be seen that this was due to bad pressure control in DeltaV. It could be corrected at $4.1 \mathrm{~h}$ by increasing the gain of the pressure controller; this gives a significant reduction of $D O$ noise as seen in figure 8.9.
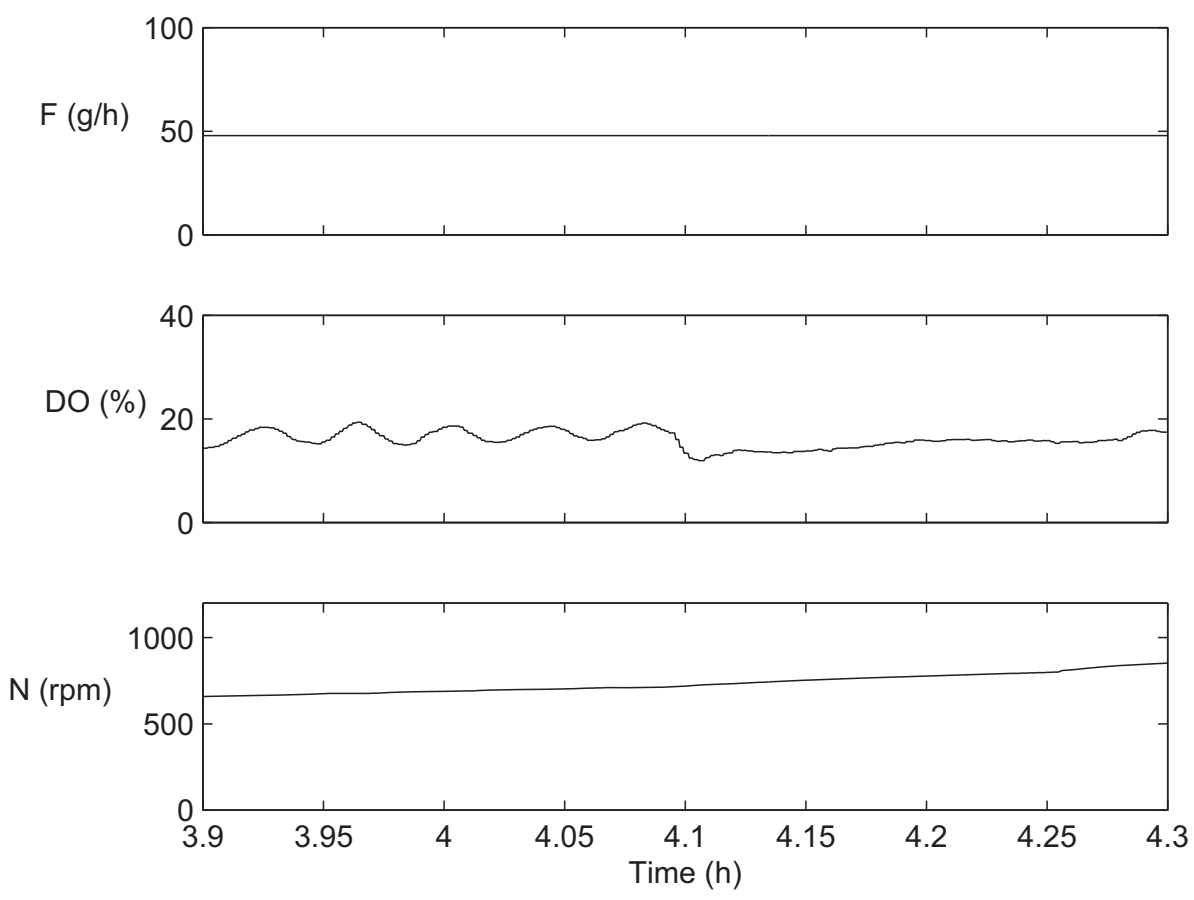

Figure 8.9 Removal of $D O$ oscillations as pressure control is improved in the fifth development experiment.

The sixth experiment was a direct repeat of the fifth, due to equipment failures in the fifth experiment. However, it was inoculated directly from a shake flask rather 
than from an inoculation tank, which was supposed to give approximately the same initial biomass concentration as when using an inoculaton tank but it turned out to actually be far lower than that. Due to the low inoculation volume the outgrowth phase was very long and it took a long time for $D O$ to reach its setpoint, meaning that the experiment could not be surveyed and samples taken while the process was in the outgrowth phase.

The seventh experiment was a second repeat of the fifth experiment and led to some new conclusions regarding $D O$ responses to pulses. As seen in figure 8.10, the pulses during the first $5 \mathrm{~h}$ appeared to give responses even though glucose was present and acetate was produced, indicating overflow metabolism.
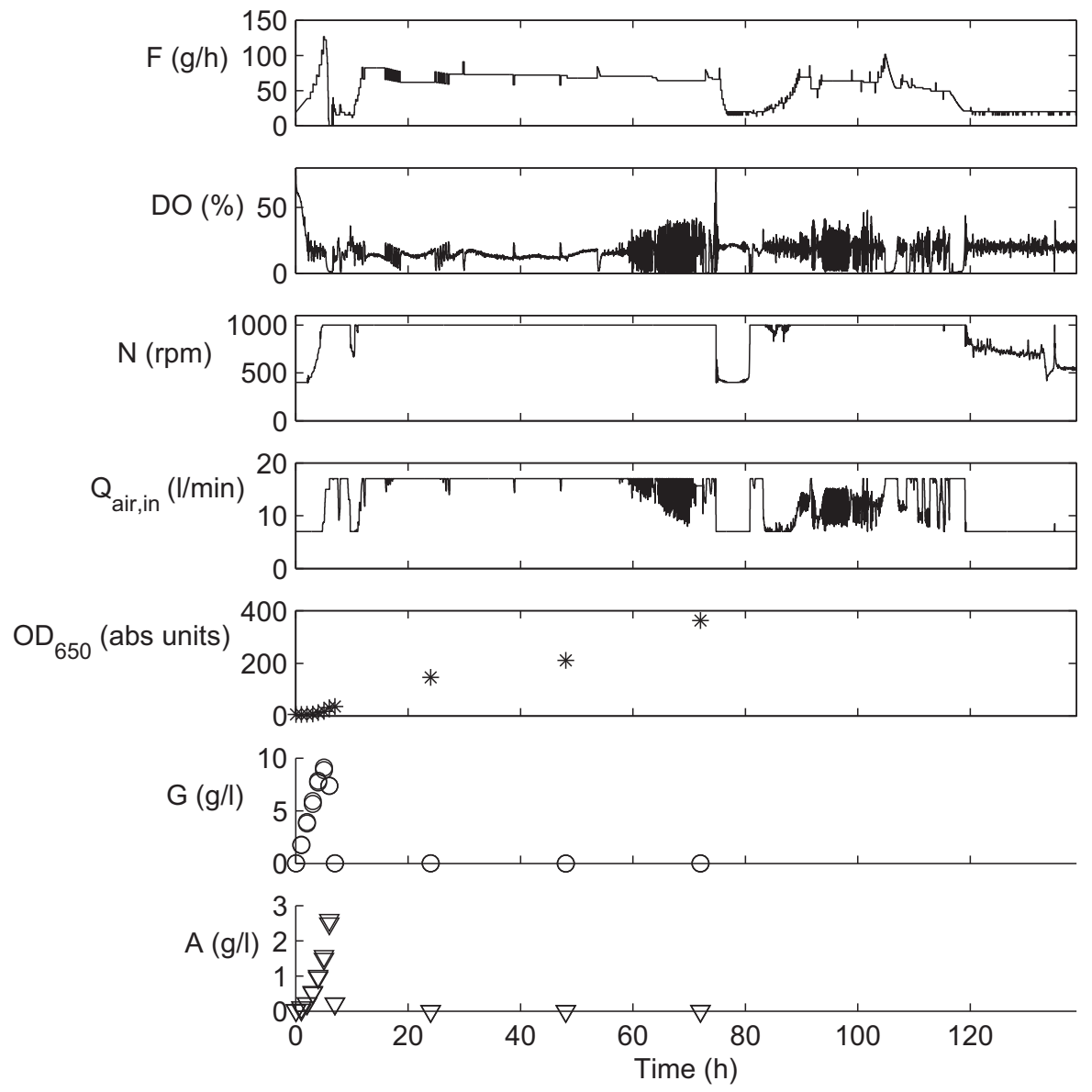

Figure 8.10 Overview of the seventh development experiment.

Following the theory behind probing control, $D O$ responses should not occur under such conditions. The conclusion is that if excess glucose is present and the biomass is thus growing at $\mu_{\max }$, up pulses with a $t_{p}$ of $10 \mathrm{~min}$ lead to a sufficient increase in biomass concentration that the additional respiratory capacity in the system significantly increases the oxygen consumption rate during the pulse. As the transfer of oxygen to the system is constant due to the paused $D O$ control during pulses (giving constant values of $N$ and $\left.Q_{\text {air,in }}\right), D O$ is decreased enough through this effect to register as a response to the pulse. This gives positive feedback in the feed regulation loop and $F$ will thus be increased until its maximum value is reached. For this reason, 
it is absolutely necessary to use lower values of $t_{p}$ than $10 \mathrm{~min}$.

At $47 \mathrm{~h}$ in the seventh experiment $t_{p}$ was set to $3 \mathrm{~min}$. Pulse responses were then somewhat faster than the pseudo-responses seen at the start of the experiment, so it was assumed that these were true pulse responses. A comparison between pulse responses at the start of the experiment and after the decrease in pulse duration can be seen in figure 8.11. It was concluded that pulses must not be too long, in order to avoid the pseudo-response effect seen here.
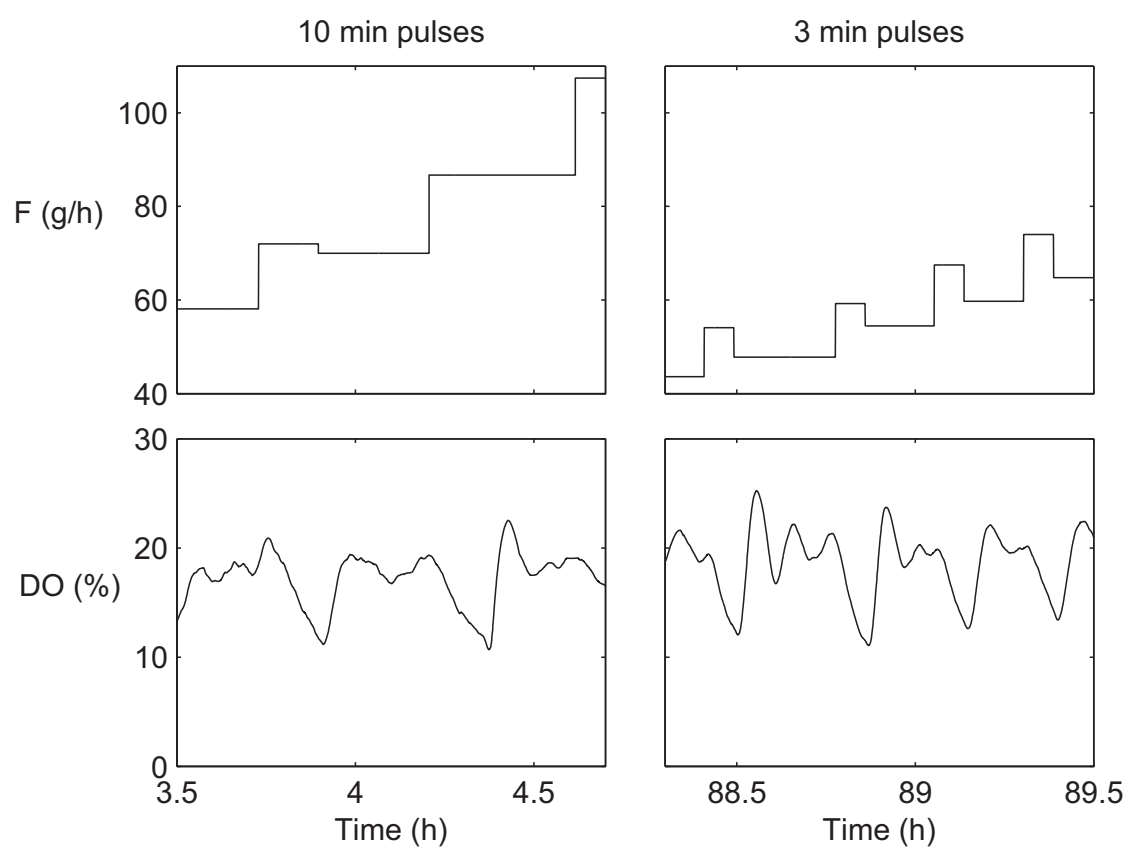

Figure 8.11 A comparison between pseudo-responses (left) and actual pulse responses (right) in the seventh experiment.

Recalibration of the feed pump was done before the fifth and the seventh experiment, based on calibration curves from previous experiments (see figure 8.8 for an example of such calibration curves), to achieve a $F_{p v}$ close to $F_{s p}$ at all levels. However after the seventh experiment it became clear that the pump gave an $F_{p v}$ which was approximately $100 \%$ too high for all $F_{s p}$ in that experiment, due to a miscalculation when calibrating the pump, which led to the accumulation of glucose and acetate during the static feed ramp in that experiment.

The safety net acted as expected during the later part of the seventh experiment, although it was decided that the decrements should be considerably smaller and with a shorter minimum time between them, to find a suitable feed level and thus avoid the oscillations in $F$ seen in figure 8.10 between 15 and 30 h, caused by consecutive decrements and pulses.

The gain scheduling used in the seventh development experiment appeared to improve $D O$ controller behaviour, although it was believed that adding more bands with different gains may improve it further. The gains used are shown in table 8.3.

As seen in figure 8.10,DO showed a highly oscillatory behaviour at several points after $60 \mathrm{~h}$ into the fermentation. This is because of excessive foaming in the system.

The feed dynamics in the $2.5 \mathrm{~m}^{3}$ tank, when tested in the sixth experiment, showed an oscillatory pattern as could be expected due to the non-continuous feed 


\begin{tabular}{lll}
\hline$N(\mathrm{rpm})$ & $K_{c}$ & $T_{i}$ \\
\hline $400-800$ & 1 & 0.4 \\
$800-1000$ & 3 & 0.4 \\
\hline
\end{tabular}

Table 8.3 Gain scheduling parameters for the PI controller for $D O$ when regulating the agitator speed in the seventh development experiment.

method (see section 6.1 for details). Similar to fermentations in 201 tanks, a steady $F_{p v}$ with as small oscillations as possible is desirable as described in section 8.2. At first the system was set to use a set shot volume, varying the time between shots to achieve different values of $F$, which can keep the amplitude of the oscillations low within a certain interval of $F_{s p}$ but low values of $F_{s p}$ lead to longer times between shots and larger oscillations as seen in figure 8.12 when $F_{s p}$ was decreased from $5 \mathrm{l} / \mathrm{h}$ to $1 \mathrm{l} / \mathrm{h}$ (the minimum value of $F$ ) at $1.7 \mathrm{~h}$.

To minimise oscillations in $F$ the system was instead set to vary the shot volume and use a fixed time between shots at $2.6 \mathrm{~h}$, with this time being set to its minimum $(30 \mathrm{~s})$, which gave good results even at the minimum $F$. However, as seen in figure 8.12 , using the minimum time between shots meant that $F_{p v}$ reached a maximum around $12 \mathrm{l} / \mathrm{h}$. This indicated a need to vary the time between feed shots during an experiment to achieve good feed dynamics at all feed rates, which was made possible through adding a feature for this in DeltaV and modifying the probing controller accordingly.

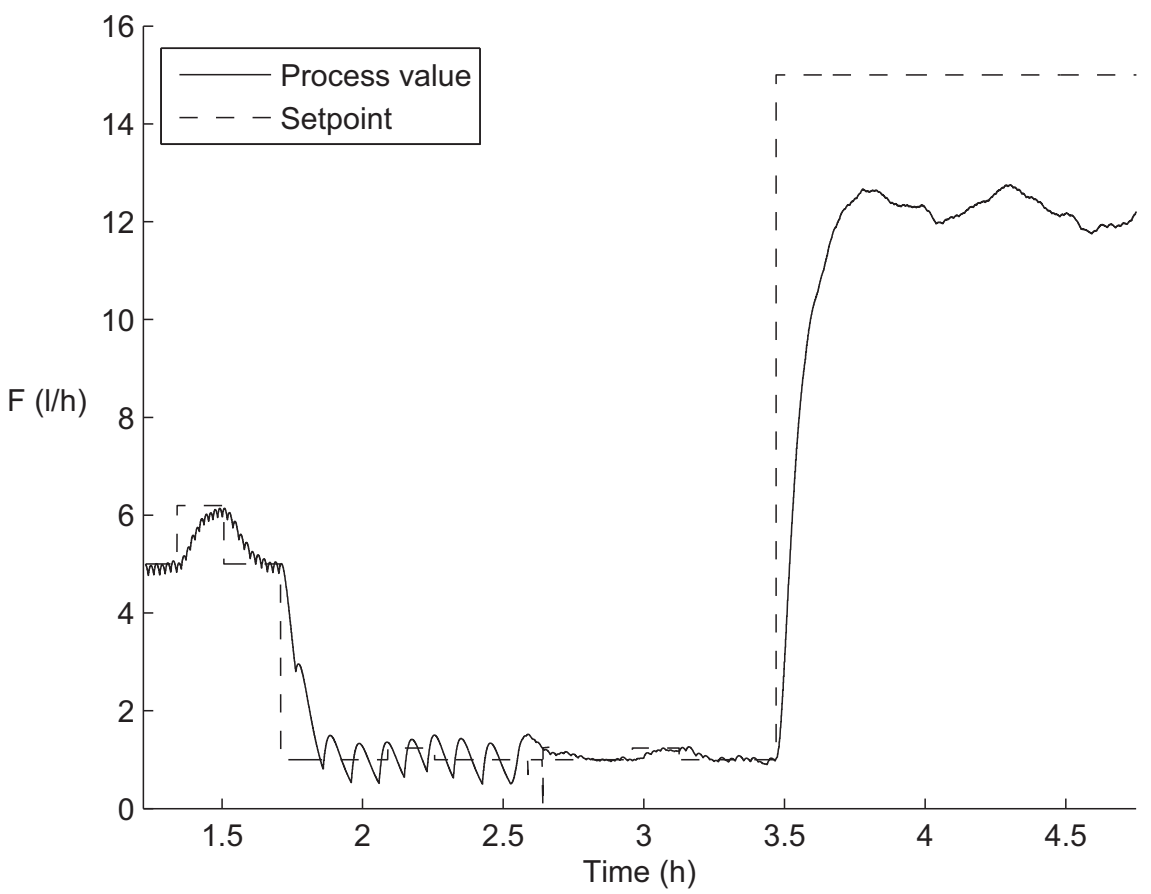

Figure 8.12 Test of feed dynamics in $2.5 \mathrm{~m}^{3}$ tank during the sixth experiment. 


\subsection{Eighth development experiment}

The first seven development experiments had all been performed in 201 fermentors, but it was desirable to see how well the probing controller could control the process in larger scale and if there would be any scale-up issues. At this point the concept was considered proven enough that a fermentation in larger scale could be performed and it was therefore decided to run the process in $2.5 \mathrm{~m}^{3}$ scale controlled by the probing controller in the eighth development experiment. Although no 201 fermentation had run perfectly at this stage, the seventh development experiment showed good controller behaviour after the pulse time had been decreased to 3 minutes.

The controller was largely unchanged between the seventh and eighth development experiment, although all controller gains and the initial static feed ramp were rescaled for $2.5 \mathrm{~m}^{3}$ fermentations. The parameters used for the controller at the start of the experiment are shown in table 8.4.

\begin{tabular}{ll}
\hline Parameter & Value \\
\hline$t_{\text {cycle }}$ & $2 \mathrm{~s}$ \\
\hline$D O$ control & \\
\hline Agitation control & \\
$K_{c}$ & $100-140 \mathrm{rpm}: 0.167$ \\
& $140-185 \mathrm{rpm}: 0.333$ \\
& $185-215 \mathrm{rpm}: 0.667$ \\
& $215-275 \mathrm{rpm}: 1$ \\
$T_{i}$ & $100-275 \mathrm{rpm}: 0.4$ \\
\hline Aeration control & \\
$K_{c}$ & 5 \\
$T_{i}$ & 0.4 \\
\hline$F$ control & \\
\hline Pump pause time & $0-10 \mathrm{l} / \mathrm{h}: 30 \mathrm{~s}$ \\
& $10-19 \mathrm{l} / \mathrm{h}: 60 \mathrm{~s}$ \\
\hline
\end{tabular}

\begin{tabular}{ll}
\hline Feed ramp & \\
interpolation points & \\
$0 \mathrm{~h}$ & $0.6 \mathrm{l} / \mathrm{h}$ \\
$3 \mathrm{~h}$ & $1.5 \mathrm{l} / \mathrm{h}$ \\
$5 \mathrm{~h}$ & $2.9 \mathrm{l} / \mathrm{h}$ \\
\hline Probing controller & \\
parameters & \\
$D O_{s p}$ & $20 \%$ \\
$t_{p}$ & $3 \mathrm{~min}$ \\
$t_{o}$ & $0 \mathrm{~min}$ \\
$t_{c, \text { min }}$ & $5 \mathrm{~min}$ \\
$D O_{\text {reac }}$ & $4 \%$ \\
$\gamma_{p}$ & 0.258 \\
$\kappa$ & 1 \\
\hline
\end{tabular}

Table 8.4 Controller parameters at the start of the eighth development experiment.

The initial static ramp showed difficulties in decreasing the $D O$ to the setpoint at $20 \%$, so $D O_{s p}$ was changed to $25 \%$ to allow probing control to start sooner, meaning that probing control could start after $6 \mathrm{~h}$. An overview of the experiment is provided in figure 8.13 . 

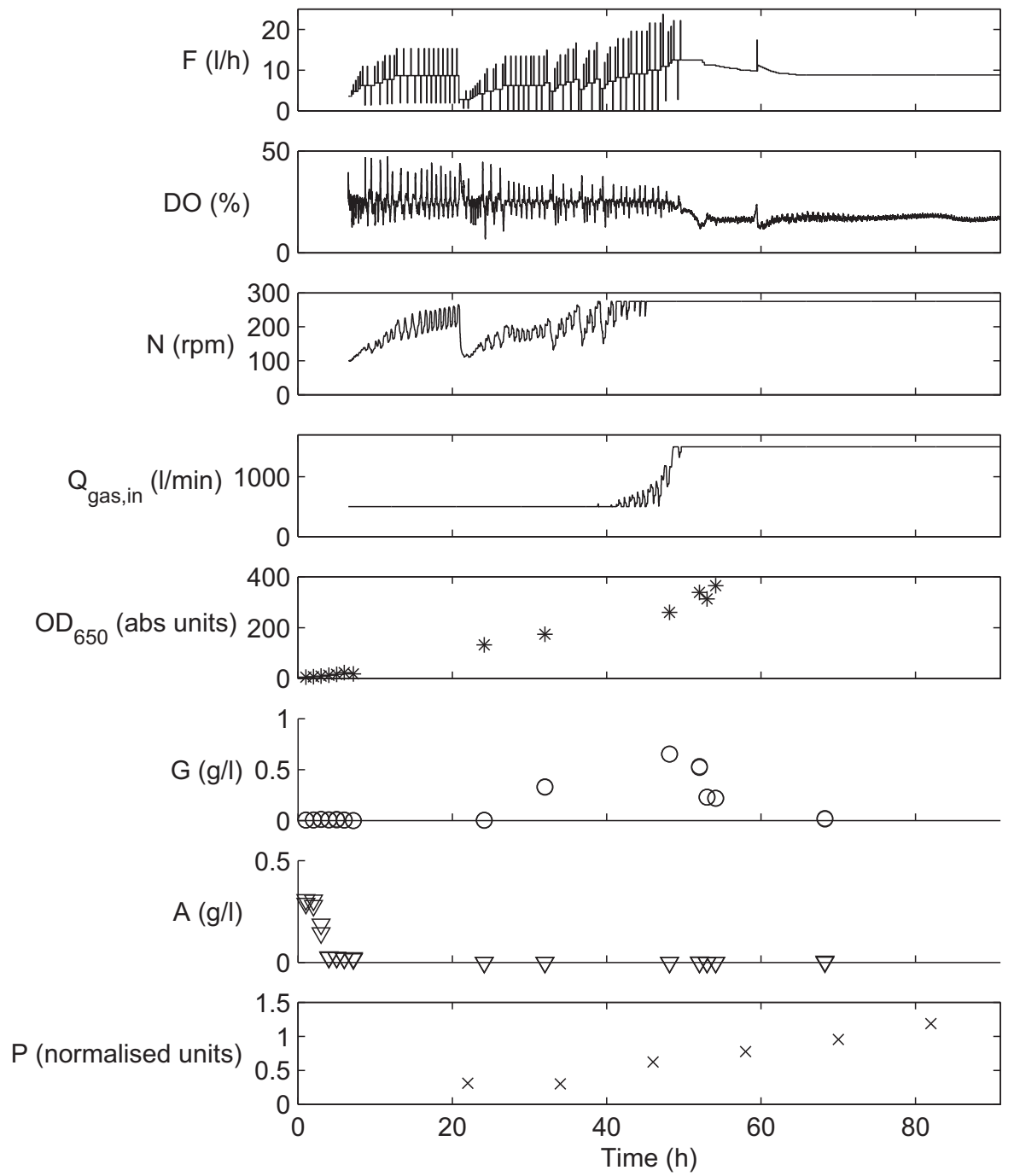

Figure 8.13 Overview of the eighth development experiment. A data handling error led to the data measured online for the first $6.5 \mathrm{~h}$ not being saved. However, this time period mainly includes the static feed ramp.

It was noted that due to the slow feed dynamics, the change in $F_{p v}$ during a pulse was only about a third of $F_{p u l s e}$ and the peak of $F_{p v}$ (and thus, theoretically, the maximum of $D O_{\text {pulse }}$ ) occured after the pulse was over (see figure 8.14). To compensate for this $F_{\text {pulse }}$ was increased to three times its starting value. This meant that $\Delta F_{\max }$ was increased equally much and that $F_{s p}$ became zero during down pulses (as seen in figure 8.13). Despite the increase in pulse amplitude, at $14.5 \mathrm{~h} F$ was heavily decreased as the $D O$ response to a pulse occurred after the pulse was over and was therefore not registered by the controller and the increased $\Delta F_{\max }$ allowed such severe decrements in $F$.

At $20 \mathrm{~h}$ into the process, the probing feeding strategy was changed so that the increment in $F_{s p}$ during a pulse lasted for three minutes and after this $F$ was returned to the value before the pulse for three minutes. After this $D O_{\text {pulse }}$ was measured, $D O$ 
control started again and the decision to change $F$ taken by the controller (figure 5.5 illustrates this variation), meaning that the window for analysis of the pulse response was essentially 6 min long $\left(t_{p}+t_{o}\right)$. This modified strategy (shown in figure 8.15) gave more efficient pulses and avoiding the problem of the controller missing a $D O$ response, as the maximum of the $D O$ response now occurred during the interval in which the $D O$ response was studied rather than shortly after it.
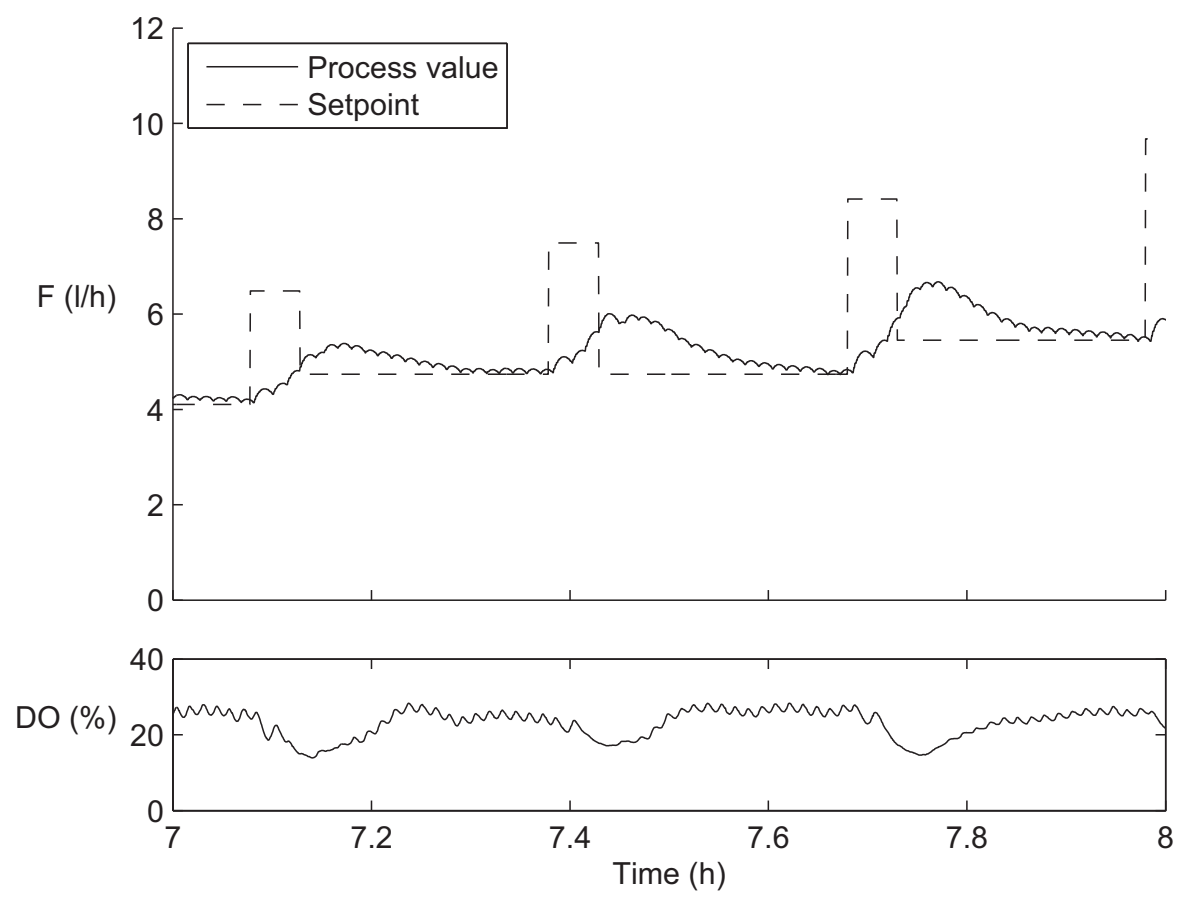

Figure 8.14 $F$ and $D O$ during pulses in the eighth development experiment.

To determine if $F$ is just below $F_{c r i t}$ as desired in a $2.5 \mathrm{~m}^{3}$ fermentation, it is possible to study $D O$ behaviour in relation to the small feed pulses occurring due to the discontinuous feed addition (as described in section 6.1). The $D O$ response to these can be evaluated similarly to the actual probing pulses caused by a change in $F_{s p}$. Based on this, the $D O$ behaviour in figure 8.15 indicates that overflow metabolism is taking place even at the baseline after an observation time has been added to the controller, while figure 8.14 indicates that no such problem is seen before this modification. In fact, the change from oscillatory behaviour to a much less noisy $D O$ curve during pulses indicates that $F$ is just below $F_{c r i t}$ when at the baseline in figure 8.14, before the addition of an observation time.

This tendency to give pseudo-responses after the addition of an observation time can explain the accumulation of glucose between 30 and $50 \mathrm{~h}$ as seen in figure 8.13. However, this appears to only have caused a very minor production of acetate during this time, indicating that it might not be a significant problem. An explanation to this is that the pseudo-response effect does not give enough of a change in $D O$ for this to count as a response to a pulse but when $F$ is just below $F_{\text {crit }}$ and up pulses therefore give a reduced $D O$ response, which would normally be slightly below $D O_{\text {reac }}$, the pseudo-response effect adds to $D O_{\text {pulse }}$ so that it becomes greater than $D O_{\text {reac }}$ and causes an increase in the $F$ baseline to a value just above $F_{c r i t}$. This would mean that the robustness of the system is not lost, as $F$ will not increase without regard to $F_{\text {crit }}$, but means that the system will converge to a value just above $F_{\text {crit }}$ rather than just 


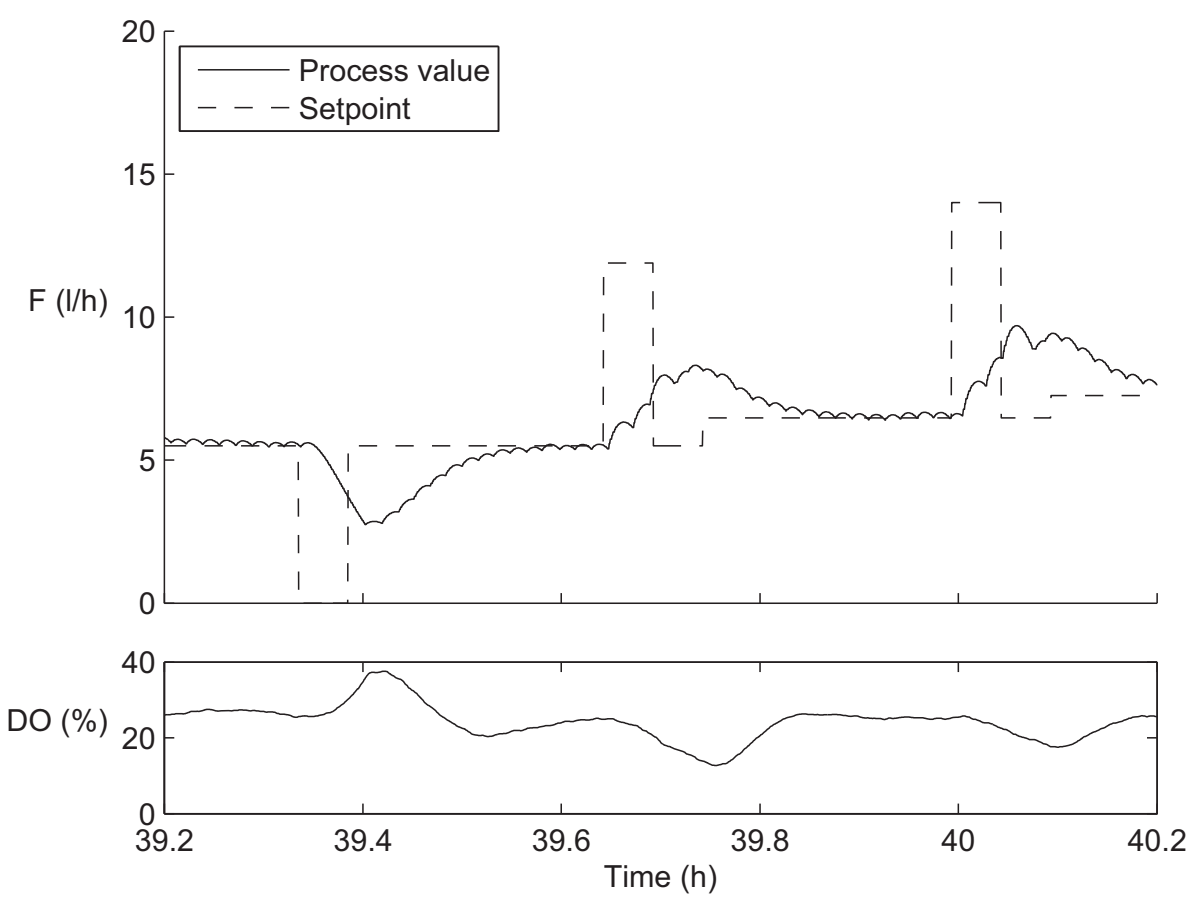

Figure 8.15 $F$ and $D O$ during pulses using the modified pulsing strategy in the eighth development experiment.

below it. The system will however be close to the point where it does not converge to a value near $F_{c r i t}$ at all and thus loses stability.

Although the probing controller did not appear to behave optimally in this experiment, with for instance the too large decrement in $F$ at $14.5 \mathrm{~h}$ and the indications of overflow metabolism after adding an observation time, the outcome of this experiment shows that it is possible to run a $B$. licheniformis fed-batch fermentation in $2.5 \mathrm{~m}^{3}$ scale using a probing controller for $F$. This outcome led to the decision to perform an evaluation experiment in $2.5 \mathrm{~m}^{3}$ scale, to get data on the outcome of a process when using the probing controller in its current incarnation.

\subsection{Discussion of development experiments}

To provide a summary of the development experiments, table 8.5 shows the different features of the controller and brief comments on how they were altered before and during the different development experiments.

\section{Dissolved oxygen control}

As shown by the tests of agitator speed and aeration rate dynamics during the second experiment (see figure 8.3), the slow agitator speed dynamics mean that it is impossible to achieve fast and good control of $D O$ using the agitator speed as the control parameter (unless these dynamics can be made faster). However, the aeration rate control in DeltaV is fast enough that a well-tuned $D O$ controller can be quite fast when using the aeration rate as the control parameter.

Another way of using the agitator speed and aeration rate in the $D O$ control, rather than using them as control parameters one at a time, would be to use mid-ranging con- 


\begin{tabular}{|c|c|c|c|c|}
\hline \multicolumn{5}{|c|}{ Feed rate control } \\
\hline Experiment & Probing control & Initial feed ramp & $D O$ safety net & $\begin{array}{l}\text { Feed pump ac- } \\
\text { tion }\end{array}$ \\
\hline 1 & Test of concept & $\mathrm{N}$ & $\mathrm{N}$ & Discontinuous \\
\hline 2 & Test of concept & $\mathrm{N}$ & $\mathrm{N}$ & Discontinuous \\
\hline 3 & $\mathrm{U}$ & $\mathrm{N}$ & $\mathrm{N}$ & $\begin{array}{l}\text { First test of con- } \\
\text { tinuous feed }\end{array}$ \\
\hline 4 & $\begin{array}{l}\text { First run from } \\
\text { start to end }\end{array}$ & $\begin{array}{l}\text { First implementa- } \\
\text { tion }\end{array}$ & $\mathrm{N}$ & Continuous \\
\hline 5 & $\mathrm{U}$ & $\mathrm{U}$ & $\begin{array}{l}\text { First implementa- } \\
\text { tion }\end{array}$ & $\begin{array}{l}\text { Continuous, } \\
\text { pump recalibrated }\end{array}$ \\
\hline 6 & $\mathrm{U}$ & $\mathrm{U}$ & $\mathrm{U}$ & $\mathrm{U}$ \\
\hline 7 & $\mathrm{U}$ & U & $\mathrm{U}$ & $\begin{array}{l}\text { Continuous, } \\
\text { pump recalibrated }\end{array}$ \\
\hline 8 & $\begin{array}{l}\text { Observation time } \\
\text { added }\end{array}$ & $\begin{array}{l}\text { Recalculated for } \\
2.5 \mathrm{~m}^{3} \text { fermenta- } \\
\text { tions }\end{array}$ & $\begin{array}{l}\text { Set to use smaller } \\
\text { decrements }\end{array}$ & Discontinuous \\
\hline
\end{tabular}

\begin{tabular}{clll}
\hline \multicolumn{4}{c}{ Dissolved oxygen control } \\
\hline Experiment & Agitator speed control & Aeration rate control & General \\
\hline 1 & $K_{c}$ changed & $K_{c}$ changed & \\
2 & $\mathrm{U}$ & $\mathrm{U}$ & $\begin{array}{l}\text { Test of deadband for } D O \\
\text { (was removed again) }\end{array}$ \\
& & $\mathrm{U}$ & \\
4 & $\mathrm{U}$ & $\mathrm{U}$ & \\
5 & $\mathrm{U}$ & $K_{c}$ and $T_{i}$ changed & \\
6 & $K_{c}$ and $T_{i}$ changed & & \\
7 & $K_{c}$ chain scheduling added & $K_{c}$ changed & Rescaled for $2.5 \mathrm{~m}^{3}$ fer- \\
8 & Gain scheduling expanded & $\mathrm{U}$ & mentor.
\end{tabular}

Table 8.5 Summary of the development experiments and the changes to the controller done before and during each experiment $(\mathrm{N}$ indicates that the feature was not used in this experiment, $\mathrm{U}$ indicates that the feature was used unmodified, as in the previous experiment).

trol. This should give the $D O$ control system the benefits of the fast aeration control dynamics at all times, but was not recognised until most experiments had been done and there was not enough time to try and implement this method, however.

Another advantage of this method would be that the aeration rate would go up at the start of the process, meaning that there should be a lower risk of having too high $\mathrm{CO}_{2}$ levels in the gas phase in the fermentor. High levels are undesirable as they can inhibit growth of the microorganisms and it is believed that this may have been an issue in the early stages of some development experiments.

To improve the $D O$ controller behaviour tuning experiments should be run, to calculate the optimal gain at different points in the process. This has not been done in the development experiments in this project, as it was considered possible to evaluate the possibilities of implementing probing control without such tuning experiments and accessing $2.5 \mathrm{~m}^{3}$ tanks to perform such experiments in proved difficult. Although tuning in 201 fermentations would have been possible this would have taken some time and the evaluation of the process would still be made for $2.5 \mathrm{~m}^{3}$ fermentations, making improved controller behaviour in 201 fermentations less relevant. 


\section{Feed rate control}

The duration of pulses was an issue which was considered many times during the development experiments. As seen in the seventh experiment, too long pulses means that $D O$ will appear to react to a pulse even though it is not actually the case, this phenomenon is referred to here as pseudo-responses. This is an effect which must be avoided for probing control to work, but in cases where the feed dynamics are slow it can be necessary to have a rather long pulse duration to achieve a sufficient increase in $F_{p v}$ for a pulse response to be registered.

Ideally, $F_{p v}$ should rapidly reach $F_{s p}$ during the pulse and just as rapidly reach the baseline after the pulse, as this means that the pulse can have a short duration. Slow dynamics when changing the feed rate as the pulse starts means that the pulse goes on for longer than would be necessary and/or that the highest/lowest (depending on pulse direction) process value of the feed rate will occur after the pulse is over. Slow dynamics when returning the feed rate to the baseline means that more (for up pulses) or less (for down pulses) feed than desired is entered into the fermenter.

The fourth development experiment was relevant as it was the first to show the controller's ability to reach and remain at a set value of $F$. In hindsight this experiment used 10 min pulses, meaning that behaviour similar to that in the seventh development experiment could be possible where $F$ is increased despite the system being at overflow until the maximum value of $F$ is reached. However, as biomass growth is slower when excess glucose is not present and $F$ did not increase to its maximum value during the fourth experiment, it seems likely that excess glucose was never present at the start of pulses and the biomass growth during an up pulse was never enough in itself to decrease $D O$ more than the value of $D O_{\text {reac }}$ during an up pulse. It may have had some effect by causing higher values of $\Delta F$ after pulses, however.

Adding an observation time after the pulse during which no $D O$ control is acting, as done in the eighth development experiment, means that measurements of $D O_{\text {pulse }}$ are done at the peak of the pulse. This means that the highest value of $D O_{\text {pulse }}$ is found and used to determine $\Delta F$, increasing the gain of the probing controller without higher amplitudes of pulses being used. This is a tradeoff against the risk of creating pseudo-responses and as shown in figure 8.15 this is an issue in the eighth experiment, meaning that shorter observation times may be desirable.

The static feed ramp at the start of the experiment is a necessary feature to decrease $D O$, but essentially undesirable since no closed-loop control of $F$ takes place during it. It would therefore be desirable to shorten it down, which could be made possible through using a higher $D O_{s p}$ at the start of the process and decreasing it stepwise when $N$ and $Q_{g a s, \text { in }}$ are sufficiently high in order to not end the use of probing control in the process earlier than necessary.

The possibility to study $D O$ oscillations caused by the discontinuous feed addition is a very useful tool for analysing the controller's behaviour, allowing many conclusions which would otherwise be hard to draw. It could presumably be used in the controller itself, this is however outside the scope of this project.

The $D O$ safety net is the mechanism which decreases $F$ to prevent oxygen limitation in the system caused by limitations in the oxygen transfer capacity of the fermentor. Using small decrements of $F$ as in the eighth development experiment, it acts as desired at almost all times; slowly increasing $D O$ until it is just above the minimum level (see figure 8.13). However, it is possible that it would be advantageous to instead use $D O$-controlled feed just as in the currently used control strategy (see chapter 7) after the limit in the fermentor's oxygen transfer capacity is reached, as overflow metabolism is then not expected to be an issue. Should it however be that a metabolic shift leading to a significantly decreased $q_{g, c r i t}$ is known to be possible, 
such a method might then lead to overflow metabolism in the process. The same is true for the safety net as it is used in the eighth development experiment, however.

\subsection{Features of the final controller}

The final version of the controller (finalised in the eighth development experiment and used in the evaluation experiment) is a time-discrete system with a $t_{\text {cycle }}$ of two seconds. The control signals are agitator speed, $N$, aeration rate, $Q_{g a s, i n}$ and feed rate, $F$. The only process parameter which effects the probing controller is the $D O$ process value, although it reads several other parameters as well for data display and storage. It is capable of controlling three parallel fermentation processes independently and could easily be expanded to control a higher number if desired.

The currently used control method is entirely DeltaV-based, using static ramps and a PI-controller for $D O$-controlled feed rate to determine setpoints for the process variables. The current strategy and the probing strategy which has been developed in this project are displayed in figures 8.16 respectively 8.17 , showing all subsystems and relevant signals to enable a comparison and visualise what has been changed and added in the control strategy during the project.

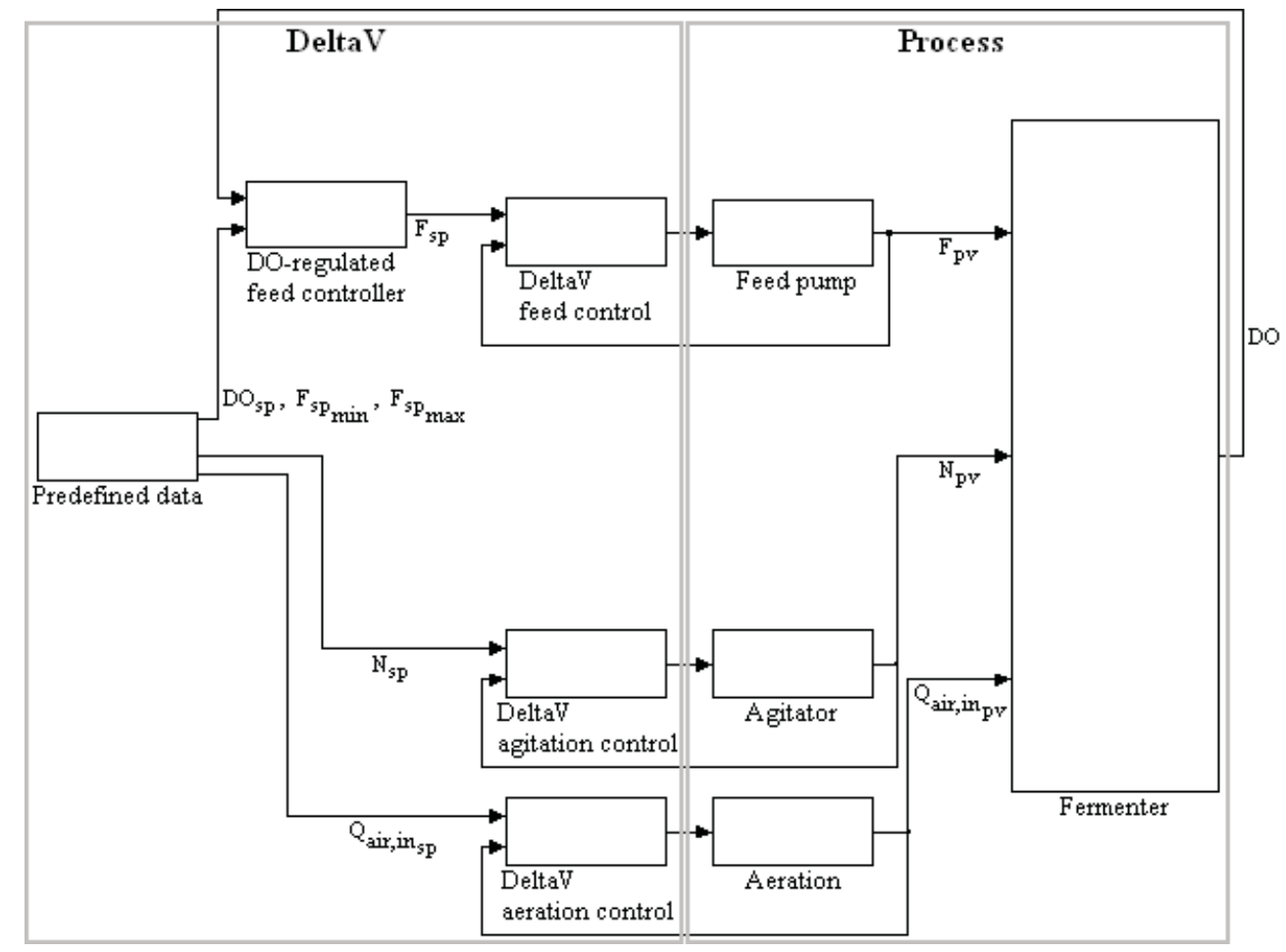

Figure 8.16 An overview of the currently used control system.

At the start of the fermentation, the controller performs an exponential (piecewise linear) feed ramp similar to the maximum ramp used during regular $B$. licheniformis fermentations (see figure 7.1), during which the aeration rate and agitator speed are kept at their respective minima. No pulses are performed as the feed ramp is static. At first the $D O$ process value is much higher than its setpoint but during the time of the ramp the biomass in the reactor is growing, leading to a decreasing $D O$. When $D O$ has reached its setpoint the static feed ramp is switched off. $D O$ is then regulated through PI control and $F$ through probing control. 


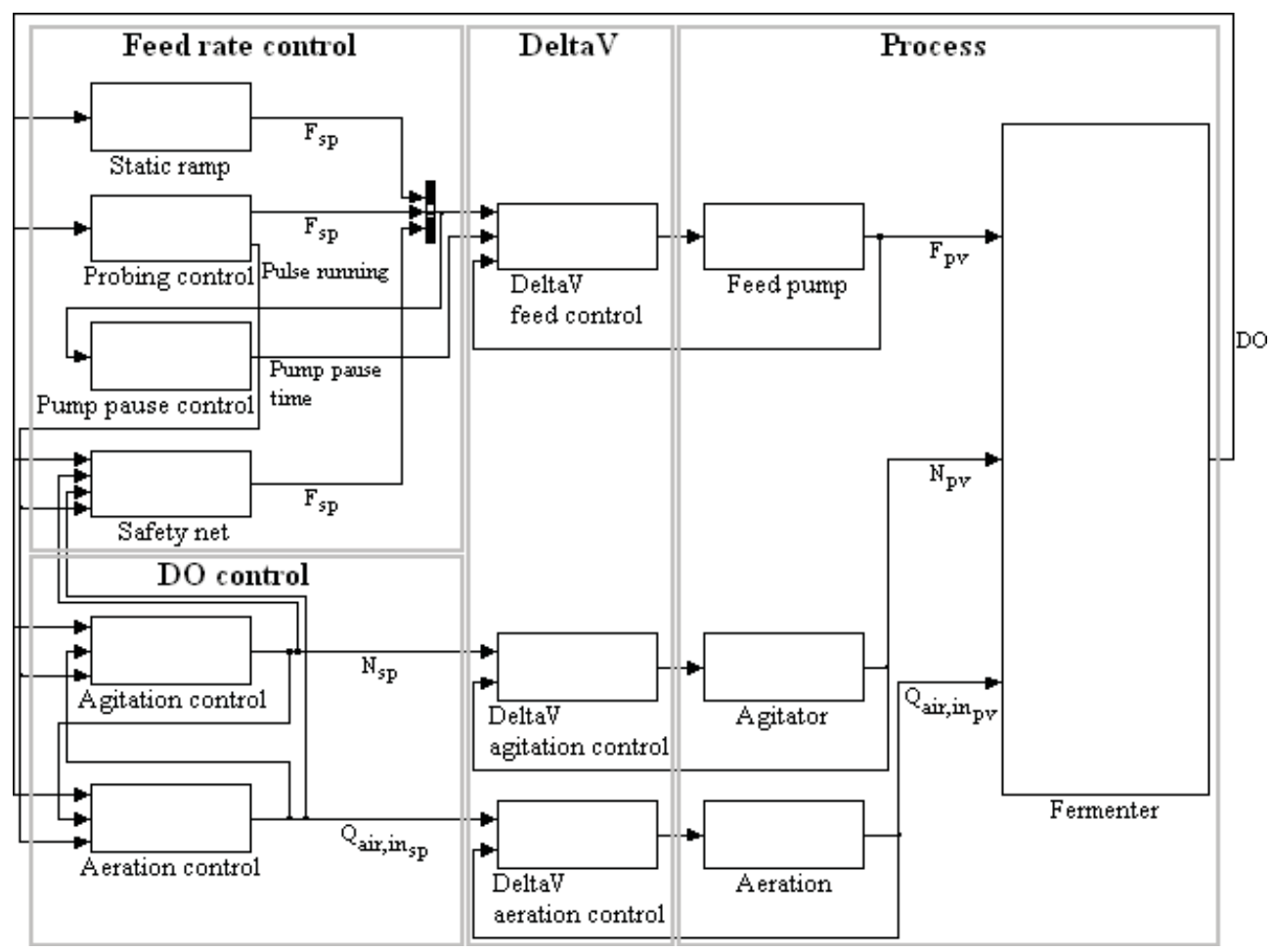

Figure 8.17 An overview of the final probing controller system.

The PI control of $D O$ uses $Q_{g a s, \text { in }}$ and $N$ as control signals, one at a time as described in the beginning of chapter 8. Gain scheduling is used for control of $N$, with $N$ as the parameter determining the gain, as described in section 8.4. Tuning of $D O$ control has not been done and it is therefore not optimal, but good enough that the probing controller can perform pulses and thereby control $F$.

To ensure $D O$ stability at the setpoint before a new pulse is started, two separate conditions must be fulfilled before the probing controller performs a pulse. To ensure proximity to the setpoint, the difference between the $D O$ setpoint and its current process value must be lower than a defined fraction of the $D O$ setpoint. To ensure stability, the difference between minimum and maximum $D O$ values in the last 200 controller cycles (equivalent to $400 \mathrm{~s}$ ) must be lower than a defined number. The $D O$ stability conditions used by the probing controller are described in equations 8.1 and 8.2 and the parameters of the final probing controller are specified in table 8.6. Equation 8.1 is similar to that used in (Åkesson 1999, Paper V), found in section 5.2.

$$
\begin{array}{r}
\frac{\left|D O-D O_{s p}\right|}{D O}<D O_{t o l} \\
\left|\min \left(D O_{i-n+1} \ldots D O_{i}\right)-\max \left(D O_{i-n+1} \ldots D O_{i}\right)\right|<\delta
\end{array}
$$

The probing controller also incorporates two features to avoid performing unnecessarily long pulses. During an up pulse, if the current $D O$ is sufficiently low that $\Delta F$ after the pulse will reach its maximum, it is not necessary to let the pulse continue for longer and it is therefore stopped immediately. Similarly, in the case of a down pulse, if the current $D O$ gives a $D O_{\text {pulse }}$ greater than $D O_{\text {reac }}$ the pulse is stopped immediately as it will then count as giving a $D O$ response irrespective of what other $D O$ values might be measured during the pulse. The former of these features does not only have the purpose of preventing unnecessarily long pulses; it also ensures that 


\begin{tabular}{ll}
\hline Parameter & Value \\
\hline$t_{c y c l e}$ & $2 \mathrm{~s}$ \\
\hline$D O$ control & \\
\hline Agitation control & \\
$K_{c}$ & $100-140 \mathrm{rpm}: 0.167$ \\
& $140-185 \mathrm{rpm}: 0.333$ \\
& $185-215 \mathrm{rpm}: 0.667$ \\
& $215-275 \mathrm{rpm}: 1$ \\
$T_{i}$ & $100-275 \mathrm{rpm}: 0.4$ \\
\hline Aeration control & \\
$K_{c}$ & 5 \\
$T_{i}$ & 0.4 \\
\hline$F$ control & \\
\hline Pump pause time & $0-10 \mathrm{l} / \mathrm{h}: 30 \mathrm{~s}$ \\
& $10-19 \mathrm{l} / \mathrm{h}: 45 \mathrm{~s}$ \\
\hline Feed ramp & \\
interpolation points & \\
$0 \mathrm{~h}$ & $0.6 \mathrm{l} / \mathrm{h}$ \\
$3 \mathrm{~h}$ & $2.8 \mathrm{l} / \mathrm{h}$ \\
$5 \mathrm{~h}$ & $5 \mathrm{l} / \mathrm{h}$ \\
\hline Probing controller & \\
parameters & \\
$D O_{s p}$ & $25 \%$ \\
$t_{p}$ & $3 \mathrm{~min}$ \\
$t_{O}$ & $3 \mathrm{~min}$ \\
$t_{c, \text { min }}$ & $15 \mathrm{~min}$ \\
$D O_{\text {reac }}$ & $4 \%$ \\
$\gamma_{p}$ & 0.775 \\
\hline & 1 \\
\hline & \\
& \\
& \\
&
\end{tabular}

Table 8.6 Parameters for the final controller.

$D O$ does not reach zero during a pulse, which is considered unnecessary although not a major problem as the ensuing $D O$ limitation would only last for a very short time.

It should be noted that it was deemed necessary to increase $t_{c, \min }$ from 5 min to 15 min after the eighth experiment, as this was how long it took for $F_{p v}$ to return to near $F_{s p}$ after a pulse. Another way to solve this problem would be to make stability conditions for $F$ similar to those for $D O$, however, the ideal solution would be to increase the speed of the dynamics for $F$ if that would be possible. Also, the feed pause time for values of $F$ over $10 \mathrm{l} / \mathrm{h}$ was decreased from $60 \mathrm{~s}$ to $45 \mathrm{~s}$ after the eighth development experiment in order to give smaller oscillations in $F_{p v}$, which was deemed necessary. 


\section{Evaluation experiment}

The purpose of the evaluation experiment was to run and study a pilot scale process regulated from start to end by the final variant of the probing controller without significant manual input during the process, in order to allow relevant comparisons to the existing process. The modified probing strategy developed during the eighth development experiment was implemented from the start in this experiment, which was also set up similarly to said development experiment.

The static ramp at the start of the fermentation had its slope increased at several points in time as the behaviour of the $D O$ signal indicated that $F$ was below $F_{c r i t}$ (as described in section 8.6). Beyond this and some minor changes to the $D O$ safety net, no manual modifications were done during the experiment.

The outcome of this experiment was very much like what was expected from a probing controller with a $D O$ safety net; $F$ was increased in an exponential manner until it reached its maximum, after which the safety net soon started acting to decrease $F$ to a level where $D O$ remained stable. An overview of the fermentation can be seen in figure 9.1.

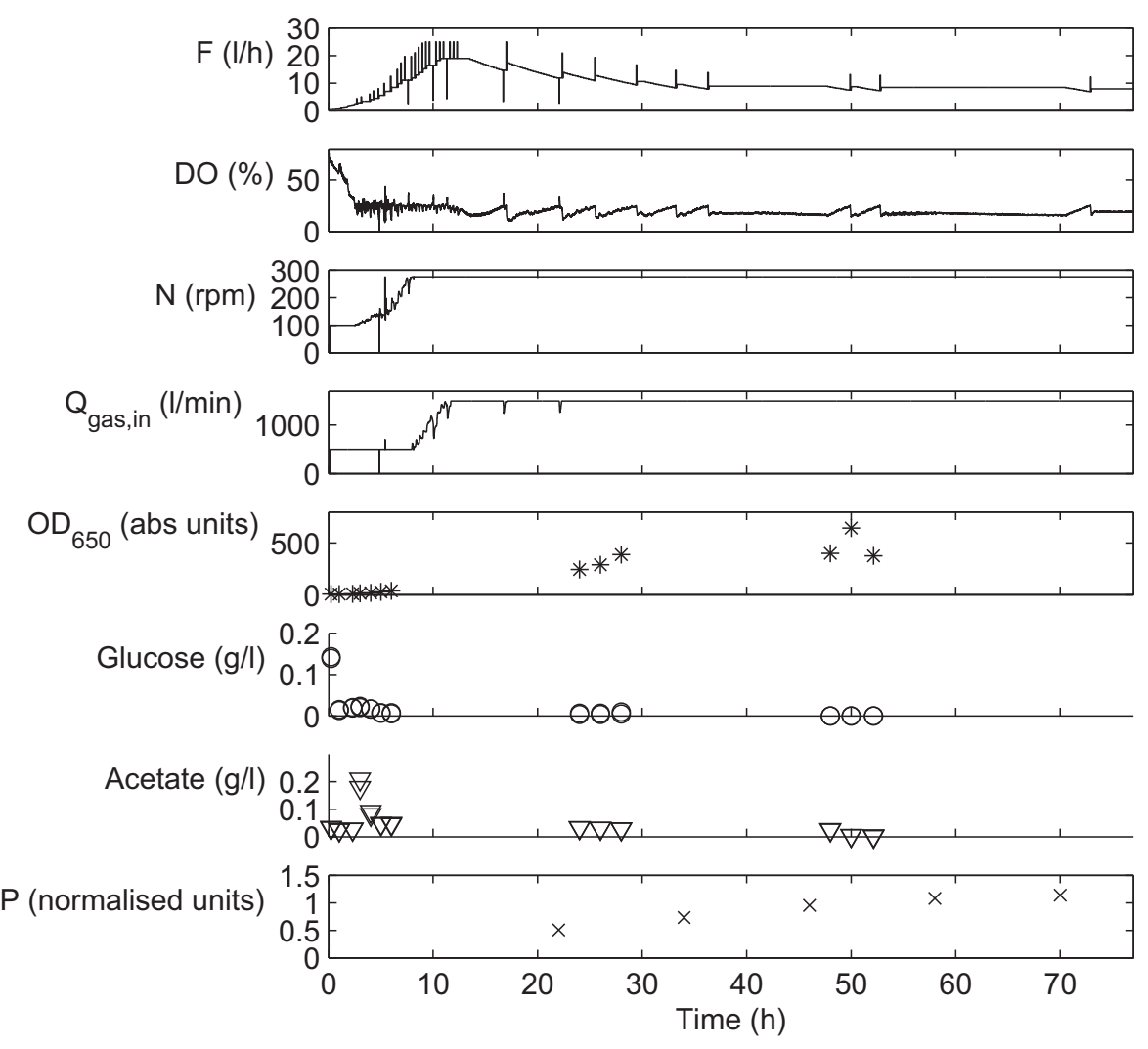

Figure 9.1 Overview of the evaluation experiment.

In this experiment the safety net was modified to decrease $F$ until $D O$ reached its setpoint at $25 \%$ so that new pulses could be performed, rather than decreasing $F$ until $D O$ was above the minimum allowed $D O$ of $10 \%$ as was done in the eighth development experiment. This gives a worse behaviour from a process efficiency 
point of view, as $F$ goes up and down more than would be necessary, but it was done in order to continuously evaluate whether overflow metabolism occurred in the system (this would only be possible if a metabolic shift occurred in the bacteria or if the biomass concentration had decreased significantly). As no overflow metabolism could be detected at any point in time, it seems safe to say that the type of safety net used in the eighth development experiment would be better to use.

\subsection{Discussion of evaluation experiment}

\section{Process robustness}

Process robustness in this case is viewed as the ability of the controller to avoid crashes in the fermentation, despite varying process conditions. To determine for sure that the process is robust when using probing control, enough fermentations must be run using it to allow a comparison with the crash rate of the existing process control system. Ideally, different starting conditions such as a lower than normal initial value of X should also be tested to see if it is possible for the controller to compensate for such variations. This has not been possible to do within the time frame of this project due to the large number of fermentations required, therefore no definitive conclusions can be made regarding the robustness of the process, although the outcome of the experiments gives an indication. Developing an accurate model of the process and studying the robustness characteristics of the closed-loop system would be another way of doing this, but would likely require a large number of fermentations to achieve a useful, well-fitted model and such a model might still not incorporate all factors which influence the outcome of the process.

A comparison between the evaluation experiment and the reference experiment, as seen in figure 9.2, shows that in both experiments only very low amounts of acetate are accumulated during the first hours of the fermentation and the acetate is then rapidly consumed. It is known that the existing control method does sometimes lead to crashes, presumably due to production of overflow metabolites such as acetate, so it is clear that the experiments do not give the whole picture. The question here is whether the probing control strategy can be expected to improve process robustness based on the experimental results and what is known about the process and the different controllers.

As mentioned in the discussion of the development experiments, the length of $t_{p}$ and $t_{o}$ is a tradeoff between pulse efficiency and the risk of seeing pseudo-responses which would lead to acetate accumulation (as seen in the seventh development experiment). No acetate accumulation over time has been seen after the static ramp in the evaluation experiment, showing that although $t_{p}$ and $t_{o}$ are not necessarily optimal and it is possible that $D O_{\text {pulse }}$ values have been somewhat increased due to pseudoresponse effects, their current values do not appear to have led to long-term acetate accumulation due to the occurrence of pseudo-responses. It would be beneficial to also have such samples taken around the point in time when $F$ is as highest, however this has not been a possibility in practice due to work times at the fermentation pilot plant. To see if acetate is accumulated in short-term and then consumed, samples should be taken at considerably shorter intervals than has been done in this project.

Figure 9.2 shows that acetate is present shortly after the end of the static feed ramp at $2.5 \mathrm{~h}$ in the evaluation experiment and that it is consumed during the following two hours, when the probing control acts. At the same time, $F$ is increased exponentially. This does not in itself prove that the probing controller successfully places $F$ just 


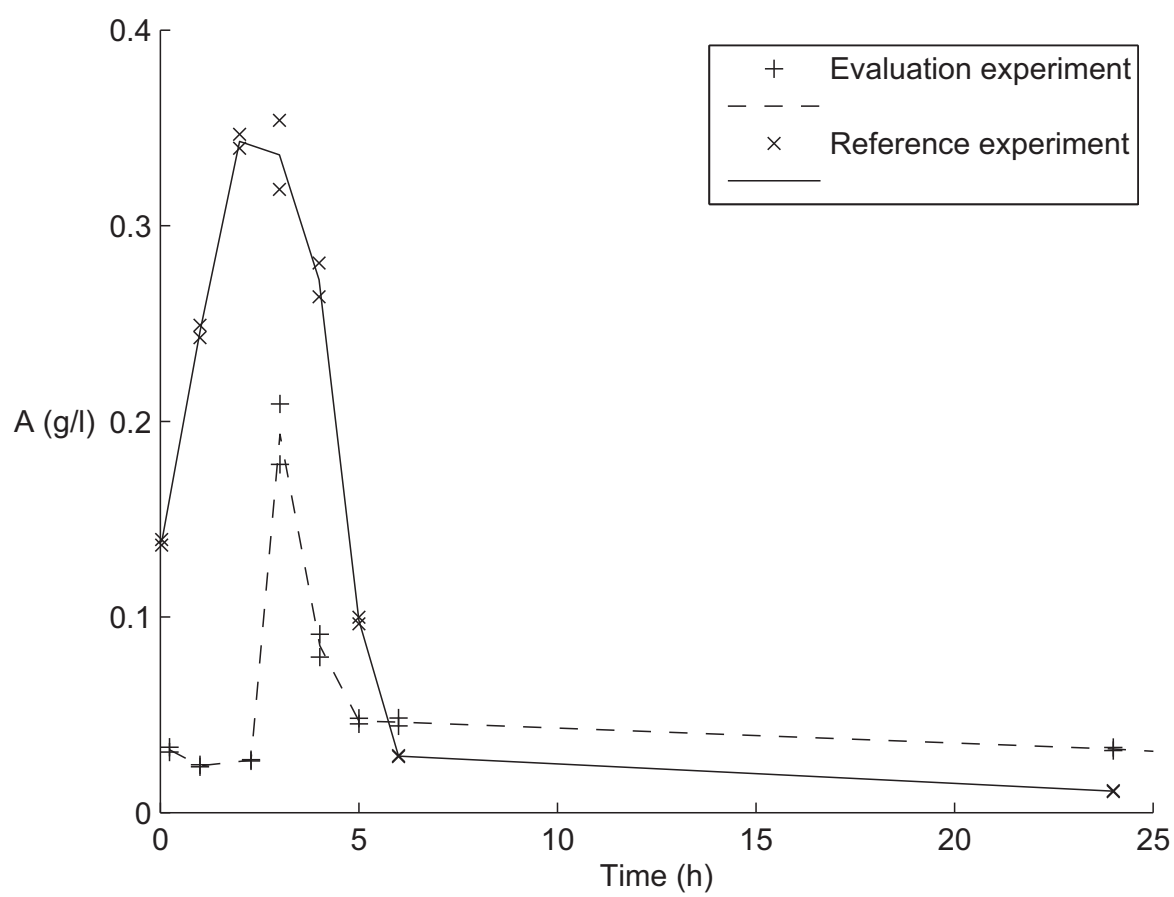

Figure 9.2 Acetate levels in the first $25 \mathrm{~h}$ of the evaluation and reference experiments.

below $F_{\text {crit }}$; the same effect would be seen if the gain of the probing controller is too low to keep up with the exponential growth of the bacteria and $F$ is thus far below $F_{\text {crit }}$.

However, as seen in figure 9.3, while at the baseline the discontinuous feed addition gives cause to $D O$ oscillations with the same period as that of the feed pulses, but during an up pulse these oscillations cannot be seen. Following the theory for probing control (similar to as in the eighth development experiment), a lack of $D O$ response indicates that $F_{c r i t}$ has been exceeded, thus meaning that the baseline is just below $F_{\text {crit }}$. It should be noted that these $D O$ oscillations are influenced by the speed of the $D O$ control; the oscillations appear to be more pronounced when using agitator speed as the control parameter rather than using aeration rate, as $D O$ control using aeration rate as the control parameter is fast enough to counteract the oscillations somewhat.

Based on these observations, it appears that the probing controller acts as desired, detecting $F_{c r i t}$ and setting $F$ to a value close to but below it. This should be a guarantee for stability in the process, as long as the $D O$ controller can perform well enough that pulses can be performed.

\section{Comparison of process efficiency}

Even if the probing controller successfully sets $F$ to a value slightly below $F_{\text {crit }}$ after each pulse, this does not necessarily mean that the process is run optimally. The $F$ baseline is linear, only varied just after a pulse, and while $F$ remains constant between pulses the actual feed demand can change during this time. Depending on the rate of biomass growth and the length of $t_{c}$, the feed demand can change more or less in the time between two pulses. In addition, the pulses themselves can cause small disturbances in the process, although these can be minimised using as small pulses as possible. These are inherent disadvantages of probing control, but if the strategy can keep $F$ close to as high as possible while avoiding overflow metabolism the efficiency 

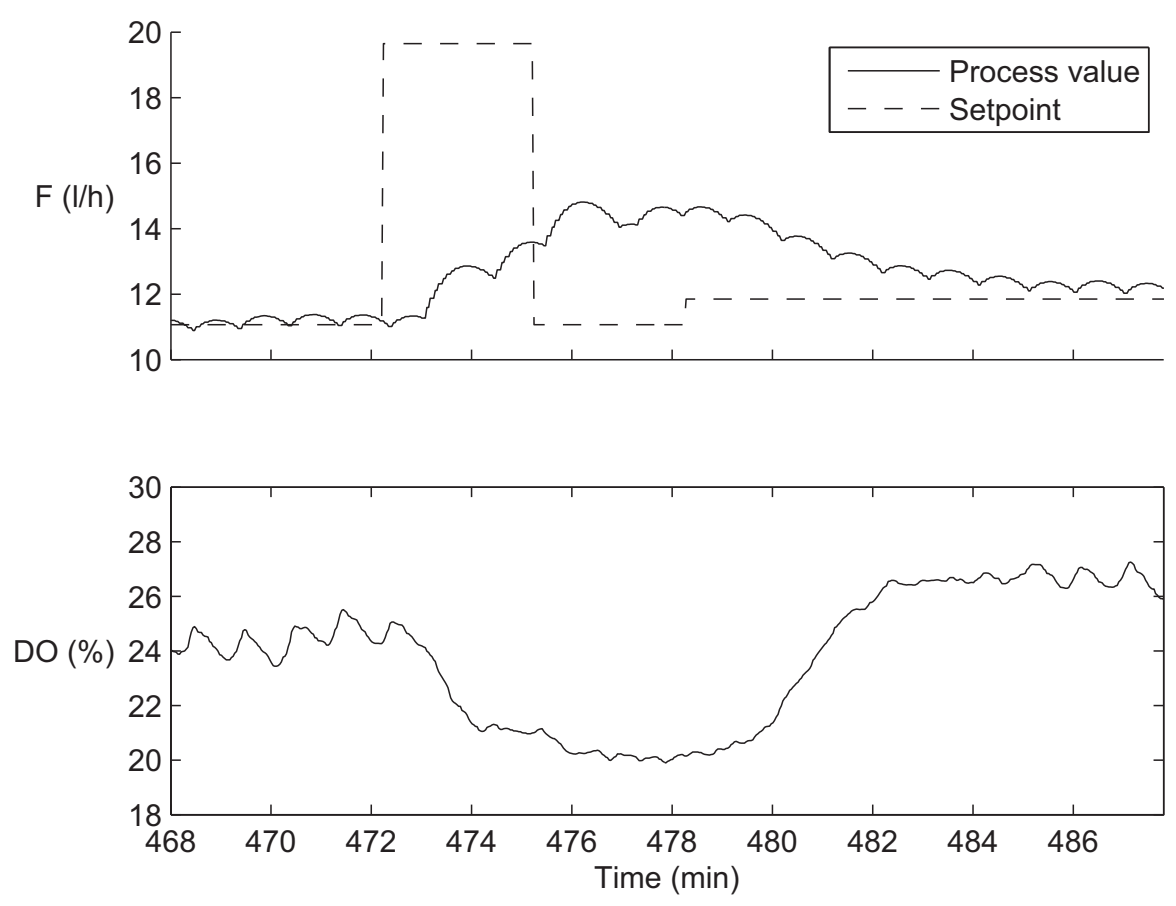

Figure 9.3 DO behaviour before, during and after a pulse in the evaluation experiment.

of the process can of course improve compared to other strategies, even in a process using a complex medium as shown in (Velut 2005).

The most direct measurement of process efficiency is the product activity. However, this parameter is only measured at long intervals and not at the same time as other off-line measurements are done, meaning that it can be difficult to relate to other results. The protein activity data (normalised by the highest measured concentration in the reference experiment) indicates that the process controlled by probing control gives significantly higher product concentrations and should therefore be an improvement over the existing control strategy, as seen in figure 9.4. However, the reference experiment was performed in a 201 fermentor rather than a $2.5 \mathrm{~m}^{3}$ fermentor as the evaluation experiment and scaling issues are a possibility here, meaning that differences in process yield may in part be due to the scaling. Also, it is possible that the problems at $14.5 \mathrm{~h}$ into the reference experiment (see section 7.1) would have led to a decreased product yield. Any such effects are not expected to be major however and it is therefore assumed that the probing controller can achieve product yields which are at least similar to those given when using the existing control strategy.

Another means of comparing the evaluation and reference experiments is to look at cell mass concentrations. $O D_{650}$ is known to have a fairly linear relation to the cell mass concentration (see section 8.3), so this parameter can be studied to make a relevant comparison.

Although fast growth of the biomass is desirable, it does not give the whole picture. If the system is at overflow, the biomass can grow at $\mu_{\max }$ for a while but in the long run the microbial growth will be inhibited by the overflow metabolism products. Therefore a comparison of biomass concentrations can only be considered relevant if the system is not at overflow. As seen in figure 9.2 acetate levels are low in both cases, indicating that overflow metabolism should not be a concern.

Figure 9.5 compares $O D_{650}$ in the evaluation and reference experiments and shows 


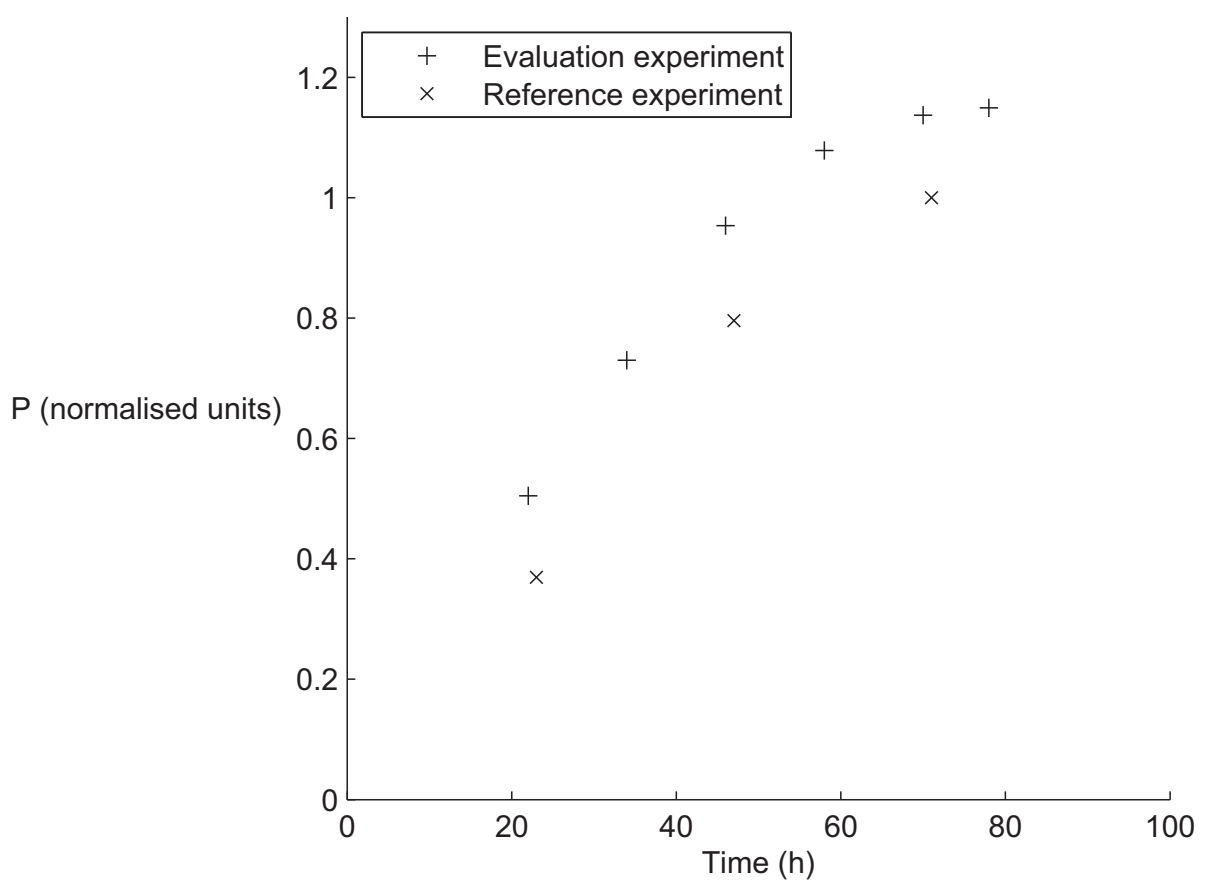

Figure 9.4 Comparison of product activity levels in evaluation and reference experiments.

that $O D_{650}$ in the evaluation experiment is about 1.13 times that in the evaluation experiment after $6 \mathrm{~h}$. Any scaling issues may of course influence biomass, not just product yield, so this difference may also be explained by the difference in scale between the reference experiment and the evaluation experiment. Scaling issues are not believed to be major however, meaning that the yields when using the different strategies in the same process should be fairly close to each other.

Thus it appears that the objectives for the probing controller have been reached. It is possible to implement it in the process, it seems to give a robust process and it can give fairly good process yields even without being particularly optimised. How far it is possible to optimise it and if it can be a viable replacement for the current control strategy cannot be said at this stage however, but would require more work focused on optimisation and evaluation of process stability and yield. 
Chapter 9. Evaluation experiment

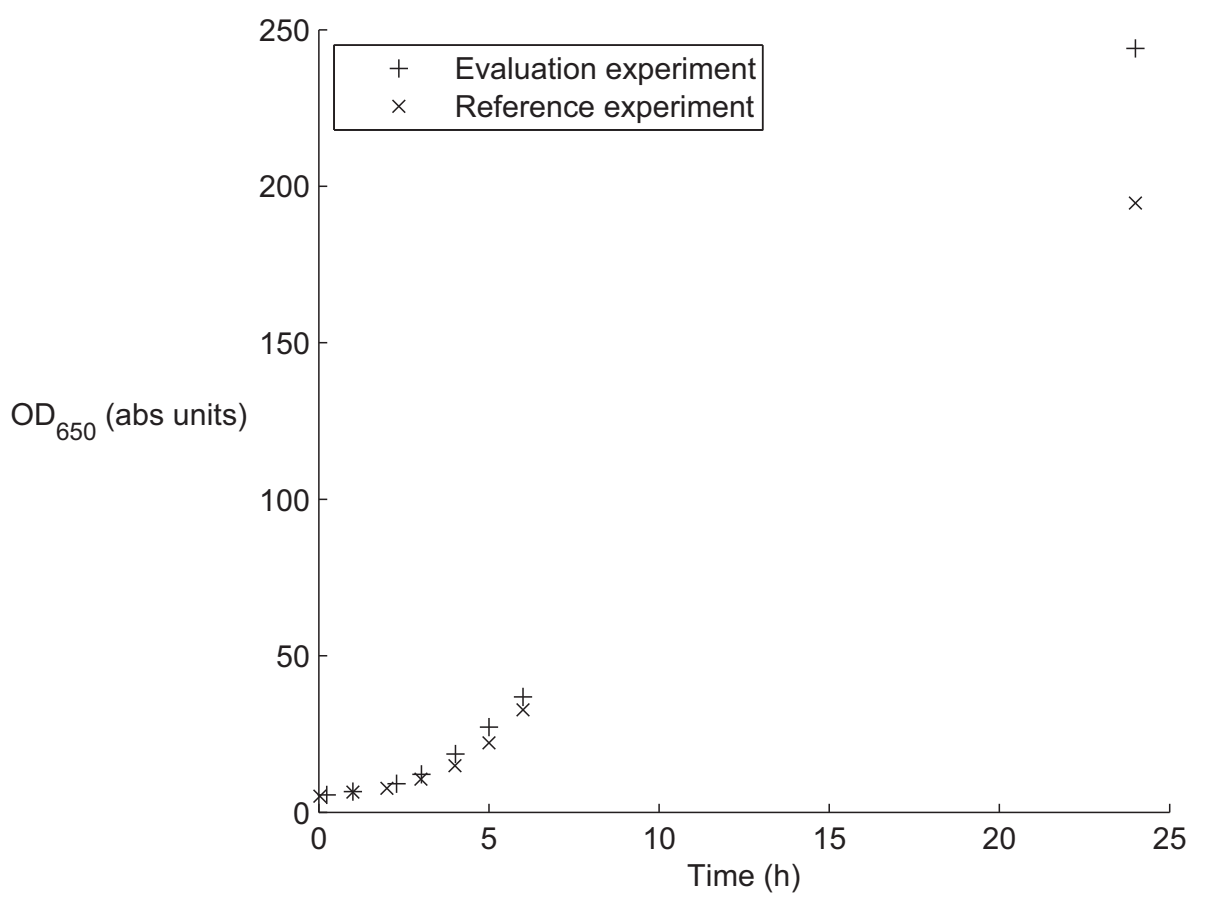

Figure 9.5 Comparison of $O D_{650}$ in evaluation and reference experiments. 


\section{Future work}

\subsection{Proof of improved process robustness}

Although the outcome of the experiments in this project, combined with knowledge of the process, indicates that probing control will make the process more stable this is still to be proven experimentally. As overflow metabolism can be an issue during the outgrowth phase before the oxygen transfer capacity of the system is reached, this period should be studied closer. With the currently used control strategy of $D O$ controlled $F$, overflow metabolism can be expected if $\mu$ during the first hours of the outgrowth phase and/or the initial X should for one reason or another be lower than normal. It would therefore be appropriate to perform experiments under one or both of these conditions and compare the outcome when using $D O$-controlled $F$ and when using probing control. It is also possible to make a direct measurement of the robustness of the process by running fermentations using these different control strategies and comparing the process crash rates.

Both of these methods have a problem in that they would possibly require a large number of fermentations in order to see any statistically significant differences. Creating a model of the system and simulating the use of the two control strategies would be one way of simplifying this process, although as mentioned before fitting a model to the process under all relevant conditions is expected to be difficult.

\subsection{Improving control of the process}

A few possible ways of improving the performance of the probing controller developed in this project have been discussed in section 8.6 and are summarised in table 10.1 .

\begin{tabular}{ll}
\hline Possible means of improvement & Expected improvements \\
\hline Increasing speed of $F$ dynamics & Necessary to perform pulses more often \\
Tuning of $D O$ control & Pulses performed more often \\
Speed up DeltaV control loops (if possible) & Pulses performed more often \\
Using mid-ranging control for $D O$ & Pulses performed more often, lower risk of $\mathrm{CO}_{2}$ \\
& accumulation in gas phase \\
Finding optimal values of $t_{p}$ and $t_{O}$ & More robust controller \\
Varying $D O_{s p}$ during the fermentation & Time when static feed ramp and/or safety net is act- \\
& ing can be decreased \\
$D O$-controlled feed rather than $D O$ safety net & Better control of $F$ after the oxygen transfer capac- \\
& ity of the fermentor is reached \\
\hline
\end{tabular}

Table 10.1 A summary of direct means of improving the probing controller developed in this project. 


\subsection{Improving the probing control strategy}

As mentioned in sections 8.5 and 9.1, studying $D O$ behaviour in relation to the inherent feed pulses caused by the discontinuous feed addition appears to be a way of determining whether or not the system is at overflow. It is then natural to look at how this could be used as an alternative probing control strategy, as it would have several benefits over the current probing control strategy.

Although the current probing control strategy can successfully detect the saturation in oxidative capacity and thus optimise $F$ and avoid overflow metabolism, it has an inherent weakness in that it takes time for the system to adjust to the changes taking place at the start and end of pulses. A probing control strategy where $F_{s p}$ is not changed due to pulses would for instance have the advantage that changes in the baseline can be performed more often, as it is not necessary to wait for the system to return $F$ and $D O$ to their respective setpoints after a pulse has been performed. Figure 10.1 shows $F$ and $D O$ behaviour in the eighth experiment, displaying clearly that the $D O$ oscillations have the same frequency as the feed pulses.
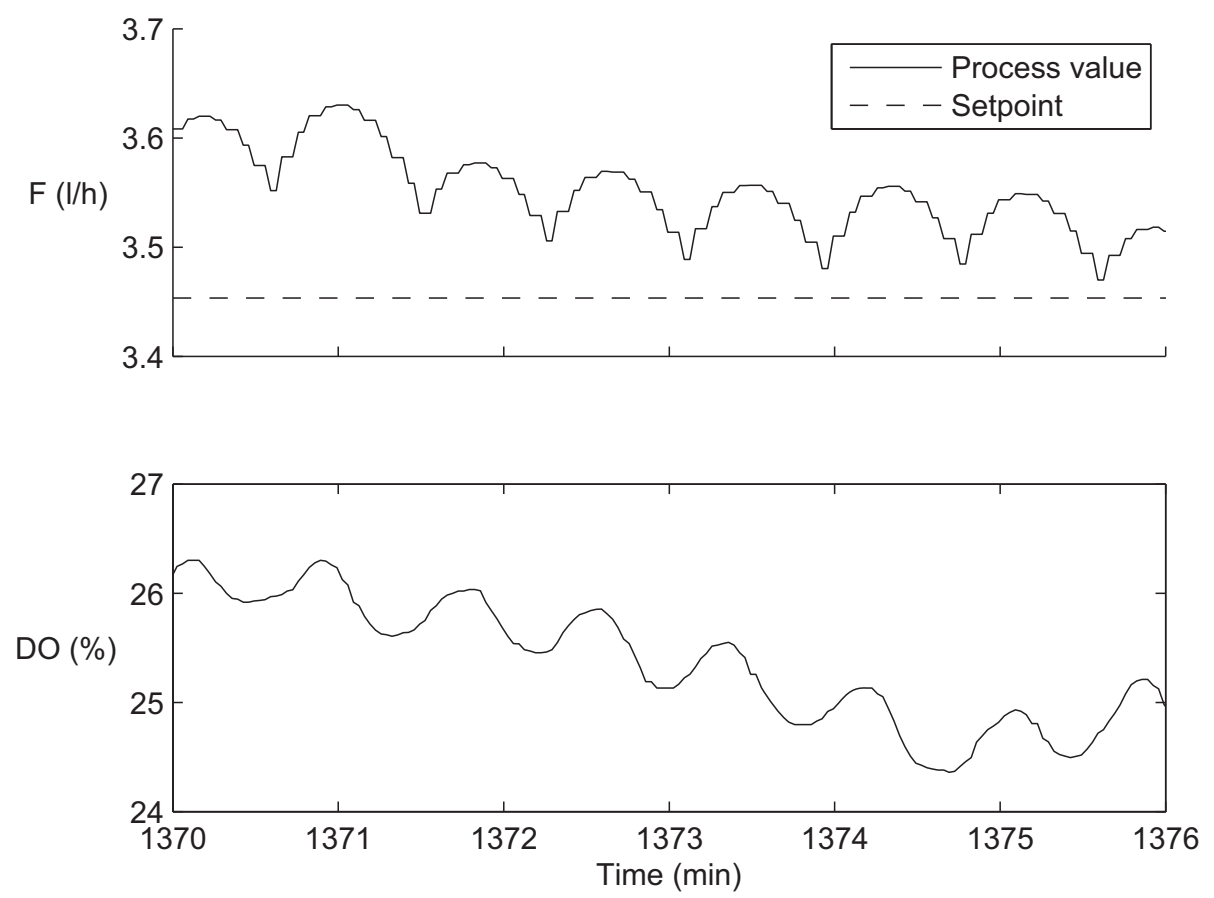

Figure 10.1 DO oscillations caused by the non-continuous feed in the eighth development experiment.

To successfully create a controller based on this strategy, two things must be achieved. Firstly, the amplitude of the oscillations or at least the presence of them must be possible to measure despite disturbances and noise. Secondly, a function for calculating how far from $F_{\text {crit }}$ the current value of $F$ is (and thus deciding how to control the feed rate) from the amplitude of the oscillations must be found.

The frequency of the oscillations is well known, as it is the same as the frequency of the feed rate pulses (although there is a phase delay), meaning that the amplitude can be found for instance by creating a Fourier transform of the $D O$ curve and studying the value given for the current frequency of the feed rate pulses. Finding a function for changing $F$ based on the amplitude can presumably be done through 
modelling and experiments.

In addition, the duration of amplitude measurements needs to be considered. In the large-scale fermentations, the period of the feed pulses is minimum $30 \mathrm{~s}$. As described in section 8 this period must be increased to achieve high values of $F$, however. What determines the necessary duration of the amplitude measurements is the length of this period as well as how many periods are necessary to calculate the current amplitude of the oscillations with sufficient precision.

The question of $D O$ control during amplitude measurements should also be considered. As discussed in section 9.1, fast $D O$ control will counteract the pulses, meaning that $D O$ control must be switched off during amplitude measurements. Slow $D O$ control means that oscillations can be observed without switching it off, however even slow $D O$ control will still interfere somewhat with the oscillations.

What makes it possible to use this type of probing control strategy is the existence of modern $D O$ electrodes with a low time constant. When probing control of fedbatch cultivations was first looked at, existing $D O$ electrodes were not considered fast enough to be suitable for this (Hagander 2010).

It should be stressed that in automatic control systems like the $D O$-controlled feed system used at Novozymes, the $D O$ signal is heavily filtered. To observe the $D O$ oscillations caused by discontinuous feed addition such filtering should not be used and data sampling should be performed at short intervals, as done in most development experiments as well as in the evaluation and reference experiments. 


\section{Conclusions}

The outcome of this project shows that it is possible to successfully perform a pilot scale $\left(2.5 \mathrm{~m}^{3}\right)$ B. licheniformis fed-batch fermentation controlled by probing control. Even though the controller has not been optimised in this implementation, it has shown itself capable of achieving yields similar to those when using the currently used control strategy. There is clearly room for optimisation of the controller through several different means, such as improving dissolved oxygen control and testing different variations of the probing controller, although it is hard to predict how much this can improve process robustness and yield.

Definitive statements regarding the robustness of the process when controlled through probing control cannot be made, as a large number of fermentations would be required to determine whether the crash frequency would decrease compared to the existing control strategy. However, the probing controller has shown itself capable of directly detecting feed rates both above and too far below the critical feed rate for overflow metabolism in pilot scale $B$. licheniformis fermentations and adjust the feed rate accordingly. It is therefore reasonable to expect that the concept can be used to avoid crashes caused by accumulation of acetate due to overflow metabolism in the process while still achieving a good yield.

Based on the observations made during the project, it also appears possible to improve the probing control strategy itself by not using pulses caused by changes in the feed rate setpoint but rather utilising the pulses caused by the discontinuous feed addition to the fermentor. These pulses appear to give cause to oscillations in the dissolved oxygen level when the feed rate is below the critical limit for overflow, with an amplitude apparently related to the distance between the current feed rate and the critical level, which could be used to control the feed rate. 


\section{References}

Åkesson M. (1999): Probing Control of Glucose Feeding in Escherichia coli Cultivations. Doctoral thesis. Lund University. Department of Automatic Control.

Åkesson M., P. Hagander (2000): A simplified probing controller for glucose feeding in Escherichia coli cultivations. In: Proceedings of the $39^{\text {th }}$ IEEE Conference on Decision and Control. Sydney, Australia. pp. 4520-4525.

Dauner M., T. Storni, U. Sauer (2001): Bacillus subtilis Metabolism and Energetics in Carbon-Limited and Excess-Carbon Chemostat Culture. Journal of Bacteriology, 183:7308-7317.

de Maré L. (2006): Feeding Strategies Based on Probing Control for E. coli and V. cholerae Cultivations. Doctoral thesis. Lund University. Department of Automatic Control.

Hagander P. (2010): Personal communication.

Henes B., B. Sonnleitner (2007): Controlled fed-batch by tracking maximum culture capacity. Journal of Biotechnology, 132:118-126.

Lidén G. (2002): Understanding the bioreactor. Bioprocess and Biosystems Engineering, 24:273-279.

Luli G.W., W.R. Strohl (1990): Comparison of Growth, Acetate Production, and Acetate Inhibition of Escherichia coli Strains in Batch and Fed-Batch Fermentations. Applied and Environmental Microbiology, 56:1004-1011.

Neyra C., L.A. Atkinson, O. Olubayi, L. Sadasivan, D. Zaurov, E. Zappi (1996): Novel microbial technologies for the enhancement of plant growth and biocontrol of fungal diseases in crops. Cahiers Opt. Méd., 31:447-456.

Nielsen J., J. Villadsen, G. Lidén (2003): Bioreaction Engineering Principles 2nd ed. New York, NY, USA: Kluwer Academic / Plenum Publishers.

Rey M.W., P. Ramaiya, B.A. Nelson, S.D. Brody-Karpin, E.J. Zaretsky, M. Tang, A. Lopez de Leon, H. Xiang, V. Gusti, I. Groth Clausen, P.B. Olsen, M.D. Rasmussen, J.T. Andersen, P.L. Jørgensen, T.S. Larsen, A. Sorokin, A. Bolotin, A. Lapidus, N. Galleron, S.D. Ehrlich, R.M. Berka (2004): Complete genome sequence of the industrial bacterium Bacillus licheniformis and comparisons with closely related Bacillus species. Genome Biol., 5:R77.

Schallmey M., A. Singh, O.P. Ward (2004): Developments in the use of Bacillus species for industrial production. Can. J. Microbiol., 50:1-17.

Veith B., C. Herzberg, S. Steckel, J. Feesche, K.H. Maurer, P. Ehrenreich, S. Bæumer, A. Henne, H. Liesegang, R. Merkl, A. Ehrenreich, G. Gottschalk (2004): The complete genome sequence of Bacillus licheniformis DSM13, an organism with great industrial potential. J Mol Microbiol Biotechnol, 7:204-211.

Velut S. (2005): Probing control. Analysis and Design with Application to Fed-Batch Bioreactors. Doctoral thesis. Lund University. Department of Automatic Control. 
Voight B., T. Schweder, D. Becher, A. Ehrenreich, G. Gottschalk, J. Feesche, K.H. Maurer, M. Hecker (2004): A proteomic view of cell physiology of Bacillus licheniformis. Proteomics, 4:1465-1490.

Wikipedia, the free encyclopedia (2010): Novozymes.

Found at: http://en.wikipedia.org/wiki/Novozymes, visited 2010-03-29.

Wittenmark B. (1995), Adaptive dual control methods: an overview. In: IFAC Symp. on Adaptive Syst. in Control and Signal Proc. pp. 67-72.

Xu B., M. Jahic, S.O. Enfors (1999): Modeling of Overflow Metabolism in Batch and Fed-Batch Cultures of Escherichia coli. Biotechnol. Prog., 15:81-90. 


\section{A. Fermentation summary}

This appendix contains information on which fermentations were run in which fermentor during which experiment, as summed up in table A.1.

\begin{tabular}{lllllll}
\hline Experiment & & & \multicolumn{5}{c}{ Fermentors } \\
\hline & & $\mathbf{1 0 7 M}$ & $\mathbf{1 0 8 M}$ & $\mathbf{1 0 9 M}$ & $\mathbf{1 1 0 M}$ & 32C \\
\hline Development experiments & 1 & & PBFM70 & PBFM71 & PBFM72 & \\
& 2 & PBFM73 & PBFM74 & PBFM75 & & \\
& 3 & & PBFM76 & PBFM77 & \\
& 4 & PBFM78 & PBFM79 & PBFM80 & \\
& 5 & PBFM81 & PBFM82 & PBFM83 & Feed rate study \\
& 6 & & & PBFM84 & \\
& 7 & & & PBFM85 & PBF1031 \\
Evaluation experiment & 8 & & & & & PBF1032 \\
Reference experiment & & & & & & \\
\hline
\end{tabular}

Table A.1 A summary of all fermentations run during the experimental part of the project. 


\section{B. Sampling procedures}

The sampling procedures during the experiments in the project were kept as consistent as possible. No major deviations from what is described below occurred.

\section{General}

1. Steam which keeps the outlet sterile is switched off $10-15$ min before taking out the sample, to allow it to cool down so that the sample is not harmed.

2. The sample is poured from the fermentor into a $100 \mathrm{ml}$ plastic flask. The amount taken is about $50-75 \mathrm{ml}$, but varies based on which measurements will be performed on that sample.

3. All handling of sample liquid in non-sealed containers is performed in a laminar air flow hood.

Dry weight measurements

1. For these measurements, triple samples are taken from each tank. For each dry weight sample, a pre-weighed tube is taken from an exsicator and placed on a scales with a precision of $10^{-4} \mathrm{~g}$. Using a Pasteur pipette, it is filled with sample liquid from the plastic flask until the total weight is approximately $10 \mathrm{~g}$ greater than the weight of the empty tube. The final weight is noted down and the tubes sealed with plastic lids.

2. The tubes are centrifuged at $2800 \mathrm{rpm}$ and $5{ }^{\circ} \mathrm{C}$, for 20 minutes. The supernatant is poured off and destilled water is added to give a final volume approximately the same as before removing the supernatant. The liquid is mixed by a plastic loop, in order to avoid any part of the pellet sticking to the loop the last of the distilled water is used to clean the loop while holding it over the tube. Then the tubes are sealed and centrifuged again, as per the first centrifugation step.

3. After the second centrifugation, the supernatant is poured off and the samples are dried at $130^{\circ} \mathrm{C}$ for at least 16 hours.

4. The dried samples are weighed on the same scales as before drying.

5. The level of biomass in the tank is calculated through equation B.1.

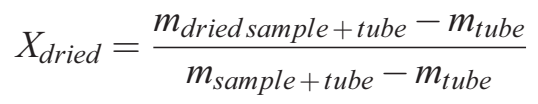

Measurement of optical density

1. The spectrophotometer was calibrated at $650 \mathrm{~nm}$ using a plastic cuvette filled with destilled water.

2. Sample liquid from the plastic flask is diluted with distilled water (1:10 to $1: 200)$ in a plastic tube to reach a suitable concentration. For experiments in series 1 and 2 the dilution is done using the volume scales on the plastic tubes, for experiments in series 3 and forward it is done using automatic pipettes. 
3. The diluted sample is poured into a cuvette of the same type as the calibration sample and placed in the spectrophotometer. Absorbance is noted.

4. If the absorbance is not within 0.2-1.2 absorbance units, steps 2 and 3 are repeated using a different dilution, which is expected to get an absorbance within these limits based on the previous results.

Measurement of glucose and acetate levels using Bioprofiler

1. Approximately $10 \mathrm{ml}$ sample is poured into a $10 \mathrm{ml}$ plastic tube and centrifuged at $2800 \mathrm{rpm}$ and $5{ }^{\circ} \mathrm{C}$, for 20 minutes.

2. Depending on the expected concentrations, the supernatant is diluted from 1:1 to 1:10 in an Eppendorf tube.

3. The Eppendorf tube is placed in a Bioprofiler machine, which gives concentrations for glucose and acetate in the sample.

4. If the concentrations are outside the range in which the Bioprofiler can make good measurements, steps 2 and 3 are repeated using a different dilution which is expected to give suitable concentrations for the Bioprofiler based on the previous results.

Measurement of glucose and acetate levels using enzymatic kits

1. Sample liquid from the plastic flask is drawn into a syringe.

2. A sterile filter (Minisart $\AA 0.20 \mu \mathrm{m}$ filter) is applied to the syringe and $1-2 \mathrm{ml}$ liquid is pressed through it into a $10 \mathrm{ml}$ plastic tube or a cryo tube which is to be used for enzymatic measurement of glucose levels.

3. Step 2 is repeated with a second tube which will be used for enzymatic measurement of acetate levels.

4. All tubes are placed in a freezer $\left(-18^{\circ} \mathrm{C}\right)$.

5. The enzymatic kits used are "D-Glucose" (Cat. No. 10716251 035) for determination of glucose concentrations and "Acetic acid" (Cat. No. 10148261 035) for determination of acetate concentrations (both by R-Biopharm AG). When the enzymatic kits are to be used, the tubes are taken from the freezer and thawed. The kits are used as per the instructions, with the exception that all volumes are halved in order to make the enzymatic kits last for longer.

Measurement of product activity

1. A separate sample is taken from the fermentor as per the description of general procedures.

2. The sample is marked and sent to a laboratory where product activity is measured. 